\title{
PARTICLE IDENTIFICATION AT AN ASYMMETRIC B FACTORY *
}

\author{
P. Coyle, G. Eigen, D. Hitlin, P. Oddone, \\ B. Ratcliff, N. Roe, J. VA'VRa and T. Ypsillantis \\ Stanford Linear Accelenator Center \\ Stanford University, Stanford, California, 94905
}

\begin{abstract}
Report of the Particle Identification Working Group of the Workshop on Physics and Detector Issues for a High Luminosity Asymmetric B Factory at SLAC, January-June 1990.
\end{abstract}

\section{DISCLAIMER}

\begin{abstract}
This report was prepared as an account of work sponsored by an agency of the United States Government. Neither the United States Cowernment wor any agency thereof, nor any of their employes, makes any warranty, express or implied, or assumes any legal liability of responsibility for the aceuracy, completeness, or usefulness of any information, apparatus, product, or process disclosed, or represents that jts use would not infringe privately owned rights. Reference herein to any specific commercial product, process, or service by trade name, trademark. manufacturer, or otherwise does not necessatily constituie or imply its endorsement, recom. mendation, of favoring by the Unired States Government os any agency thereos. The views and opinions of authors expressed herein do net necessarily stalc or reflect those of the United Stales Government of any agency thereof.
\end{abstract}

* Work supported by Department of Energy contract DE-AC03-76SF00515. 


\title{
REPORT OF THE PARTICLE IDENTIFICATION GROUP ${ }^{\star}$
}

\author{
P. Coyle, G. Eigen, D. Hitlin, P. Oddone,
}

B. Ratcliff, N. ROE, J. VA'vra and T. Ypsilantis

\section{INTRODUCTION}

D artjcle identification systems are an important component of any detector at a high-luminosity, asymmetric B Factory. In particular, excellent hadron identification is required to probe $C P$ violation in $B^{0}$ decays to $C P$ eigenstates. The particle identification systems discussed below also provide help in separating leptons from hadrons at low momenta.

We begin this chapter with a discussion of the physics motivation for providing particle identification, the inherent limitations due to interactions and decays in flight, and the requirements for hermiticity and angular coverage. A special feature of an asymmetric B Factory is the resulting asymmetry in the momentum distribution as a function of polar angle; this will also be quantified and discussed.

In the next section the three primary candidates, time-of-flight (TOF), energy loss $(d E / d x)$, and Cerenkov counters, both ring-imaging and threshold, will be briefiy described and evaluated. Following this, one of the candidates, a long-drift Cerenkov ring-imaging device, is described in detail to provide a reference design. Design considerations for a fast RICH are then described. A detailed discussion of aerogel threshold counter designs and associated R\&D conclude the chapter.

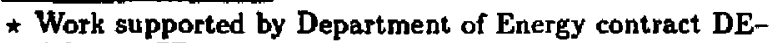
AC03-76SF00515. 
7 his section briefly summarizes the particle identification criteria which are dictated by the physics objectives of an asymmetric B Factory. We begin by discussing which particle species must be identified, and the momentum and angular distributions which are typical at an asymmetric machine. The limitations imposed by particle decays in flight are also considered. We will then consider a specific case, the "acid test" for any particle identification system: separating $B \rightarrow \pi \pi$ from $B \rightarrow K \pi^{1}$ These rare two-body decays set the maximum momentum range. Finally, we will consider the problem of flavor tagging $B$ 's by means of a kaon tag; this will give us a more typical momentum spectrum.

In the most general case there are five particle species which one would like to be able to distinguish at a B Factory: electrons, muons, pions, kaons and protons. Electrons and muons will be identified by the electromagnetic calorimeter and the muon system. There may be some difficulty separating muons from pions at low momenta, so it would be helpful if the particle identification system could provide some $\mu / \pi$ separation below about $600 \mathrm{MeV} / \mathrm{c}$. Fortunately, the low momentum range is where most devices under consideration work best and this complementarity is easily achieved. The remaining three particle species are all hadrons and require a device which can distinguish between them on the basis of mass. In general, the problem of $\pi / K$ separation is more difficult than that of $\pi / p$ or $K / p$ separation. Protons are also much less frequently produced in $B$ meson decays than are pions and kaons. We will therefore focus on $\pi / K$ separation as the most important criterion for particle ID performance.

The momentum distribution of pions produced in $B$ decays at an asymmetric machine with beam energies of 9 on $3.1 \mathrm{GeV}$ is shown in Figure 1(a); the corresponding distribution for kaons is shown in Figure 1(b).

The distributions peak toward low momenta; the average value for pions is $0.56 \mathrm{GeV} / \mathrm{c}$, and for kaons it is 


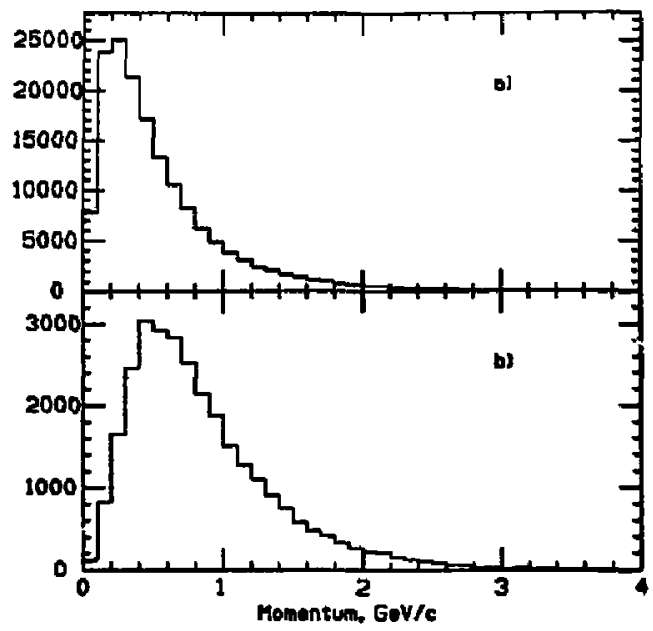

$0.85 \mathrm{GeV} / \mathrm{c}$. Eighty-six percent of all pions have momenta below $1.0 \mathrm{GeV} / \mathrm{c}$; the corresponding value for kaons is $7 C \%$.

The angular distribution for pions is shown in Figure 2 ; the angular distribution for kaons is very similar.

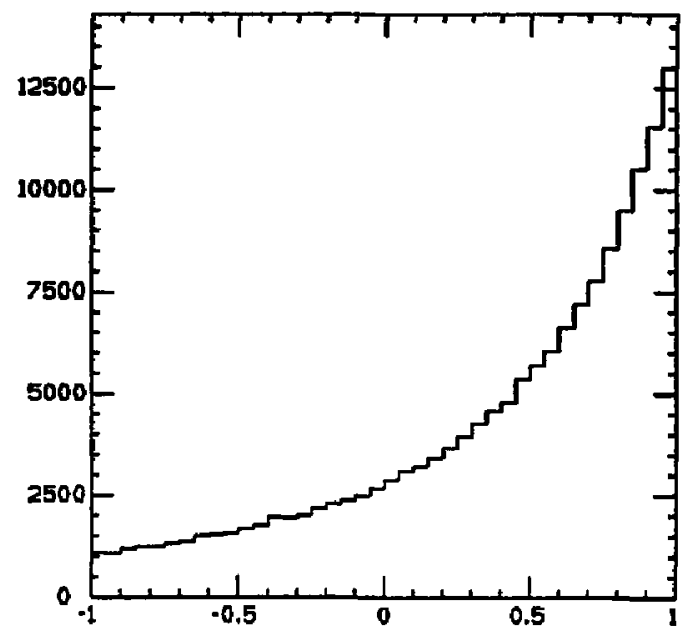

Figure 1. The momentum distribution for (a) pions and (b) kaons produced in $B$ meson decay's for beam energies of 9 on $3.1 \mathrm{GeV}$.
Figure 2. The angular distribution for pions produced in $B$ meson decays for beam energies of 9 on $3.1 \mathrm{GeV}$. 
Figure 3. The mean moment um distribution vs $\cos \theta$ for (a) pions and (b) kaons produced in $B$ meson decays.

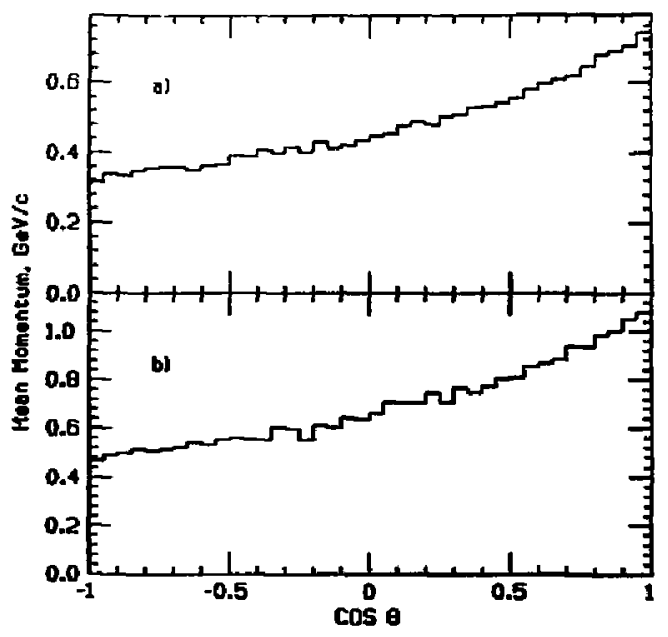

The distribution is asymmetric and forward-peaked due to the asymmetry in the beam energies. In Figure $3(a)$ the mean momentum as a function of $\cos \theta$ is shown for pions; Figure $3(\mathrm{~b})$ shows the same thing for kaons.

In the forward direction the mean momentum increases; for $\cos \theta=1$ it is more than twice that for $\cos \theta=-1$.

From Figures 2 and 3 we see that significantly more particles are produced in the forward direction, with significantly higher momenta. Therefore, particle identification will generally be both more important and more difficult in this region. For TOF and $d E / d z$ systems the increased path length for forward tracks relative to central tracks results in improved particle identification capability. This is especially important for the higher monentum tracks in this region.

Increased path length in the forward direction does have the disadvantage of increased particle decays. Decays in flight will prevent even the very best system from achieving perfect particle identification. The decay length distributions projected onto the plar.s perpendic- 


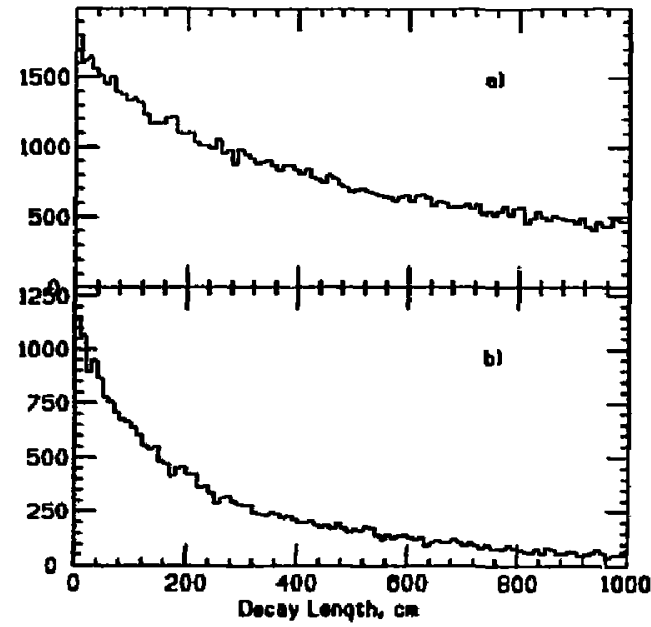

ular to the beam axis for pions and kaons produced in $B$ meson decays at an asymmetric machine with beam energies of 9 on $3.1 \mathrm{GeV} / \mathrm{c}$ are shown in Figures 4(a) and 4 (b).

Since $7.8 \%$ of pions and $24.6 \%$ of kaons vill decay inside a radius of $80 \mathrm{~cm}$, a particle identification device located outside this radius will misidentify a significant fraction of kaons unless the kink can be reconstructed. Since $63.5 \%$ of charged kaons decay into a muon, this will also present a serious background for the muon identification system. If we include an endcap particle identification system located at $z=150 \mathrm{~cm}$, the fraction of decays is reduced to $6.7 \%$ for pions and $21.6 \%$ for kaons. Using $d E / d x$ information from the drift chamber has the potential advantage over TOF or Cerenkov devices of reducing the misidentification probability due to decays in flight, although the precise level depends on the details of the $d E / d x$ algorithm used.

Next let us consider the specific example of separating $B \rightarrow \pi^{+} \pi^{-}$from $B \rightarrow K^{+} \pi^{-}$. These processes are expected to be rare, with calculated branching ratios on the order of $10^{-4}$ to $10^{-5}$. A measurement of $B \rightarrow \pi^{+} \pi^{-}$ can be used to extract $V_{u b}$ and, if the other $B$ is tagged, to measure $C P$ violation, while $B \rightarrow K^{+} \pi^{-}$proceeds
Figure 4. The decay lengths projected onto the $x, y$ plane for (a) pions and (b) kaons produced in $B$ meson decays. 
Figure 5. The momentum distribution for pions from the decay $B \rightarrow \pi^{+}$, for beam energies of 9 on $3.1 \mathrm{GeV}$.
Figure 6. The mean momentum vs, $\cos \theta$ for pions from the decay $B \rightarrow \pi^{+} \pi^{-}$, for beam energies of 9 on $3.1 \mathrm{GeV}$

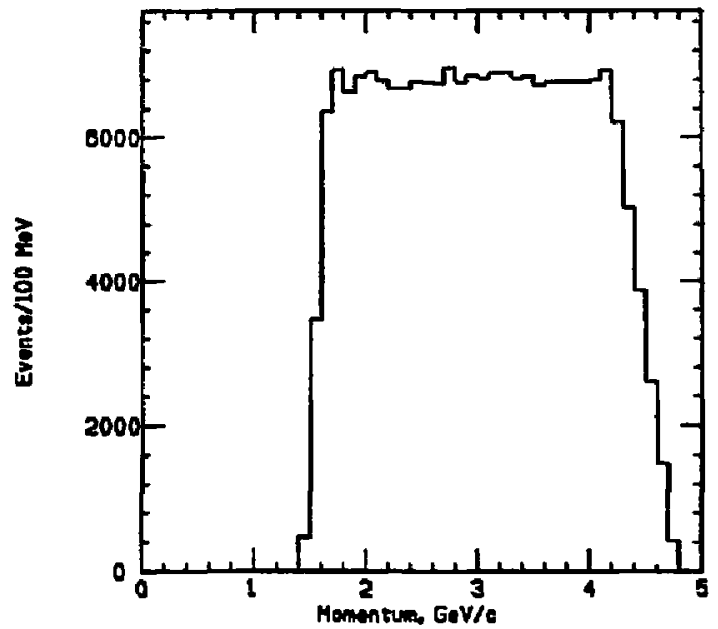

through a penguin diagram and is sensitive to the presence of heavy particles in the loop. These are important physics processes to measure, and they must be separated if the underlying physics is to be understood. For the case of a two-body decay into $\pi^{+} \pi^{-}$the momentum distribution of the pions is shown in Figure 5, and the average momentum as a function of $\cos \theta$ is shown in Figure 6.

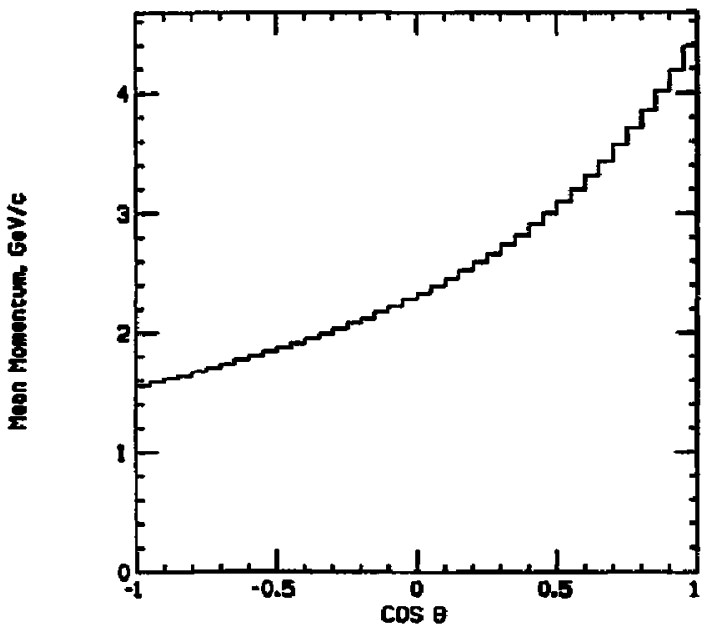


By comparison with the corresponding distributions for generic $\boldsymbol{B}$ meson decays (Figures 1 and 3) we see that the pions from the two-body decay have much higher momentum, and they will therefore pose a much bigger challenge to the particle identification system. Let us first consider whether these two processes could be kinematically separated. For a resolution on transverse momentum given by $\sigma_{p_{t}} / p_{t}^{2}=0.23$, the reconstructed invariant mass of the $\pi \pi$ system has $\sigma_{M}=22 \mathrm{MeV} / \mathrm{c}^{2}$. If the $K \pi$ final state is reconstructed assuming the pion mass for both particles, the mean of the resulting invariant mass distribution is $44 \mathrm{MeV}$ below the nominal $B$ meson mass and has $\sigma_{M}=24 \mathrm{MeV} / \mathrm{c}^{2}$; see Figure 7.

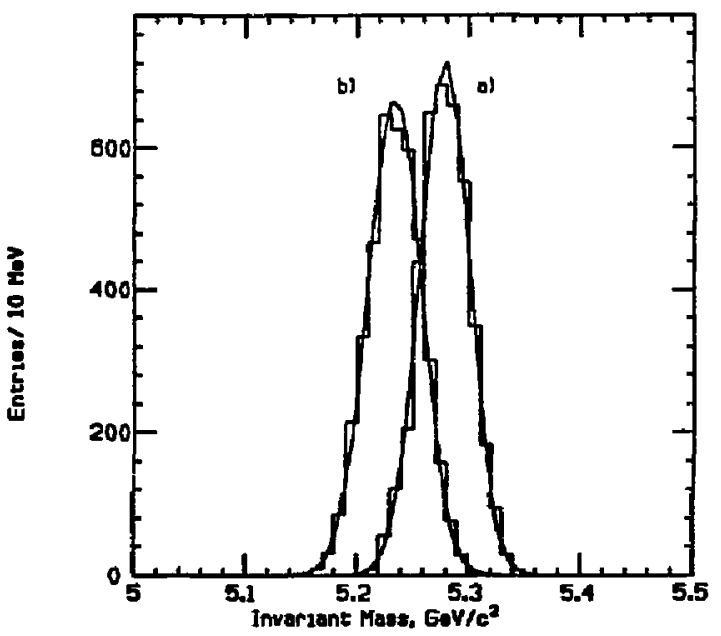

It is clearly difficult to separate these processes kinematically. In principle one could impose a cui on the invariant mass requiring $M_{\pi \pi}>5.290 \mathrm{GeV} / \mathrm{c}^{2}$, for example, which would reject $99 \%$ of the $K \pi$ events while retaining $30 \%$ of the $\pi \pi$ events. However, one loses efficiency in a channel which is rare to begin with, and furthermore one is forced to rely heavily on Monte Carlo

* The mass resolution can be improved by an order of magnitude if one boosts back to the $\mathbf{T}(\mathbf{4 S})$ rest frame and uses the beam energy constraint; however, in order to perform the boost one must know the particle masses.
Figure 7. The invariant mass distributions for (a) $B=$ $\pi^{+} \pi^{-}$and (b) $B \rightarrow K^{\circ}$. The latter was calculated assuming both particles were pions. 
simulation of the tails of the invariant mass distribution. For these rare decays it is desirable to have a particle identification system capable of performing $K \pi$ separation for momenta up to about $4.5 \mathrm{GeV} / \mathrm{c}$. For this purpose it is likely that only a Cerenkov ring-imaging system will suffice.

Finally, let us consider the less stringent case of $\pi / K$ separation for the purpose of kaon tagging. This technique, in which the sign of the kaon is used to tag whether a $B$ or a $\vec{B}$ decayed, is important as a means of enhancing the tagged sample for $C P$ violation studies. The semi-leptonic decays are also useful for tagging, but have a smafler total branching fraction. Also, cuts must be applied to reduce the contribution from charmed semileptonic decays. Kaon tagging relies on the fact that in the cascade $b \rightarrow c \rightarrow s, a b$ quark will produce a $K^{+}$ and a $\bar{b}$ quark a $K^{-}$. There is some wrong-sign contamination from Cabibbo-suppressed decays, which can be reduced by rejecting events with extra $K_{s}^{0}$ 's or multiple charged kaons.?

To evaluate these effects we have considered a simplified tagging scheme which requires one charged kaon and no extra $K_{S}^{0}$ 's." Only those $K_{S}^{0}$ 's which decayed to charged pions were used as a veto. All particles were required to have $|\cos \theta|<0.95$ and $p_{t}>40 \mathrm{MeV} / \mathrm{c}$. With no decays and $100 \%$ particle ID, $38 \%$ of all events were tagged, with $94 \%$ of the tags being correct. If we turn on $K$ decays in flight, $31 \%$ of events are tagged with $91 \%$ being correct. The increased fraction of wrong-sign tags is due to feed-down from events with multiple charged kaons. The effect. of decays in flight is therefore to reduce the tagging efficiency by $18 \%$ while increasing the fraction of wrong-sign tags by $50 \%$.

Next we will include the effect of particle identifica. tion. The momentum distribution for tagging kaons is shown in Figure 8. It is slightly harder t:'an the momentum distribution for all kaons; the mean is $0.92 \mathrm{GeV} / \mathrm{c}$.

* The $B$ and $D$ decays were simulated using the ASLUND decay table described in SLAC-353 
We will consider three possible particle identification systems; TOF with a resolution of $75 \mathrm{ps} ; d E / d x$ with a resolution of $7.5 \%$; and a liquid CRID with the performance described in Section 1.4 below. Each device is assumed to be $100 \%$ efficient for $|\cos \theta|<0.95$. An identified kaon must be within $3 \sigma$ of the value expected for a kaon and at least $2 \sigma$ away from that expected for a pion. The resultant efficiencies for a tagging kaon are $90 \%$ for TOF, $57 \%$ for $d E / d x$, and $99 \%$ for CRID. The $d E / d x$ efficiency is low because the cross-over region between $\pi$ 's and $K$ 's is around $1 \mathrm{GeV} / \mathrm{c}$, where $\pi / K$ separation is poor but many tagging $K$ 's are found. The corresponding misidentification probability for $\pi$ 's is $0.3 \%$ for TOF, $0.9 \%$ for $d E / d x$ and $0.04 \%$ for CRID. Given that there are approximately $6.3 \pi$ 's per $h^{\prime}$ in a typical $B$ decay, the additional fraction of wrong-sign tags due to misidentified $\pi$ 's would be $2 \%$ for TOF, $6.4 \%$ for $d E / d x$, and $0.3 \%$ for CRID. (This takes into account the fact that events with two or more charged K's are not counted as tags.) Relaxing the particle ID requirements would improve the efficiency, but at the cost of increasing wrong-sign tags due to misidentified pions.

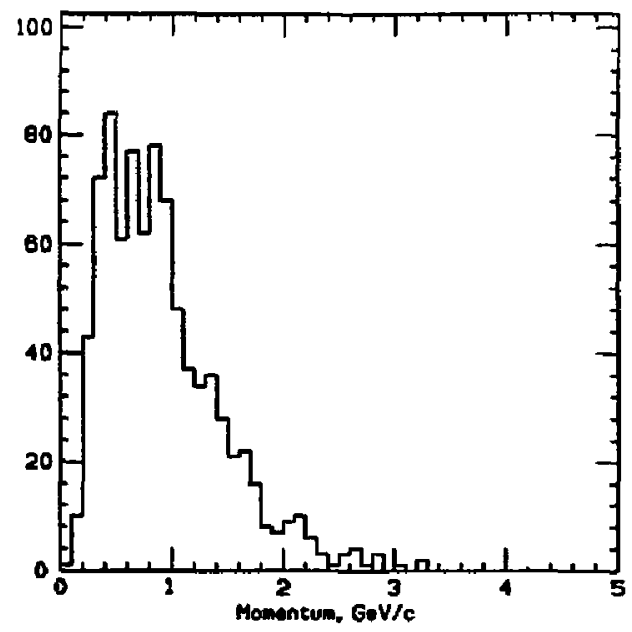

In conclusion, particle identification efficiency must be good in both the medium momentum range, for effi-
Figure 8. The momentum distribution for tagging kaons. 
ciency in kaon tagging, and at the high end of the momentum range, to separate rare two-body decays. A very good TOF system is adequate for the former but totally inadequate for the latter. The hypothesized $d E / d x$ system has a disappointing performance for both, because the $\pi / K$ crossover makes it inefficient for kaon tagging while the separation at high momenta is not very gcod. A CRID, which has the disadvantage of being the most expensive and complex device to build, gives the best performance in both cases. Other devices, such as threshold counters, or better quality $d E / d x$, remain to be evaluated in this model.

\section{Technological Choices for Particle Iden- TIFICATION}

7 his section briefly summarizes the basic properties of the candidate technological choices available for particle identification at the B Factory. There appear to be only three candidates that could cover a fair portion of the momentum range with good performance; (1) time-of-flight (TOF); (2) ionization loss in gaseous detectors $(d E / d x)$; and (3) Cerenkov counters. Moreover, the choice of technology is constrained not only by physics requirements, but by the machine environment and the properties of the remainder of the detector. Thus, a number of properties of each technology, in addition to specific performance, must be considered, including (1) mass; (2) size/space requirements; (3) decay/interaction limits to performance; (4) performance at high rates; (5) triggering considerations; (6) coverage and hermiticity; ( 7 ) construction and operating costs.

The TOF technique is clearly useful and well understood at low momentum, but above about $1.5 \mathrm{GeV} / \mathrm{c}$ requires either that the resolution reach unprecedented precision or that the flight path (and thus the whole detector) be unacceptably large. Modest quality $d E / d x$ in a central tracker sufficient to provide good hadronic separation in the $1 / \beta^{2}$ region and to help in low momentum $e / \pi$ discrimination is likely to exist in any modern tracking device essentially for "free." However, high 
momentum $\pi / K$ separation (above about $1.5 \mathrm{GeV} / \mathrm{c}$ ) requires working in the relativistic rise region of the energy loss curves, and leads to a requirement for many samples (and therefore a large chamber), and also suggests a heavier gas (perhaps pressurized) than would be desired for the best tracking resolution. In addition, the existence of a "cross-over" between the $1 / \beta^{2}$ and relativistic rise regions requires that it must be combined with another technology (such as TOF) if complete momentum coverage is to be attained. Such a combined system could perform the particle ID task adequately and cioes have a number of attractive features. The central chamber is much larger and more massive than a tracking only device, but it does "double duty." Track matching is simple and the device is very uniform geometrically. Moreover, 'le large dip angles (with the highest momeritum tracks) also have the largest sample lengths and thus the best resolution, matching the requirements of the asymmetric design. The particle ID in such a device starts at the snjaliest possible radius and thus should be the least sensitive to misidentification due to particle decay, Finally, the separation attainable in the relativistic rise region is nearly independent of monientum from $2 \mathrm{GeV} / \mathrm{c}$ to well above the maximum momentum seen at the B Factory.

The third (and leading) contender for hadronic identification at the B Factory is the Cerenkov technique. As will be discussed below, either threshold or ring imaging techniques could provide adequate performance, in principle, provided that the efficiency for Cerenkov photon detertion can be made sufficiently high. The primary conceptual advantages over $d E / d x$ are that the devices (1) are generally rather thin radially (typically around $20 \mathrm{~cm}$ ), leading to a smaller overall detector size and less expensive calorinetiy: (2) are fully modular and separable from the tracking, allowing the optimization of the tracking independent of particle ID concerns; (3) have excellent nominal separations (especially for the ring imaging devices) over the phase space of the B Factory; (4) are quite robust against degradation in performance, in contrast to the requirements on $d E / d x$ in the relativistic rise regıon. Conversely, the Cerenkov devices 
Figure 9. Maximum momentum at which $\pi / K$ separation exceeds $3 \sigma$ as a function of the TOF counter radius and resolution.

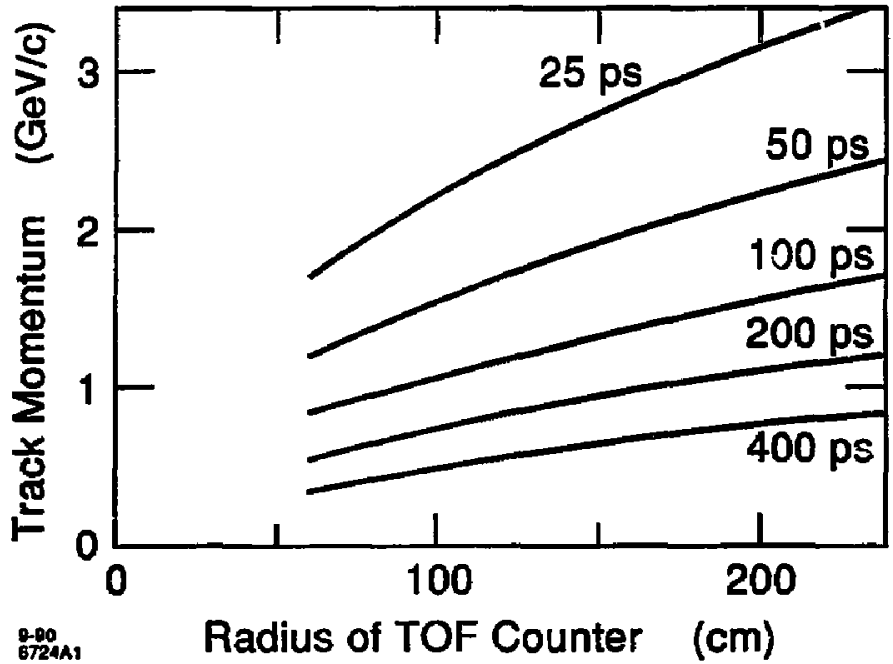

are probably even more challenging to construct and operate, may add more mass in front of the calorimeter, and are probably more difficul: to build in a fully hermetic configuration.

In the following subsections, the general pr.sperties of each technology are discussed in somewhat more detail. The design realizations for a B Factory are presented in the subsequent sections.

\subsection{The Time-of-Flight Technique}

The time-of-flight (TOF) technique is well-known; this review will therefore be brief. ${ }^{3}$ It has been extensively applied in earlier experiments to identify low momentum particles. In $e^{+} e^{-}$collider experiments, a typical system consists of a cylindrical bank of scintillators positioned so as to maximize the flight path, e.g., surrounding the central tracker just inside the calorimeter. The time of passage of the particle through these counters stops a clock which was started by the beam crossing signal from the mac hine giving the time-of-flight ( $\mathrm{T}$ ). Photomultiplier tubes are generally included on each end so as to give two independent measures of the stop time, 
and a $\sqrt{2}$ improvement in resolution. The length $(L)$ of the particle's trajectory is calculated with high accuracy from measurements in the tracking chamber. The velocity $(\beta)$ of the particle can be calculated as $\beta=L / c T$.

The TOF technique using scintillation counters is simple, well understood, and robust. The problem for the B Factory is that it is not effective at sufficiently high momentum. The fractional error on a particle of mass $(M)$ and velocity $\beta$ scales like $\gamma^{2}$ [i.e., like $1 /\left(1-\beta^{2}\right)$ ] for $\beta \approx 1$. Thus, for fixed time resolution, the path length required to attain a given separation between particle species is a rapidly increasing function of momentum. ${ }^{4}$

Figure 9 gives the upper limit of the momentum range over which $3 \sigma \pi / K$ separation is possible as a function of the radius of the TOF system. The situation is shown for the shortest path length particles in the usual solenoidal geometry, and assumes a $1.0 \mathrm{~T}$ field, although the path lengths for fast particles are nearly independent of the field. Since the path length $L$ increases approximately like $1 / \cos \theta_{d}$, where $\theta_{d}$ is the dip angle, an asymmetric collider has a "natural" path length enhancement in that the highest momentum particles which tend to go forward get the largest path lengths. On the other hand, a large magnetic field will cause the softest particles to curl and be lost from TOF identification.

The best resolutions which can be attained with "conventional" scintillation counters in a collider environment are optimistically around $\sigma=100 \mathrm{ps} .{ }^{5}$ This limits the $\pi / K$ separation to momentum below $1.1 \mathrm{GeV} / \mathrm{c}$ for a TOF counter with a $1 \mathrm{~m}$ radius. More typically, resolutions obtained in experiments are around 110 to $150 \mathrm{ps}^{6}{ }^{6}$ In principle, other technological approaches to fast timing, such as spark gap counters, can attain substantially better resolution. ${ }^{3}$ However, they are difficult to work with, and have yet to be used successfully in a large $4 \pi$ acceptance detector. If a TOF scintillation counter were combined with $d E / d x$ in a tracking chamber, the radius of the counter would need to be sufficient to cover the $\pi / K$ crossover region in ionization loss which extends from about 800 to $1600 \mathrm{MeV} / \mathrm{c}$. This leads to a minimum 
radius counter of about $2 \mathrm{~m}$, assuming 100 ps resolution. Though reasonably consistent with the radius needed for a good $d E / d x$ device operating in the relativistic rise region, it leads to a large, expensive calorimeter, and the large path lengths before TOF ID will lead to misidentification problems for particles which decay.

There are a few other issues of B Factory TOF counter design which should be briefly mentioned. First, the resolution obtainable in a scintillation TOF counter depends on the length of the counter, mostly due to photon absorption inside the scintillator. A typical effective absorption length is about $2 \mathrm{~m}$, which limits the practical counter length to be around 4 to $5 \mathrm{~m}$. With a device at a radjus of $1.6 \mathrm{~m}, \mathrm{a} 4 \mathrm{~m}$ long counter could cover only down to $39^{\circ}$ in the lab, necessitating end cap counters to cover the forward/backward regions. Second, the variation in flight and collection time combined in a long counter is of order 15 to $20 \mathrm{~ns}$. So when the design machine bunch spacing is of this order or less, a simple, single hit indication of which bunch to associate with the track is no longer available. Third, the start time of the TOF system is determined synchronously by the beam crossing, but the event can occur at any time within the colliding beam overlap time. Thus, the longitudinal size of the B Factory beams provides a "hard" limit to the resolution attainable in the system. For example, if each beam is $1 \mathrm{~cm}$ long, the contribution to the resolution from the start time alone is about 50 ps. In principle, this resolution could be improved stochastically by averaging over the tracks in the event. Fourth, a TOF counter system is massive, placing about $15 \%$ of a radiation length of material in front of the calorimeter. On the other hand, the good pulse height resolution necessary to attain the best timing resolution also makes it possible to measure the energy deposited, so the low energy performance of the calorimeter should not be significantly affected. 


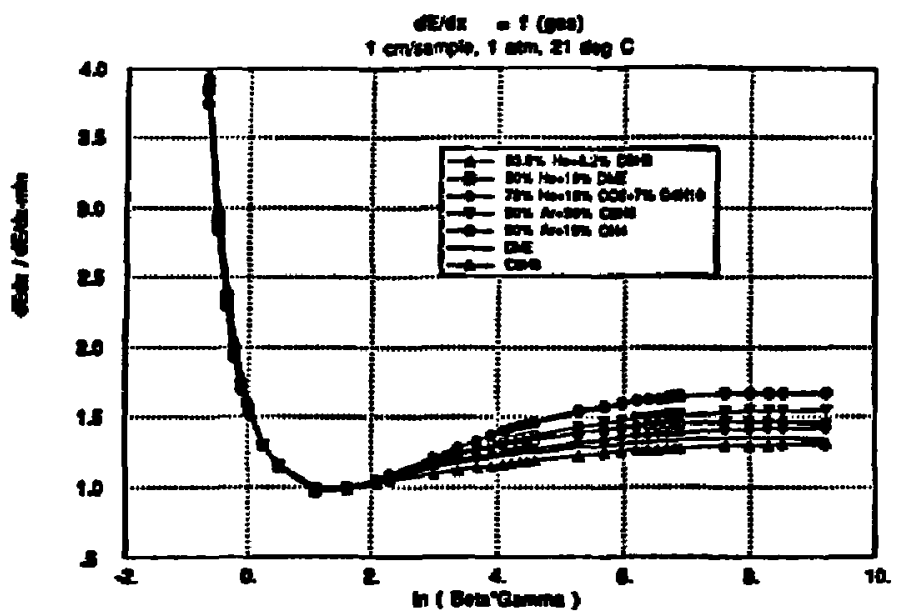

Figure 10, Most probable energy loss $(d E / d z)$ versus $\ln (\hat{\beta} \gamma)$ for several di : " gas mixtures : 1 atm pressure and $21^{\circ} \mathrm{C}$.

\subsection{The $d E / d x$ Technique}

In recent years, many of the large-scale detectors which have been built have incorporated a measurement of particle energy loss $(d E / d x)$ in a gas as a central element of their design. The acronyms of some of the detectors that have succesfully implemented this technique are TPC, EPI, JADE, CLEO, ARGUS, OPAL, ALEPH, DELPHI, and MARK II; and this list is far from exhaustive. ${ }^{7}$ The energy loss of a particle in a gas is a function of the properties of the gas and the velocity of the particle, as shown in Figure 10. It is conventional to divide this curve into a low velocity region (the $1 / \beta^{2}$ region; the minimum ionizing region of around $\ln (\beta \gamma)=1$ ]; the relativistic rise region; and the relativistic plateau at high velocity. Since the curve of energy loss versus velocity for a particular gas depends only on particle velocity and gas composition, when coupled with a momentum measurement in the magnet, it determines particle mass.

The identification is not always unique. There are ambiguities in the so called "cross-over" regions, where two particles of different mass but the same momentum produce an equal energy loss (see, e.g., Figure 12 below). In these regions, another method of particle identification is required. As mentioned above, a major advan- 
tage of using $d E / d x$ for particle identification is that the detector that measures the energy loss can simultaneously measure the track of the particle, saving space and greatly reducing confusion in associating the energy loss measurements with tracks.

To achieve good hadron-hadron identification in the relativistic rise region, the device must be very large ( $\geq 150 \mathrm{~cm}$ ); have many samples; operate at high gas pressure; or, most likely, all of the above. There is no "magic gas" choice which can alter these basic requirements. This can be shown either empirically, ${ }^{8}$ or using a Monte Carlo calculation, ${ }^{9}$ or using a phenomenological calculation. ${ }^{10}$ This results from the fact that any single energy loss measurement on a thin gaseous sample has large Landau fluctuations, and at the same time different particles do not yield very different energy losses, with the exception of the nonrelativistic region. In principle, cluster counting could improve $d E / d x$ performance, but there is, as yet, no successful implementation of this technique. Examples of experiments with good $d E / d x$ performance are TPC, ALEPH, and OPAL, and they provide some examples of different optimizations. TPC has $1834 \mathrm{~mm}$ samples over a path length of $73 \mathrm{~cm}$ at a gas pressure of 8.5 atm.; ALEPH has $3404 \mathrm{~mm}$ samples over a path length of $136 \mathrm{~cm}$ at a gas pressure of 1 atm.; and OPAL has $1201.3 \mathrm{~cm}$ samples over a path length of $160 \mathrm{~cm}$ at a gas pressure of $4.0 \mathrm{~atm}$.

However, $d E / d x$ measurement can still be useful for smaller devices, only $1 \mathrm{~m}$ long, with 60-100 samples operating at 1 atm. In this domain one can perform hadron-electron identification into the few $\mathrm{GeV} / \mathrm{c}$ range, and hadron-hadron identification in the nonrelativistic region (below $\sim 1 \mathrm{GeV} / \mathrm{c}$ ). If the nonrelativistic region is the only region of interest, it is advantageous either to use heavy gases such as propane or DME, or to increase the gas pressure, in order to improve the resolution. In the relativistic region, this trick is not as effective because one also reduces the relative height of the relativistic plateau with respect to the minimum energy loss, thus negating much of the advantage gained from smaller resolution. An example of a detector optimized 
for the the lower velocity region is ARGUS, which uses propane gas.

In understanding the relationship of parameter choices made in various designs, it is helpful to use the following approximate empirical scaling relationships ${ }^{11}$ for the resolution on $d E / d x$ :

$$
\begin{aligned}
d E / d x \text { Resolution } & \sim \text { (Pressure }^{-0.32} \\
& \sim \text { (Sample Thickness }^{-0.32} \\
& \sim \text { (Total Number of Samples) }^{-0.43}
\end{aligned}
$$

The dependence of the resolution on gas composition can be expressed approximately by the following formula (see the notes following the table below for definitions of variables):

$$
d E / d x \text { Resolution } \sim\left(\frac{\alpha t}{I \beta^{2}}\right)^{-0.32}
$$

In the relativistic region, the size of the relativistic plateau can be affected by both the gas pressure and the gas density. Figure 10 shows that the denser gases have a smaller relativistic plateau. Similarly, the pressure dependence of the relativistic rise can be parametrized approximately as follows (see the notes following the table below for definitions of the variables):

$$
\text { Relativistic Rise }\left(E_{\max } / E_{\min }\right) \sim 1.6 \times \text { (Pressure }^{-0.09}
$$

The incorpos -tion of $d E / d x$ measurement in a shatber requires that a number of tight constraints on the tracking device design be enforced. Although these are now generally understood, they should not be underestimated. Usually they degrade the $d E / d x$ performance achieved in practice. Therefore, one should assume that a "minimal $d E / d x$ system" must be designed with at least $4 \sigma$ of theoretical particle separation. A few areas of concern follow:

1. All mechanical tolerances must be carefully controlled and their stability ensured.

2. The high voltage power supply must be kept stable. 
3. The total gas gain should be limited to $\sim 5 \times 10^{4}$ if a jet cell with charge division is used, and preferably to a much lower value in a TPC. This is necessary to reduce the track angle dependent corrections due to the wire gain saturation effects.

4. Wire-to-wire cross-talk in jet cells should be compensated by hardware.

5. Pulse digitization techniques (e.g., flash-ADC's) should be used to resolve close tracks in the jet cells. For this reason, a chamber design with small cells may be easier to use.

6. A number of background problems and associated space charge effects, efficiency gating, etc., can distort a track and possibly affect the $d E / d x$ sampling.

7. A $d E / d x$ measurement must deal with many corrections, e.g., because of the gain variation due to temperature, barometric pressure, and gas composition; or other effects due to wire staggering, electrostatic and gravitational deflections, space charge effects, etc.

8. Many details of the electronic design can also affect the performance. Due consideration must be give to electronic noise, stability, base line shifts, cross talk, dynamic range, chamber termination, etc.

\section{$B$ Factory Design}

From a tracking and calorimetric perspective, the optimized tracking device for a B Factory should be modest in size and low in density. Here, we will consider the $d E / d x$ performance of such a chamber with $60(1 \mathrm{~cm})$ samples operating at 1 atm. pressure. The performance of helium-based gases will be compared with the performance of heavy gases like propane or DME. The calculations are done using the phenomenological method. ${ }^{10} \mathrm{~A}$ similar method was used in the low momentum regime for the Tau-Charm Workshop. ${ }^{10}$ Here, we extend the results up to $6 \mathrm{GeV} / \mathrm{c}$.

Table 1 shows a brief summary of parameters for a variety of gases, including their expected $d E / d x$ resolution for a single $1 \mathrm{~cm}$ sample, and the size of the 


\begin{tabular}{|l|c|c|c|c|c|}
\hline Gas & $\begin{array}{c}\rho\left(\text { at } 21^{\circ} \mathrm{C} \text { and } 1 \mathrm{~atm} .\right) \\
(\mathrm{g} / \mathrm{cm} 3)\end{array}$ & $I(\mathrm{ev})$ & $\frac{\alpha t}{I}$ & $\frac{E_{\max }}{E_{\min }}$ & $\begin{array}{c}\text { FWHM } \\
\left(E^{-1}\right)\end{array}$ \\
\hline $\mathrm{C}_{3} \mathrm{H}_{8}$ & $1.88 \times 10^{-3}$ & 50.3 & 3.39 & 1.30 & 0.55 \\
\hline $90 \% \mathrm{Ar}+10 \% \mathrm{CH}_{4}$ & $1.57 \times 10^{-3}$ & 191.2 & 0.58 & 1.67 & 0.96 \\
\hline $50 \% \mathrm{Ar}+50 \% \mathrm{C}_{2} \mathrm{H}_{6}$ & $1.46 \times 10^{-3}$ & 129.1 & 0.89 & 1.54 & 0.84 \\
\hline $\mathrm{DME}$ & $1.89 \times 10^{-3}$ & 59.8 & 2.74 & 1.35 & 0.59 \\
\hline $90 \% \mathrm{He}+10 \% \mathrm{DME}$ & $3.39 \times 10^{-4}$ & 43.8 & 0.64 & 1.47 & 0.94 \\
\hline $78 \% \mathrm{He}+15 \% \mathrm{CO}_{2}+7 \% \mathrm{C}_{4} \mathrm{H}_{10}$ & $5.76 \times 10^{-4}$ & 50.0 & 0.93 & 1.43 & 0.83 \\
\hline $93.8 \% \mathrm{He}+6.2 \% \mathrm{C}_{3} \mathrm{H}_{8}[13]$ & $2.73 \times 10^{-4}$ & 42.5 & 0.53 & 1.48 & 0.99 \\
\hline
\end{tabular}

relativistic plateau as calculated using the phenomenological method. ${ }^{10}$ The $d E / d x$ value corresponds to the most probable energy loss. The resolution is scaled to one sample after performing a "truncated mean" operation in which about $40-50 \%$ of the highest samples are thrown out to get rid of the Landau tail. $E_{\operatorname{ma}} / E_{\min }=$ $\left(d E / d x_{\max }\right) /\left(d E / d x_{\min }\right)$ is the ratio of the relativistic plateau to the minimum energy loss; FWHM $\left(E^{-1}\right)$ is the full width at half maximum of the resolution for a single $1 \mathrm{~cm}$ sample divided by the most probable energy loss; $I$ is the mean ionization potential of the gas mixture; $p$ is the gas density; $t$ is the sample thickness $(1 \mathrm{~cm})$; and $\alpha t=0.153(Z / A)$ pt $\left(\mathrm{MeV} /\left(\mathrm{gm} / \mathrm{cm}^{2}\right)\right)$.

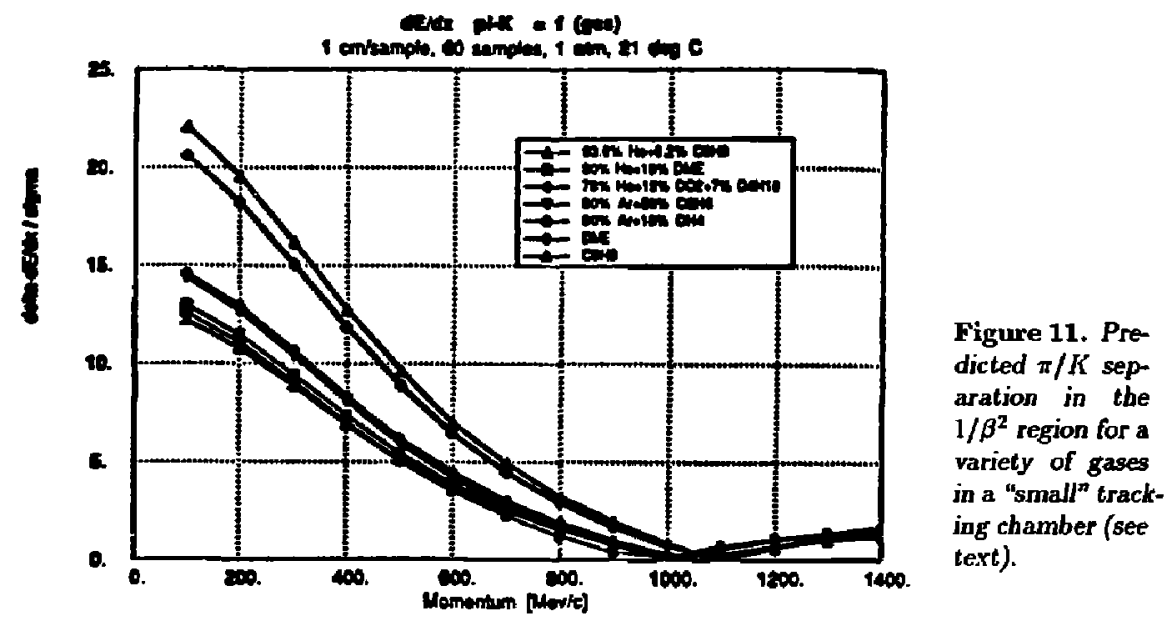

Table 1. Parameters of various which are relevant to $d E / d x$ measurements. 
Figure 12. Predicted hadronhadron separation performance as a function of momentum for a "small" tracking chamber (see text).

Figure 13. Predicted hadronelectron separation performance as a function of momentum for a "small" tracking chamber (see text).

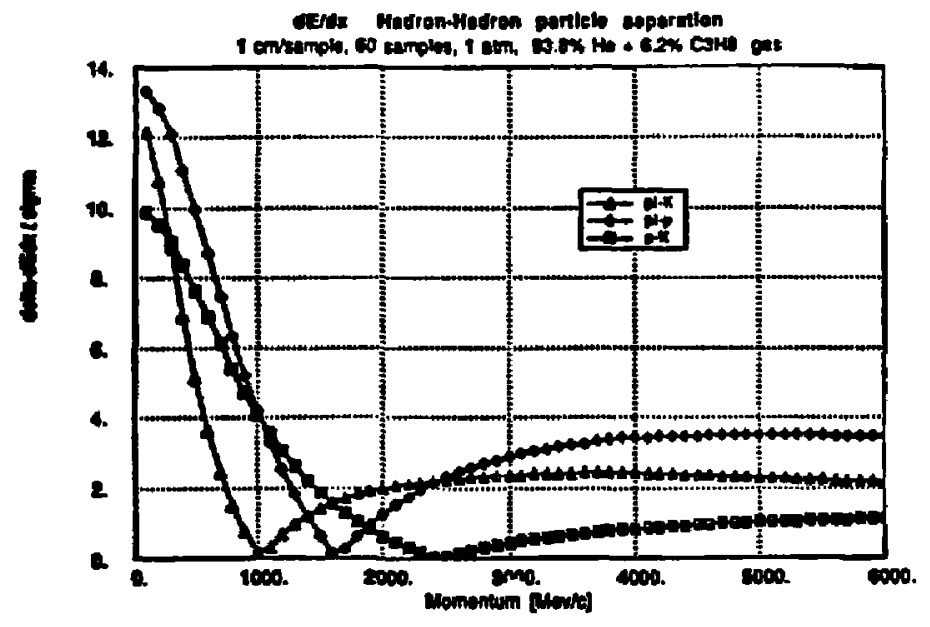

Figure 11 shows the predicted performance of this system for $\pi / K$ separation in the $1 / \beta^{2}$ region as a function of different gases. Dense gases clearly perform better. Above $1 \mathrm{GeV} / \mathrm{c}, \pi / K$ separation is hopeless for such a small $d E / d x$ system with any gas. Perhaps there is some modest $\pi / p$ separation, as can be seen in Figure 12 .

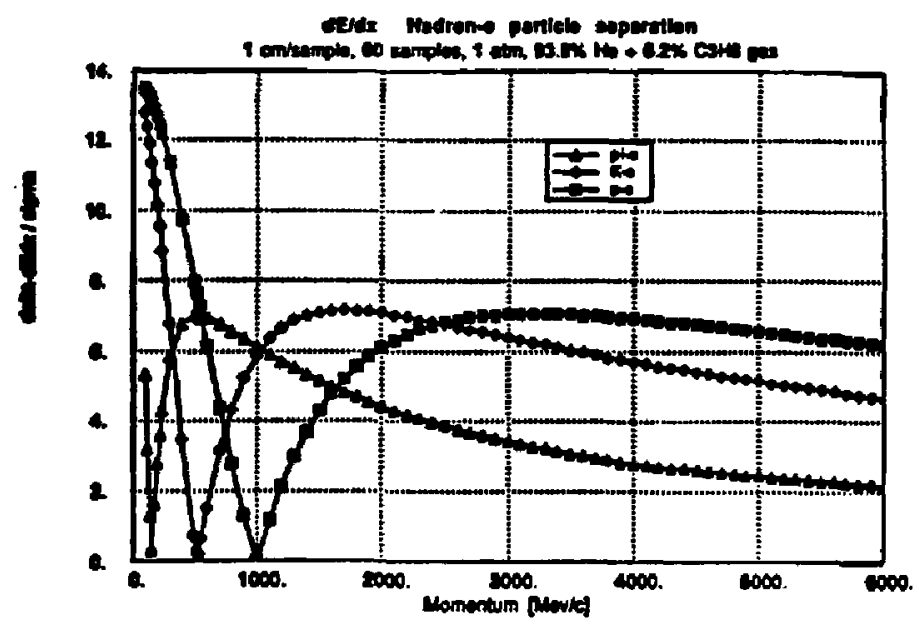


However, as shown in Figure 13, hadron-electron identification is quite satisfactory over the entire momentum region (except for the cross-over regions), even with a relatively small $d E / d x$ system.

One may ask how to change the tracking design in a minimal way so that the $d E / d x$ system is capable of doing $\pi / K$ particle identification above $2 \mathrm{GeV} / c$. There is no unique prescription for such a chamber, but it must have the general features discussed above. Here, we consider a device with 200 samples each $1 \mathrm{~cm}$ long, running at 2 atm. with helium-based gas. Figures 14 and 15 show the results.

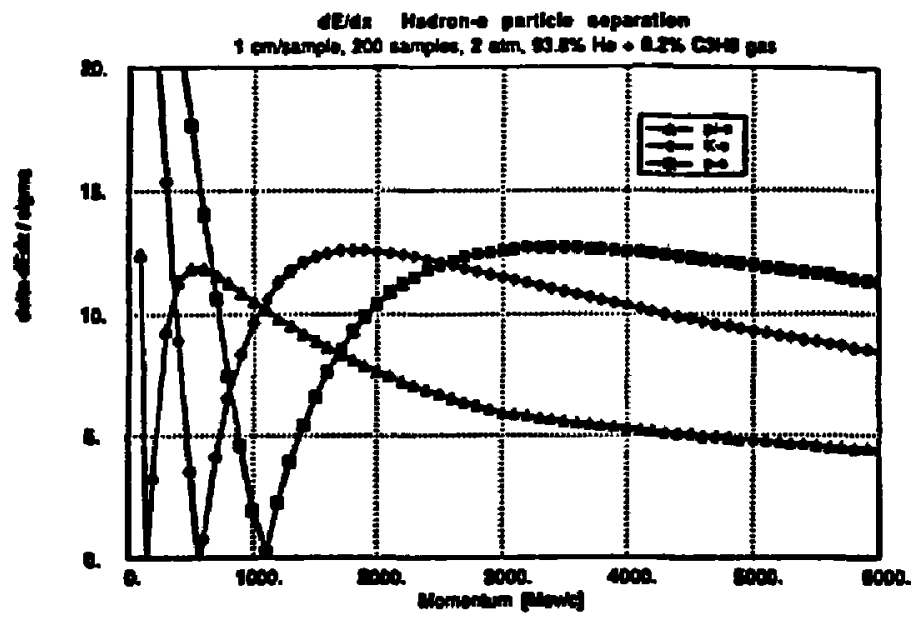

Figure 14. Predicted hadronelectron separation performance as a function of momentum for a $200 \mathrm{~cm} \mathrm{~B} \mathrm{Fac-}$ tory chamber design (see text).

Clearly, while hadron-electron, $\pi / p$ and $\pi / K$ separations are acceptable, $p / K$ separation in the region above $2 \mathrm{GeV} / \mathrm{c}$ is not. The cross-over regions are very large and difficult to cover with other techniques. Moveover, such a large device would increase substantially the size (and the cost) of the calorimetry, and the increased mass in the pressure vessel walls would decrease the low momentum tracking resolution and the low energy photon detection efficiency and resolution. 
Figure 15. Predicted hadronhadron separation performance as a function of momentum for a $200 \mathrm{~cm}$ $B$ Factory chamber design (see text).

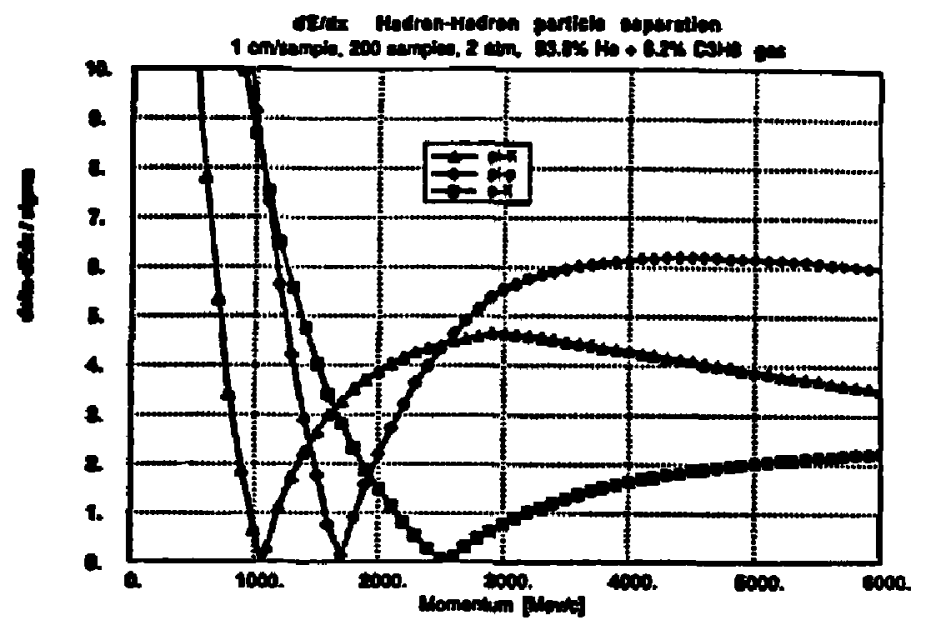

3.3. The Cerenkov Technigue

\section{Introduction}

The detection of Ċerenkov radiation has been an invaluable tool for measurement of charged particle velocity since its first quantitative measurements in 1934 by Cerenkov and subsequent theoretical explanation by Tamm and Frank in 1937. When combined with a momentum measurement of the charged track in a magnetic field, excellent particle identification discrimination is achievable. In this section we first briefly discuss the theory of Cerenkov radiation and collect together some equations relevant for detector design. The varjous choices and options available to the Cerenkov designer are then discussed and technologies relevant to B Factory designs are considered. A number of excellent reviews cover most of this material in detail. ${ }^{12,13}$

\section{Some Cerenkov Theory}

The passage of a charged particle of velocity $v=\beta$, through a dielectric medium of refractive index $n$ will induce transient dipoles within the atoms of the medium. When the particle velocity is greater than the velocity of light in the medium, the radiation emitted from these 
dipoles adds coherently to give a detectable signal. The Cerenkov radiation is confined to a cone of angle $2 \theta_{c}$ about the particle direction, where the cone angle is given by,

$$
\cos \theta_{c}=1 / \beta n \text {. }
$$

The emission angle is random in the azimuthal direction. As $\cos \theta_{c}$ can never be greater than one. Cerenkov radiation can only be emitted if

$$
\beta>\frac{1}{n}
$$

The Cerenkov radiation has an energy dependence $(E)$ and is detectable between two energies $\left(E_{L}\right.$ and $\left.E_{H}\right)$. It can be shown that the number of observed photons $N_{D}$ per unit radiator length (L) is given by

$$
\frac{d N_{D}}{d L}=370 \cdot Z^{2} \int_{E_{L}}^{E_{H}}\left(1-\frac{1}{(\beta n(E))^{2}}\right) \epsilon(E) d E
$$

where $Z$ is the charge of the particle; the energy unit is electron volts; and the length unit is centimeters (cm). The efficiency factor $\epsilon(E)$ parametrizes the overall detection efficiency and may include losses due to window and radiator transmissions, reflection at surfaces, photocathode efficiencies of the detector, etc. The integration bandwidth is essentially the range over which $\epsilon$ is finite and $\beta n(E)>1$.

Under the approximation that the medium is nondispersive, it is convertional to clump the efficiency factor with the numerical constant to form a new constant $N_{0}$ given by

$$
N_{0}=370 \int_{E_{L}}^{E_{H}} \epsilon(E) d E
$$

Thus, for a charged particle of unit charge the number of detectable photoelectrons is

$$
N_{\text {p.e. }}=N_{0} L\left(1-\frac{1}{(\beta n)^{2}}\right)=N_{0} L \sin ^{2} \theta_{c}
$$


$N_{0}$ is the figure of merit for a detector and allows detector performances to be compared independently of the radiator length and refractive index. Typical $N_{0}$ 's for useful detectors range from 30 to $200 \mathrm{~cm}^{-1}$. For example, a photomultiplier tube operating in the visible $\left(E_{L}=2 \mathrm{eV}, E_{H}=3.2 \mathrm{eV}\right)$ with an average efficiency of $13 \%$ would yield an $N_{0}$ of $\sim 60 \mathrm{~cm}^{-1}$. Assuming a $1 \mathrm{~cm}$ thick radiator of index 1.27 this would correspond to about 30 photoelectrons for a $\beta=1$ particle. Photoionization gas detectors operating in the UV achieve comparable or larger values.

In practice, the available radiator media are all dispersive. This chromatic distortion smears the emission angle of the photons as a function of energy and becomes a significant effect for imaging Cerenkov counters operating in the near vacuum ultraviolet region. For energies beyond the vacuum ultra violet, $n(E)$ becomes less than one and no Cerenkov photons are produced.

Cerenkov Counter Type

The information from Cerenkov radiation can be utilized in two distinct ways. This has given rise to two different types of counters. The first type, called a threshold counter, is designed to measure the light emitted by charged tracks with $\beta$ above threshold as given by Eq. (3.5). In its simplest form, it provides a binary flag as to whether the track velocity is above or below the threshold. In most modern counters, the number of Cerenkov photons is also measured approximately, allowing the separation range to be extended in momentum somewhat above the threshold for the heavier particle. The threshold counter is simple, and can be designed to cover large acceptance. To provide useful information for complex events, the detector must be split into many cells whose size is determined by the typical track separation. A counter with one radiator can usually only distinguish between two particle hypothesis over a rather limited momentum range. Additional counters containing radiators with different indexes of refraction can be used to extend coverage and distinguish between more than two particle types. 
The second type of device, called a focusing counter, not only counts the number of Cerenkov photons, but also measures their emission angle. Before the advent of position-sensitive photodetectors, such counters were built so that light was detected only when emitted within a particular narrow angular range. These devices, called differential counters, have very small acceptance in both angle and momentum space, and are of no interest in a B Factory secondary particle detector. They were used instead to identify particles in high momentum beamlines.

The development of th.. gasmins photocathnde cou pled to a position-sensitive gas detector ${ }^{14}$ has made the ring imaging focusing detector of the RICH (or CRID) type possible. In these detectors, both the number of photons and their emission angles are measured. These devices have very large acceptance in both angle and momentum, and excellent performance for closely spaced tracks. The effective resolution of an imaging counter in the region well above threshold is greatly superior to a threshold device as given by the derivative of Eq. (3.4),

$$
\delta_{\beta} \text { (Imaging) }=\frac{\sigma_{\beta}}{\beta}=\operatorname{tin}: A_{*} \cdot \frac{\sigma_{\theta}}{\sqrt{N_{p . e .}}},
$$

where $\delta_{\beta}$ is the percentage error on $\beta, \sigma_{\beta}$ is the error on $\beta$, and $\sigma_{\theta}$ is the measured angular error on each detected photon. This may be compared with the resolution of a threshold counter as calculated from Eq. (3.8)

$$
\delta_{\beta}(\text { T'hreshold })=\frac{\sigma_{\beta}}{\beta}=\frac{\tan ^{2} \theta_{c}}{2 \sqrt{N_{p . e .}}} .
$$

Thus, well above threshold, the resolution of the imaging counter is better than that of the threshold counter by a factor $R$;

$$
R=\frac{\tan \theta_{c}}{2 \sigma_{\theta}}
$$

The precise values of $R$ depend on a number of factors, including radiator, detector resolution and geome. try, particle momentum, etc. In principle, for an optimized gaseous detector it can exceed $250 .{ }^{13}$ More typi- 
Figure 16. The separation of a liquid CRID compared to that of a threshold counter filling the same space. The range of the threshold counter is chosen to give high momentum $\pi / K$ separation.

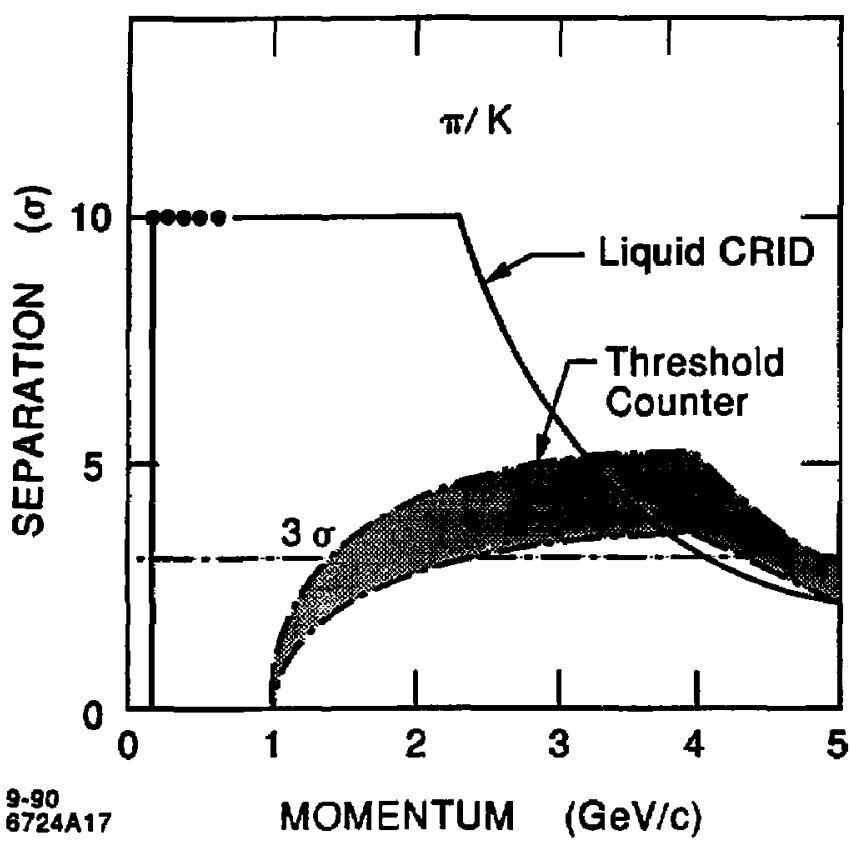

cally, in an existing detector, the SLD CRID, $R=140$ for the liquid radiator and 40 for the gas. ${ }^{15}$ Thus, the separation in an imaging counter of the SLD type between a light and heavy particle extends over a momentum range from just above the light particle threshold to over five times the threshold momentum of the heavy particle. In contrast, the momentum region of reasonable separation in a threshold counter extends from $\sim 20 \%$ above the light particle threshold to $\sim 20 \%$ above the heavy particle threshold. Figure 10 compares the separation expected for the liquid imaging counter (CRID) described in the following section with a threshold counter filling the same space. The index of refraction $n$ for the threshold counter is chosen to be $n=1.008$ so that the counter is optimized for $\pi / K$ separation in the high momentum regime $(1-4.5 \mathrm{GeV} / \mathrm{c})$ at the $\mathrm{B}$ Factory. $N$, is taken to be $60 \mathrm{~cm}^{-1}$ and $L$ is $15 \mathrm{~cm}$. The number of detected photons is then 14 , which is essentially the same as the standard CRID at $64^{\circ}$. The separation of a threshold de- 
vice is very asymmetric-it depends on whether the true particle is below or above threshold - so that translating separation into the equivalent Gaussian sigma $(\sigma)$ appropriate for the CRID is not well-defined. The shaded band in Figure 16 gives a spread which encompasses two different definitions; the highest is based on a likelihood test statistic and the lower on the number of standard derivations a true $\pi$ is separated from the $K$ hypothesis.

Clearly, either device can give adequate $\pi / K$ separation for the higher momentum region at a B Factory, provided that the segmentation of the threshold counter is sufficient. However, the CRID also has excellent performance in the region below $1.0 \mathrm{GeV}$; can identify protons over the whole region; and $e$ 's and $\mu$ 's at lower momentum as well.

\section{Photodetectors}

The development of the vacuum photomultiplier tube in the 1940's led to practical threshold Cerenkov coun. ters by providing efficient $(\sim 20 \%)$ detectors of single photons in the visible region. More recent tubes have bialkali cathodes, and special window materials, giving bandwidths into the vacuum ultraviolet and higher peak efficiency. Phototubes can be very fast devices, so that non-event associated background is not usually a problem. On the other hand, conventional tubes have no position resolution (except the tube aperture) and they are very sensitive to magnetic fields. More recently, tubes have been developed which perform rather well in fields up to 1 Tesla provided that the tube is oriented properly in the field.

Solid state photodetectors have also become available recently. Typically, their efficiency is quite high (>25\%) at longer wavelengths but cuts off in the UV below about $320 \mathrm{~nm}$. Perhaps the most promising device ior single photon detection is the avalanche diode, described below. At room temperature, their noise rate is high. Operation for single photon detection requires low temperatures and/or coincidence techniques. The sensitive area of these devices is also very small $\left(\sim 0.1 \mathrm{~mm}^{2}\right)$ and expensive per unit area covered. Somewhat larger 
devices may become available soon but flux concentration techniques are necessary if a large area counter is to be covered with such a photon detector.

The development of gaseous photocathodes contained within conventional time projection or multiwire chambers has enabled the inexpensive instrumentation of large areas with fine position resolution $(\sim 1 \mathrm{~mm})$, and made the rins imaging Cerenkov counter a reality. A number of high efficiency gases are known, but only those operating at the lowest photon energy [triethylamine (TEA), and tetrakis-dimethyl-amino-ethyline (TMAE)] are commonly used. Of these two, TMAE is the more widely used since it has significant efficiency $(\sim 40 \%)$ for photon energies below $\sim 7.5 \mathrm{eV}$ and carı therefore be used with quartz windows. TEA requires operation in the far vacuum ultraviolet which makes finding appropriate radiators difficult and demands that expensive materials, such as $\mathrm{CaF}$, and $\mathrm{MgF}_{2}$, be used for windows.

TMAE is a truly nasty gas. Nor only does it react very strongly with air, but it also attacks common construction materials; and the reaction products are quite danaging to the chamber. Thus, the detector materials must be carefully chosen and the host gas must be kept very pure. TMAE has a relatively long absorption length $(\sim 2.5 \mathrm{~cm})$ at room temperature, and the detector will therefore be rather thick and slow unless operated at high temperature. Conversely, room temperature TEA detectors can be quite thin since the absorption length is very short $(0.6 \mathrm{~mm})$.

Research aimed at finding suitable candidates for position-sensitive gas-filled detectors continues. ${ }^{16.17} \mathrm{En}$ couraging results have recently been obtained with a CsI cathode with an adsorbed TMAE layer. This cathode has efficiencies which exceed that of TMAE gas (> $40 \%$ below $7.0 \mathrm{eV}$ ) and appears to be stable and robust. This work is discussed in more detail below.

\section{Radiator Medium}

The radiator medium must conform to a number of criteria. It should have the correct refractive index for optimum particle identification separation; have low 
chromatic dispersion, to reduce the contribution of the chromatic error to the smearing of the emission angle and the threshold momentum; have high photon transmission over the detector bandwidth; have good radiation resistance so that the transmission does not deteriorate significantly on exposure to the few kilorad dose expected during the lifetime of the experiment; have negligible scintillation light compared to the Cerenkov light emission; have small radiation length, to reduce the probability of photon conversions in the detector; and should be mechanically and chemically robust.

The choices available in practice are heavily constrained. Room temperature gases have indices ranging from $1.000035(\mathrm{He})$ to $\sim 1.0018$ (e.g., $\mathrm{C}_{5} \mathrm{~F}_{10}$ at $30^{\circ}$ ). The gas pressure can be varied to give somewhat ligher indices but a pressure vessel is then required. The lowest index liquids (e.g., $\mathrm{C}_{6} \mathbf{F}_{14}$ ) candidates have an index of $\sim 1.27$, and transmit to photon energies of $\sim 7.0$ with modest chromatic dispersion. The indexes of refraction for solids in the visible span the range from $1.32(\mathrm{NaF})$ to about 6.0 . Of the solids, the alkali halides (e.g., $\mathrm{NaF}$, $\mathrm{LiF}, \mathrm{CaF}$ ) have the best combination of UV transmittance, low indexes, low dispersion, and ease of handling for use in imaging counters; but they are generally quite expensive. In any case, the best performing proximity counters in the B Factory environment use liquid radiators primarily because the index of refraction and chromatic dispersion are less. In the limit of no dispersion, it can be shown that the measurement error on the velocity $(\beta)$ scales with the index of refraction $(n)$ as

$$
\sigma_{\beta}=\frac{n \beta^{2} \sigma_{\theta}}{\sqrt{N_{0} L}} .
$$

Thus, for a fixed length radiator $(L)$ and constant quality factor $\left(N_{0}\right)$, the resolution scales wit $h_{1} n$, so that the resolution for a liquid counter with,$=1.28\left(\mathrm{C}_{6} \mathrm{~F}_{14}\right)$ exceeds that of a solid counter with $:=1.5(\mathrm{NaF}$ at $8 \mathrm{eV}$ ) by $\sim 15 \%$.

Indices of refraction between the high index gases at $\sim 1.0018$ and the low index liquids at $\sim 1.27$ are quite difficult to attain. Unfortunately, the indices needed for 
Table 2. Threshold momenta for $\pi$ 's, $K$ 's and $P$ 's for several indices of refraction. a B Factory threshold counter fall in this range as shown in Table 2.

\begin{tabular}{|l|c|c|c|}
\hline & \multicolumn{3}{|c|}{ Threshold Momentum } \\
\hline$n$ & $\pi$ & $K$ & $P$ \\
1.006 & 1.27 & 4.50 & 8.55 \\
1.008 & 1.10 & 3.89 & 7.40 \\
1.01 & 0.98 & 3.48 & 6.62 \\
1.02 & 0.69 & 2.46 & 4.7 \\
1.03 & 0.57 & 2.00 & 3.81 \\
\hline
\end{tabular}

One can reach indices in the lower end of the range ( $n \lessgtr 1.015$ ) using pressurized gas counters; the other approach is to use a "blown" $\mathrm{SiO}_{2}$ material called aerogel. New developments now allow aerogels to be manufactured with indices ranging between 1.0008 and 1.126 at $637.8 \mathrm{~nm}$. The transmittance is thought to be high but Rayleigh scattering leads to a very short bulk scattering length and consequent randomization of the Cerenkov angle. Thus, these radiators are not useful for ring imaging devices. However, if light collection can be made efficient, they might be very useful in a threshold counter optimized for the B Factory. The design of such a device is discussed in more detail below.

\section{The CRID-A Refenence Desig.}

$s$ discussed in the preceding section, ring imaging Cerenkov technology promises superior particle identification performance. ${ }^{14}$ Two different approaches to this technology are now under study-the long drift device (CRID or RICH) $)^{15,18}$ and the Fast RICH. ${ }^{19}$ Though they use similar principles to produce and focus the Cerenkov image, they differ in their approach to the problem of readout. Several long drift devices have now been constructed and the body of operation experience is becoming quite substantial. The Fast RICH technology is now in the prototype stage, and there is an active $R \& D$ program in place to investigate the problems associated with constructing a full scale system. 


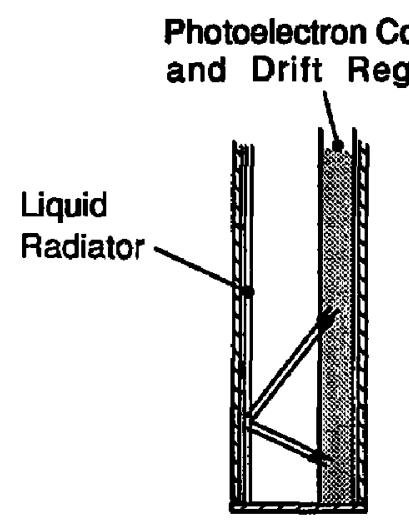

(a)

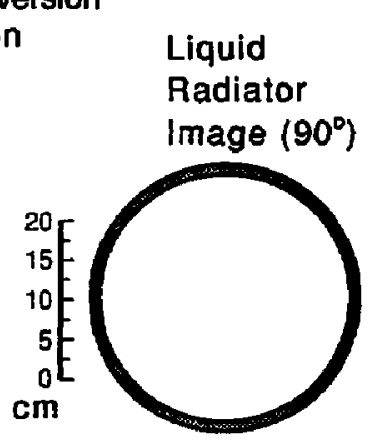

(b)

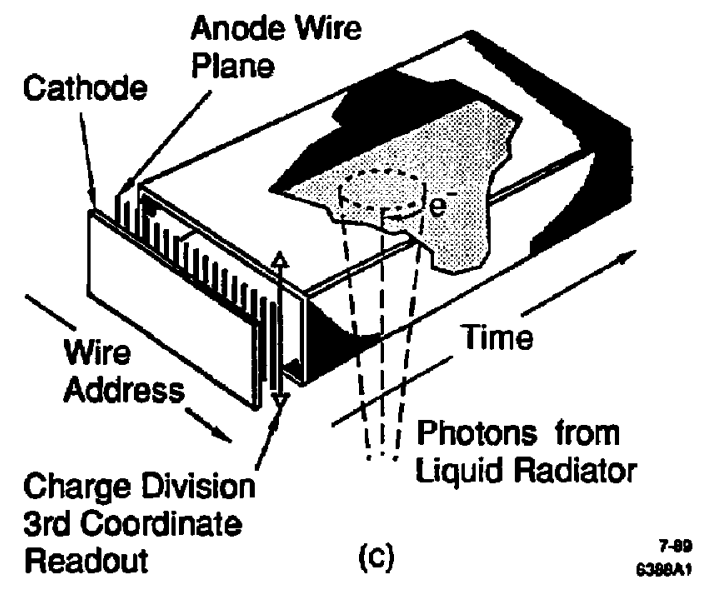

Figure 17.

(a) The CRID principle for a liquid radiator device; (b) size and thickness of a ring image produced at $90^{\circ}$ in a liquid radiator; (c) photon deteclor schematic.

In this section, we describe a candidate ring imaging device based on the long drift, Cerenkov Ring Imaging Detector (CRID) now being built for the SLD..$^{15}$ It is representative of the kind of particle separation performance that can be attained with a ring imaging counter, and thus can be considered as a reference study for imaging counters. Large inaging counters have operated in experiments for several years, and even though no large scale $4 \pi$ acceptance devices are now in full operation, 
two rather similar ring imaging Cerenkov detectors are now commissioning (the CRID for SLD and the RICH for DELPHI ${ }^{18}$ ); their performance in prototypes is wellestablished ${ }^{20,21}$; and within about a year, their operation in the colliding beam environment should be understoad; so that it will soon be clear if the promise will be met in practice.

The concern is that the long drift technology may not translate easily into the high rate environment of the $B$ Factory. This issue will be partially addressed in the following sections, but not all facets are fully solved, and further $R \& D$ is required in some cases to prove that suggested solutions are really viable, or perhaps to develop a more robust approach. A CRID is a rather complex device; it contains a significant amount of material which unavoidably affects the performance of the electromagnetic calorimetry. A few suggestions for improvement are offered, but large improvements will require major changes to the technology, such as the Fast RICH.

A schematic design for a CRID, a discussion of its performance and mass, and a brief discussion of costs are given below. For this exercise, we will make use of existing techniques developed for the SLD to the maximum extent possible. Changes in some of these features that might be desirable in a real device will be discussed as appropriate.

\section{Principle of CRID Operation}

As shown in Figure 17, when a charged particle passes perpendicularly though a thin liquid radiator, it produces a cone of light whose angle depends on the particle velocity and the index of refraction of the liquid. If this Cerenkov light is allowed to propagate some distance before hitting the photon detector, it will form an image which is said to be "proximity focused" on the detector. For our geometry, using liquid $\mathrm{C}_{6} \mathrm{~F}_{14}$ with an index of refraction $n=1.277$, the proximity focused circle for a relativistic $(\beta=1)$ particle is around $17 \mathrm{~cm}$ radius. There are typically around 25 photoelectrons produced for tracks which cross the liquid radiator near 
(a)

$B \bar{B}$ Detector

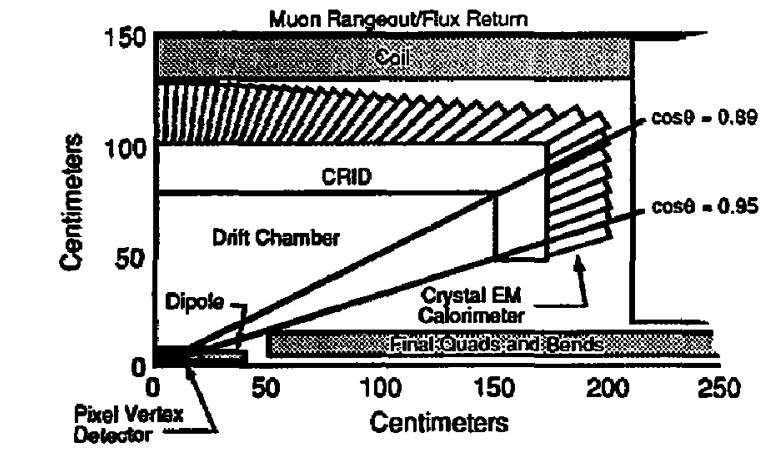

(b)

Calorimeter
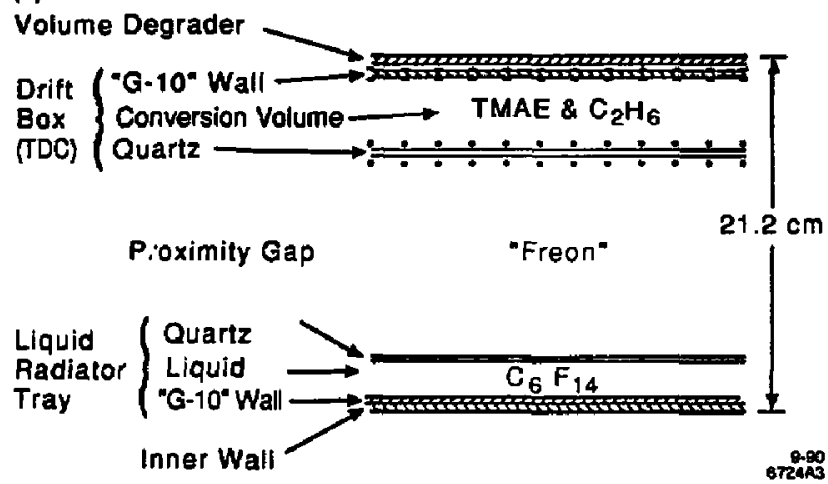

Figure 18. Conceptual geometry for a CRID:

(a) Jocation in the detector; (b) schematic cross section.

the normal, but some photons are lost by total internal reflection for angles exceeding $\sim 13^{\circ}$ so that, more typically, there are around 14 photoelectrons observed. The Cerenkov photons from the charged particle of interest pass through quartz windows in the liquid radiator cell and the detector box, and are converted by photoionization of gaseous TMAE (Tetrakis Dimethyl Amino Ethylene), which has a very high quantum efficiency in the range 170 to $220 \mathrm{~nm}$. The photoelectrons drift at constant velocity in a uniform electric field along the box until they arrive at a proportional wire detector. The position of the photoelectron conversion must be measured in three-space. Two coordinates come from the drift time of the electrons and the wire address of 


\begin{tabular}{|l|c|}
\hline \multicolumn{2}{|c|}{ SUMMARY OF CRID PARAMETERS } \\
\hline 1. Solid Angle Coverage (re-entrant design) & $92 \%$ \\
2. Angular Coverage (Barrel) & $37^{\circ}-90^{\circ}$ \\
3. Angular Coverage (End Cap) & $18^{\circ}-37^{\circ}$ \\
4. Radiator Material & $\mathrm{C}_{6} \mathrm{~F}_{14}(1 \mathrm{~cm})$ \\
5. Index of Refraction (at $6.5 \mathrm{eV}$ ) & 1.277 \\
6. Window Material & Quartz \\
7. Photocathode Material & TMAE Gas at $20^{\circ} \mathrm{C}$ \\
8. Focusing Method & Proximity \\
9. Proximity Gap & $13 \mathrm{~cm}$ \\
10. Cerenkov Angle (for $\beta=1)$ & $672 \mathrm{mr}$ \\
11. Radius of Cerenkov ring (for $\beta=1)$ & $17 \mathrm{~cm}$ \\
12. Cerenkov Quality Factor $N_{0}$ & 65 \\
13. Number of Photoelectrons (for $\beta=1)$ & $25\left(\mathrm{at} 90^{\circ}\right)$ \\
14. Momentum Threshold (for five Photoelectrons at $90^{\circ}$ ) & \\
$e$ (cut oft by $p_{t}$ ) & $\sim 1 \mathrm{MeV} / \mathrm{c}$ \\
$\mu$ & $0.17 \mathrm{GeV} / \mathrm{c}$ \\
$\pi$ & $0.23 \mathrm{GeV} / \mathrm{c}$ \\
$K$ & $0.8 \mathrm{GeV} / \mathrm{c}$ \\
$p$ & $1.5 \mathrm{GeV} / \mathrm{c}$ \\
15. Particle Separation Range at $64^{\circ}(3 \sigma$ Level) & \\
$e / \pi$ & 0.2 to $1.200 \mathrm{GeV} / \mathrm{c}$ \\
$\mu / \pi$ & 0.2 to $0.8 \mathrm{GeV} / \mathrm{c}$ \\
$\pi / K$ & 0.23 to $4.1 \mathrm{GeV} / \mathrm{c}$ \\
$K / p$ & 0.8 to $6.9 \mathrm{GeV} / \mathrm{c}$ \\
\hline
\end{tabular}

Table 3. Summary of CRID parameters. the hit wire, while charge division on the proportional wires determines the conversion depth in the box.

\section{Geometry}

Conceptually, a CRID would sit just outside the central tracker, in front of a TOF (if any) and the calorimeter as indicated in Figure 18(a). The barrel CRID can start at any radius (or at any $z$ distance for an end cap device) determined only by the outer dimensions of the tracker. However, the thickness of the device is essentially "fixed" at around $20 \mathrm{~cm}$. That is, the performance of a thinner device will be compromised. An end cap can 
be re-entrant to make an approximately hermetic device in polar angle. However, cracks between the liquid radiator boxes will lead to approximately 1-3\% acceptance loss in azimuth.

A cross section of the CRID is shown in Figure 18(b). Starting from the inside, a particle first passes through an inner wall which in this design is a 7 mm HEXCELL cylinder with aluminum skins. It then passes through the $4 \mathrm{~mm}$ thick G-10 back of the liquid radiator tray, through $1 \mathrm{~cm}$ of $\mathrm{C}_{6} \mathrm{~F}_{14}$, and out through the $4 \mathrm{~mm}$ thick quartz window of the liquid radiator tray into a $13 \mathrm{~cm}$ drift region filled with a UV transmitting gas with good HV properties (such as a light fluoro-carbon). It then passes through a $3 \mathrm{~mm}$ quartz window into the $4.4 \mathrm{~cm}$ photon conversion region of the drift box (containing $\mathrm{C}_{2} \mathrm{H}_{6}$ plus TMAE), through the $3 \mathrm{~mm}$ back wall of the drift box, and finally through about $\boldsymbol{z} \mathrm{mm}$ of epoxy equivalent in a HV degrader of the ALEPH/DELPHI type. ${ }^{18,22}$

Table 3 summarizes the properties and parameters of the proposed B Factory Cerenkov Ring Imaging Device.

\section{Performance}

The particle separation capabilities of the CRID depend primarily on the precision with which the Cerenkov angle is measured, which, in turn, depends on the angular precision of each photoelectron measurement and the number of photoelectrons detected. A detailed discussion of the various sources of measurement error and photoelectron production rates is given elsewhere. ${ }^{15,23}$

In general, the number of photons is largest for particles that hit the radiator at normal incidence. For angles of incidence greater than $\approx 13^{\circ}$, some photons get cut off by total internal reflection in the radiator. This photon loss is partially compensated by the increasing path length the particle travels in the radiator, so that the typical number is $\approx 60 \%$ of the maximum. The number of photoelectrons $N_{\text {p.e. }}$ observed in the detector for a particle traveling at normal incidence is

$$
N_{\text {p.e. }}=N_{0} L \sin ^{2} \theta_{c}
$$


where $N_{0}$ is the Cerenkov quality factor (about $65 \mathrm{~cm}^{-1}$ ) for a $\mathrm{C}_{6} \mathrm{~F}_{14}$ radiator and a TMAE photocathode, $\theta_{\mathrm{c}}$ is the Cerenkov angle, and $L$ in the length the particle travels in the radiator. For the configuration under discussion, $N_{p . e .}=25$ at normal incidence.

The important contributions to the total error are; (a) geometrical error, which is dominated by the relative depth of the radiator and the proximity drift space; (b) detector measurement error, which includes diffusion, measurement granularity, and nonuniformities in electron drift in the detector; (c) multiple scattering error in projecting the track measured in the central tracking chamber out to the CRID; (d) momentum smearing error due to the bending of the particle in the radiator; and (e) chromatic aberration error in the radiating liquid.

The contributions from various sources as a function of momentum at a track angle of $64^{\circ}$ are shown in Table 4.

\begin{tabular}{|c|c|c|c|c|c|c|c|c|}
\hline & & \multicolumn{6}{|c|}{ Angular Resolution per Photoelectron (mrad) } & \\
\hline$P$ & $\begin{array}{l}\theta_{C}(\pi) \\
(\mathrm{mrad})\end{array}$ & $N_{\text {p.e. }}$ & Geometry & $\begin{array}{c}\text { Detector } \\
\text { Measurement }\end{array}$ & $\begin{array}{l}\text { Multiple } \\
\text { Scattering }\end{array}$ & $\begin{array}{c}\text { Momentum } \\
\text { Smearing }\end{array}$ & $\begin{array}{l}\text { Chromatic } \\
\text { Aberration }\end{array}$ & Total \\
\hline 0.5 & 622.2 & 12.5 & 7.3 & 5.7 & 2.9 & 0.7 & 5.3 & 3.2 \\
\hline 1.0 & 660.0 & 13.8 & 7.3 & 5.7 & 1.4 & 0.3 & 5.0 & 2.9 \\
\hline 1.5 & 666.4 & 14.0 & 7.3 & 5.7 & 0.9 & 0.2 & 4.9 & 2.9 \\
\hline 2.0 & 668.8 & 14.1 & 7.3 & 5.7 & $0 . \bar{t}$ & 0.2 & 4.9 & 2.8 \\
\hline 3.0 & 670.5 & 14.2 & 7.3 & 5.7 & 0.5 & 0.1 & 4.9 & 2.8 \\
\hline 4.0 & 671.2 & 14.2 & 7.3 & 5.7 & 0.4 & 0.1 & 4.9 & 2.8 \\
\hline 5.0 & 671.4 & 14.2 & 7.3 & 5.7 & 0.3 & 0.1 & 4.9 & 2.8 \\
\hline
\end{tabular}

Table 4. Angular resolution (mrad) per photoelectron as a function of momentum at $64^{\circ}$.
The sizes of each particular contribution depend on track angle and momentum, but ty'pically the largest single contribution $\left(\sim 7.3 \mathrm{mr}\right.$ per photoelectron at $64^{\circ}$ ) comes from the geometrical term. Typically, the combined contribution from all other terms is about the same size, so that the error per photon is $\sim 11 \mathrm{mr}$. The size of the geometrical term can only be reduced either by making the radiator thinner, which loses photoelectrons. 


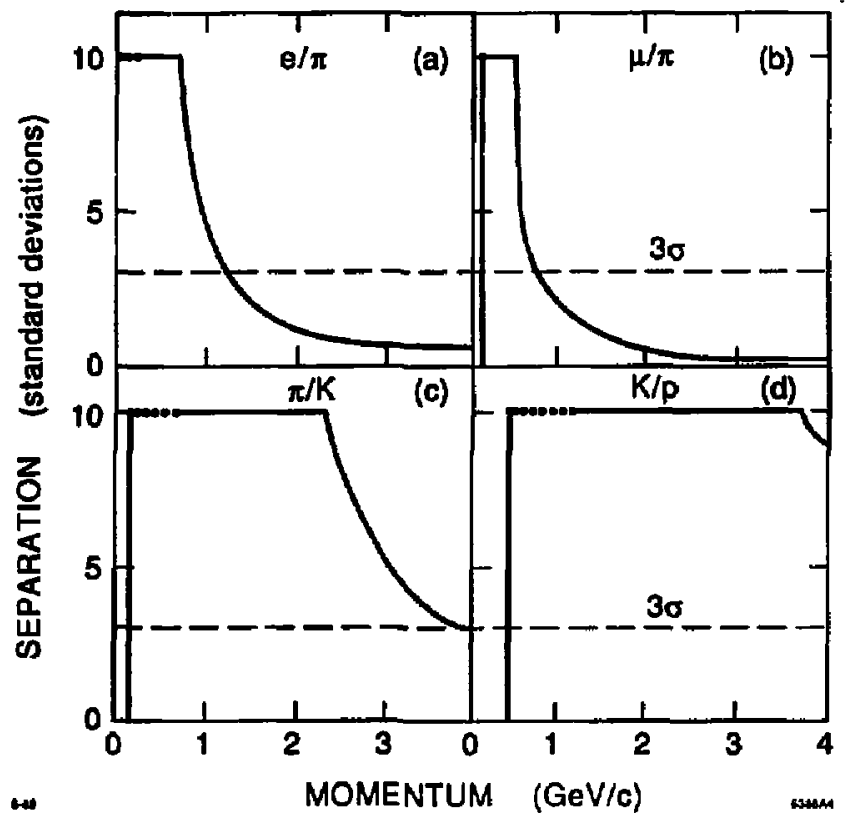

Eigure 19. Particle separation capability of the liquid CRID system at $64^{\circ}$. The curves are saturated at $10 \sigma$ (see text). In the dotted regions of the curves, only the lighter particle emits light in the liquid. There are thresholds shown at low momentum below which the lighter particle emits too few photons in either quartz or liquid to be reliably separated (see text). The separation is shown for: (a) $e / \pi$; (b) $\mu / \pi$; (c) $\pi / K$; and (d) $K / p$.

or by making the drift space longer, which makes the overall counter thicker.

The particle separation capabilities of the CRID are shown in Figure 19. The Monte Carlo particles for results shown here entered the radiator at a polar angle of $64^{\circ}$ relative to the beam line, so that these plots include the typical loss of photons due to total internal reflection that occurs when the particles enter the radiator at oblique angles. The vertical scales give the separation between different kinds of particles in "standard deviations $(\sigma) . "$ It is "saturated" at $10 \sigma$, since the "Gaussian" separation probabilities estimated here will surely be unreliable for very large estimated separations. The hadronic separations are generally excellent, for $\pi / K$, for example, exceeding $10 \sigma$ from near threshold up to $\approx 2.3 \mathrm{GeV} / \mathrm{c}$, and falling to $3 \sigma$ around $4 \mathrm{GeV} / \mathrm{c}$. The CRID also provides excellent low momentum lepton identification which complements the calorimetry and muon tracking chambers very well. At the $3 \sigma$ level, $e / \pi$ 
separation is achieved up to $1.2 \mathrm{GeV} / \mathrm{c}$, and $\mu / \pi$ separation up to $0.8 \mathrm{GeV} / \mathrm{c}$.

There are regions at the lowest momenta where both particles are below threshold. This is a significant effect only for the $K / p$ case. $K$ threshold in the liquid is at $620 \mathrm{MeV} / \mathrm{c}$. The $K / p$ separation extends down to $\approx 480 \mathrm{MeV} / \mathrm{c}$ using radiation from the quartz. For lower momentum, the number of photons produced by the kaon becomes too small for reliable separation, and, in any case, below $430 \mathrm{MeV} / \mathrm{c}$ neither particle ernits any photons. However, in cases where particles are too slow to be separated by the Cerenkov it is very simple to obtain $d E / d x$ separation since one or both particles are far into the $1 / \beta^{2}$ region where even very modest amplitude information on energy loss will provide excellent separation. There is also a low momentum cutoff due to particle curvature in the magnetic field, which depends on the CRID radius, the magnetic field, and the polar angle of the particle. For example, a device at $80 \mathrm{~cm}$ radius in a 10 KG field is unable to identify a particle traveling normal to the magnetic field below $\sim 170 \mathrm{MeV} / \mathrm{c}$. Again, this "hole" in the CRID coverage can be filled with very modest $d E / d x$ information from the tracking chamber in the $1 / \beta^{2}$ region.

\section{SLD CRID Technology}

As was shown above, the performance of a CRID using SLD technology with a liquid radiator matches the partjcle ID requirements for $\pi / K / p$ extremely well and also gives significant help to the calorimeters for low momentum $e$ and $\mu$ identification. The device, as described above, is essentially a liquid only version of the SLD CRID except

1. there is an outer volume degrader, which is necessary to keep the space requirements down if a long drift configuration is chosen for the detector;

2. this CRID can be run at room temperature, which makes the gas and fluid systems much simpler with little effect on performance for a liquid radiator only system; 


\begin{tabular}{|l|l|}
\hline \multicolumn{2}{|c|}{ RADIATION LENGTH OF THE } \\
CRID COMPONENTS \\
\hline INNER WALL & $0.018\left(L / L_{R A D}\right)$ \\
LIQUID RADIATOR TRAY & $0.105\left(L / L_{R A D}\right)$ \\
PROXIMITY GAP & $0.000\left(L / L_{R A D}\right)$ \\
DRIFT BOX & $0.044\left(L / L_{R A D}\right)$ \\
OUTER WALL & $0.036\left(L / L_{R A D}\right)$ \\
\hline TOTAL & $0.203\left(L / L_{R A D}\right)$ \\
\hline
\end{tabular}

Tarle 5. Radiation length of the SLD CRID components.

\begin{tabular}{|l|l|r|r|}
\hline \multicolumn{3}{|c|}{ CRID CONSTRUCTION COSTS } \\
\hline BARREL & VESSEL & 448 & \\
& LIQUID RADIATORS & 212 & \\
& DRIFT BOXES & 566 & \\
& $e^{-}$DETECTORS & 191 & \\
& SPARES & 97 & \\
& E.D.\&.I. & 500 & \\
BARREL TOTAL & & & 2014 \\
\hline END CAP & & & \\
& VESSEL & 323 & \\
& LIQUID RADIATORS & 52 & \\
& DRIFT BOXES & 138 & \\
& e- DETECTORS & 153 & \\
& SPARES & 34 & \\
END CAP TOTAL & E.D.\&.I. & 360 & \\
\hline FLUID SYSTEMS & & & 1060 \\
\hline ELECTRONICS & & & 560 \\
\hline CRID TOTAL & & & 1440 \\
\hline
\end{tabular}

Table 6. Estimated cost of a $B$ Factory CRID.

3. a fast readout system is necessary to handle the $1 \mathrm{KHz}$ trigger rates (see below).

The CRID contains approximately $20 \%$ of a radiation length of material distributed as shown in Table 5 .

Significant reductions in this mass inventory are difficult so that substantial effects on precision electromag- 
netic calorimetry seem unavoidable in any detector which contains a CRID of the SLD type. Moreover, the device has a "bad geometry" in that much (about 50\%) of the mass is located a long way from the calorimeter. A few possibilities for modifications to the technology which could both reduce the total mass in the device and improve its distribution are discussed below. However, large improvements (i.e., 50\% or more) seem unlikely in this technology.

A CRID is a rather expensive device to construct. In general, the component costs scale approximately with the area covered, whereas the electronic costs scale linearly with the radius. For a given detector size, costs can be scaled from actual SLD costs. If we assume a B Factory CRID just outside a central tracker of $80 \mathrm{~cm}$ radius by $250 \mathrm{~cm}$ length, with an end cap CRID starting at a $30 \mathrm{~cm}$ radius, the estimated costs are as given in Table 6.

\section{Electronics}

The SLD CRID electronics are matched to the properties of the SLC environment: for example, the trigger rate is slow (typically $1 \mathrm{~Hz}$ ); there are $\approx 5 \mathrm{~ms}$ between machine pulses; and the particles are highly collimated into jets, so that high hit densities and pulse pair resolution are important issues. At the $B$ Factory, on the other hand, the trigger rate is fast (perhaps $1 \mathrm{KHz}$ ); there are $\approx 5$ ns between machine pulses; the typical particle densities are relatively low; and there are only large radius liquid rings so that the number of real hits per box will usually be small. The total number of hits should be dominated by background.

These differences in machine environment lead to several modifications in the electronics. Conceptually, the front-end (preamp) electronics can be just like the SLD CRID (with a different shaping time) but a different readout is required. Full wave form digitization (as in the SLD) is possible, but would be expensive at these data rates, and it is probably unnecessary. Simple considerations of hit densities expected at the B Factory suggest that four hits per wire should be sufficient (see below). 

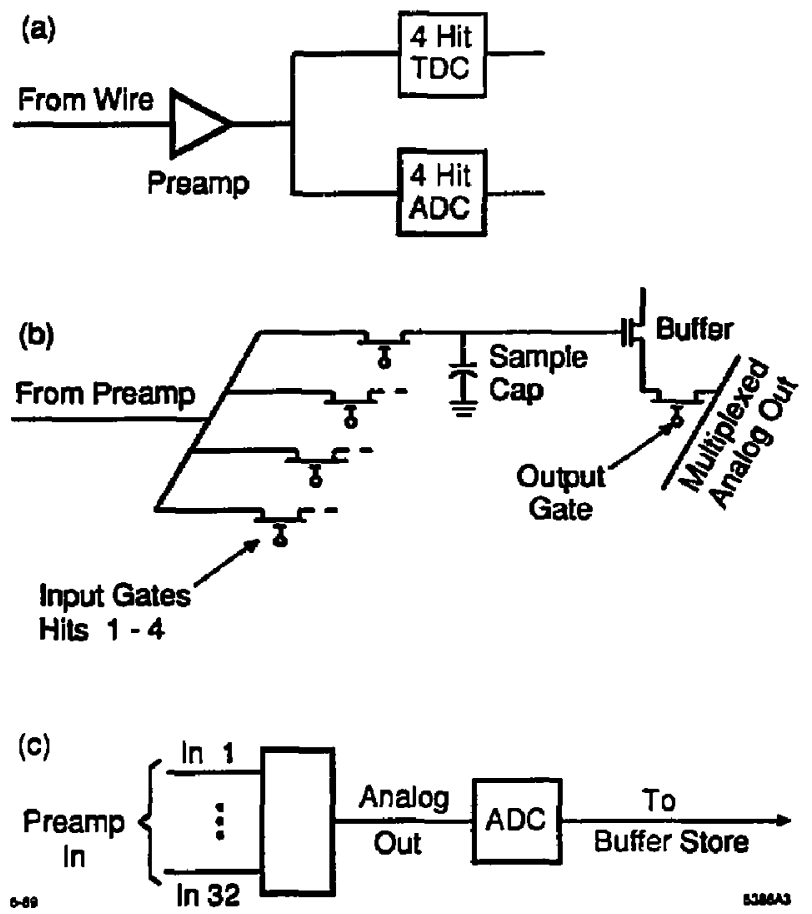

Figure 20. Conceptual desiga for CRID readout: (a) four-hit electronics for each wire end; (b) parallel-in, serial-out analog memory unit ( 1 of 32 channels); (c) preamp multiplexing scheme.

At these hit densities, it is probably much cheaper to readout each wire end by a combination TDC/ADC.

A four hit version is indicated schematically in Figure 20(a). The TDC portion is quite straightforward. There are a variety of ways one might implement the ADC. For example, it could use a simple modification of the SLD CDU ${ }^{15}$ module as shown in Figures 20(b) and $20(\mathrm{c})$. This is a simple analog storage device with parallel-in, serial-out architecture which has 4 cells per channel. The exact level of multiplexing into the ADC depends on the readout time desired and on the ADC digitizing speed. In particular, with the 32 preamp multiplexing shown in Figure 20(c), an ADC with $1.5 \mu \mathrm{s}$ digitizing speed leads to $20 \%$ deadtime at a projected $1 \mathrm{KHz}$ trigger rate. 


\section{Problems of the CRID Technology}

There are two major difficulties associated with the CRID technology which we discuss more fully below. First, since the CRID is massive and sits between the interaction region and the calorimeter, it will affect the low energy performance of the calorimeter. These effects are discussed more fully in chapter on calorimetry. We will briefly describe below a few ways to reduce this problem.

Secondly, photoelectrons in the CRID drift a long distance (taking $\approx 25 \mu \mathrm{s}$ ). Backgrounds from beam individual crossings add during the total drift time, leading to uncorrelated backgrounds. This, in turn, leads to questions about the sensitivity of the chambers to average current, and to possible difficulties in identifying tracks.

None of these problems seem to be fatal to the CRID . technology described, but they do have uncomfortable implications for triggering and operations.

CRID Mass and Its Distribution.

A CRID of the SLD type affects the low energy performance of any calorimeter which follows it because (1) the radiation length of the device is rather large (about $20 \%$ ), and (2) the mass is poorly distributed in that much of it is located a substantial distance in front of the calorimeter. Thus, not only does a photon have a substantial interaction probability $(\sim 17 \%)$, but the conversion products can also get lost, either by scattering or by bending in the magnetic field. The scattering loss goes like $L$ (the distance from the conversion point to the calorimeter) while the loss due to bending ${ }^{24}$ in the the magnetic field goes like $L^{2}$. For a conventional CRID in a $1 \mathrm{~T}$ field, the loss from the magnetic field becomes significant at around $200 \mathrm{MeV}$, while that coming from the scattering begins around $25 \mathrm{MeV}$. Thus, the low energy performance of the calorimeter can be improved by making the CRID thinner, and by either reducing the total amount of material or improving the overall material distribution by placing the maximum amount of material close to the calorimeter. It is helpful, in addition, to 
either make the counter a good charged particle tracker or to place a layer of tracking between the CRID and the calorimeter, so that the energy of converting photons can be corrected, or, at worst, events with converting photons can be cut from the data sample.

A number of suggestions for possible improvements were made during the $B$ Factory workshop which deserve further study. ${ }^{24-27}$ A sample of these are briefly described below. However, to dute, all modifications which have been suggested trade-off improved low energy photon properties in the calorimeter for degraded particle identification performance.

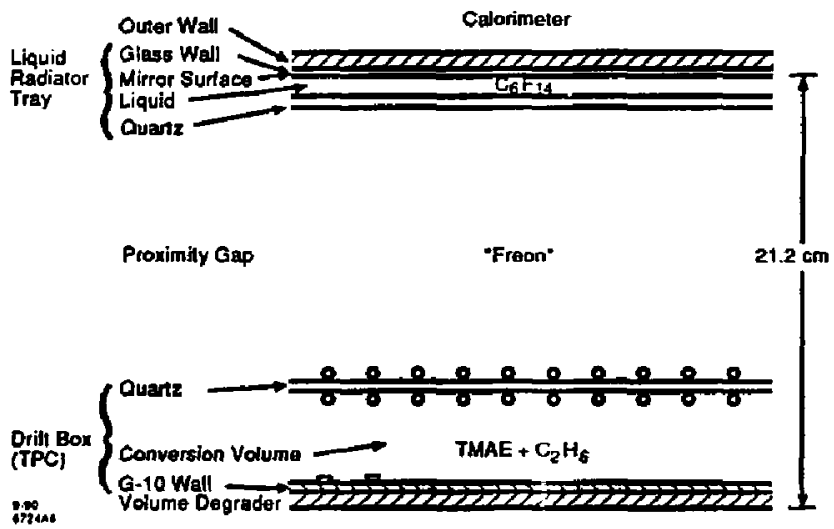

Figure 21. Cross section for a CRID with a refected proximity gap.

At first sight, the scheme shown in Figure 21 appears useful in improving the mass distribution. A mirrored surface in the liquid radiator tray reverses the direction of the Cerenkov photons so that the most massive element (the liquid tray) has been placed near the calorimeter. Unfortunately, in actuality this hes almost no effect on the mass distribution, as can be seen from the table above. The radiator plus wall are about $12 \% L_{R A D}$ total whereas the TPC plus degrader are $9 \%, L_{R A D}$; there is little net gain from turning the systerz around. Morcover, jt degrades the performance substantially compareri to the normal configuration, as can be :een in Figure 22, because the number of Cerenkov phot, , ns is reduced by 
Figure 22. Particle separation $(\pi / K)$ capabil. ity of several different liquid CRID systems at $64^{\circ}$; solid line for the standard configuration (Figure 18); dashed line for the reflected proximity gap counter (Figure 21) and the "honeymoon" configuration ( $F$ igure 23); dotdash line for the active proximity gap configuration (Figure 24).

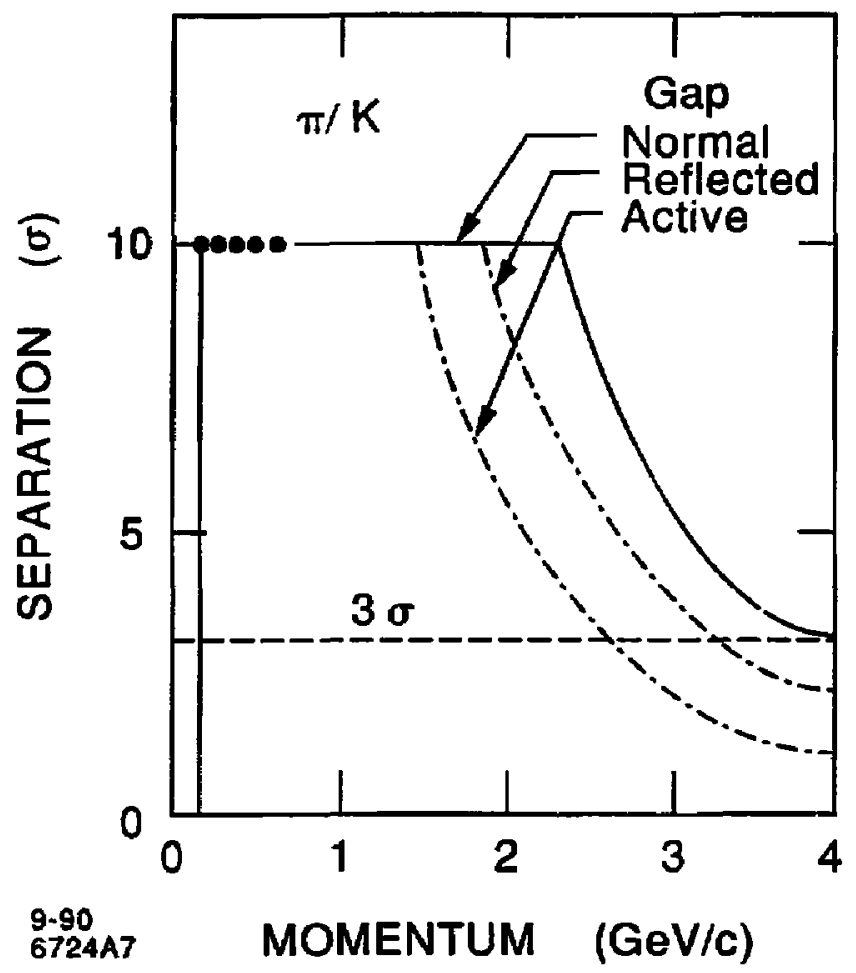

about $40 \%$ by absorption and reflection. The performance of the counter in the presence of background will degrade for the same reason.

A more amusing "honeymoon" configuration which marries the detector and radiator is shown in Figure 23. Its performance is essentially the same as the that of the device shown ir. Figure 21. The mass of one nontransmitting window can be saved, but this savings is compensated by the material needed for an independent reflecting surface. The main advantage is that the proximity gap gets "used" twice so that the overall counter becomes about $14 \mathrm{~cm}$ thick rather than about $21 \mathrm{~cm}$. On the other hand, the distribution of mass is very bad.

As a final example, Figure 24 shows a configuration for an active proximity gap counter. The TMAE vapor 


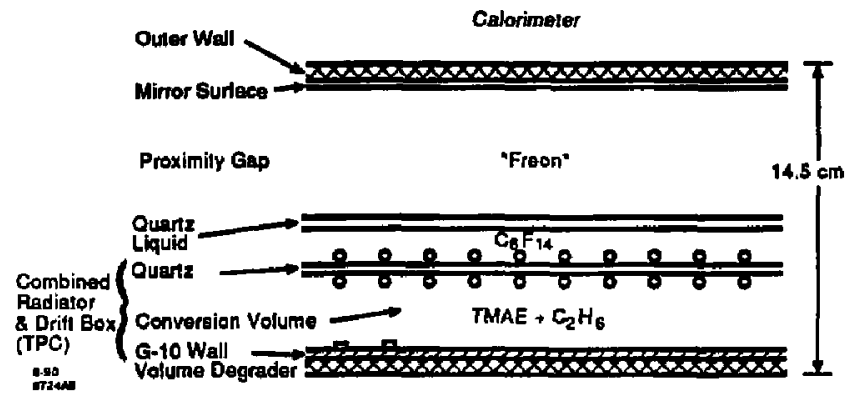

Figure 23.

Schematic cross section for a "honeymoon" CRID with a combined radiator tray and detector and $\mathbf{a}$ reffected proximity gap.

pressure has been reduced to give an absorption length in the conversion gap of $20 \mathrm{~cm}$, which gives near the optimum performance for this total counter thickness. One quartz window is eliminated, but the materjal savings are compensated for by the need for an additional volume degrader. The performance given in Figure 22 is substantially worse than that of the normal configuration. The main advantage is that this counter is active over most of its volume so that even low energy conversion products can be reliably recognized. However, the sensitivity of the single electron detectors to background might keep this feature from full exploitation, if restrictive triggers are used for the CRID (see the following section).

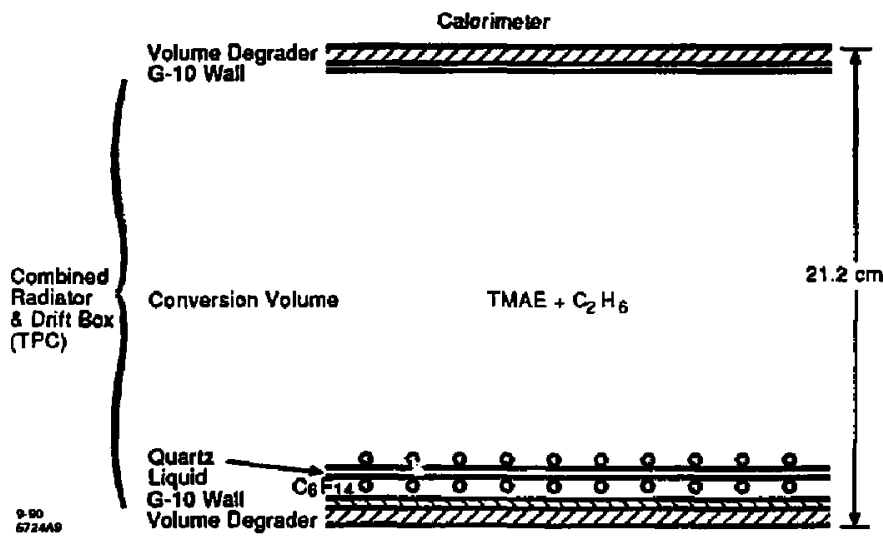

Figure 24.

Schematic cross section for an active proximity gap CRID with a cormbined radiator tray and detector and a long absorption length conversion volume. 
Detector Performance and Background Sensitivity

The high rate environment at a $\mathrm{B}$ Factory can affect the long drift TPC photon detector of the CRID in a number of ways. The primary concerns are that positive ions build-up in the drift volume will distort the field and the measured positions for the conversion Cerenkov photons; and that the single eleciron detectors, which in the CRID are PWCs with $7 \mu \mathrm{m}$ carbon filament sense wires operating with ethane plus TMAE gas, will not have a lifetime commensurate with the expected time of experimental operation.

The distorting effects of positive ion build up in TPCs are well known, and generally are dealt with by a gate at the detector-drift region interface which collects the positive ions before they enter the drift region. ${ }^{28}$ When gated closed, electrons from the drift volume are also prevented from entering the avalanche region of the chamber. Since the positive ions drift much more slowly than the electrons, the gate can be made to collect $100 \%$ of the positive jons (in principle) that are produced by avalanches of electrons which come from the TPC drift volume provided that the trigger rate is properly controlled. However, at the very high rates of a $B$ Factory, the ions coming from electrons produced by background in the PWC itself could become troublesome, since they leak back into the TPC drift volume when the gate switches open. In this case, the avalanche gain also needs to be gated, as is discussed below.

The addition of TMAE gas to the usual PWC mixtures has been shown to reduce the lifetime of chambers by factors of at least 100 and more typically around $1000 .{ }^{29}$ Thus, the lifetime expected for CRID PWCs in a B Factory is of great concern and was discussed extensively during the workshop. ${ }^{27,30,31}$ A number of approaches were attempted. For example, attempts were made to scale the TPC experience at PEP to the B Factory, ${ }^{27,30}$ while other studies looked at backgrounds more directly.

More research in this area is needed. The tentative conclusion is that the CRID sensitivity can be made similar to or better than that of the CDC provided that 
the fast ( $\sim 2 \mu \mathrm{s}$ or better) trigger rate can be kept under $1000 \mathrm{HZ}$. With this gating ratio, the net charge collected on the sense wire begins to be dominated by avalanches coming from electrons which are initially produced on the PWC side of the gate. Thus, it is important to develop a means of lowering the gain during the time the TPC gate is off. Since the average multiplicity in events at the B Factory are rather small, another factor of 5 can be gained by gating only those TPCs in the vicinity of charged tracks. Finally, since the gain of the CRID TPCs can be quickly recovered by the wire heating technique ${ }^{29}$ which burns off polymerized deposits on the sense wires, it would appear, in a sense, that the CRID is substantially more robust than the CDC. Of course, this does not guarantee the performance of either device.

In summary, the single issue of greatest concern in translating the SLD technology to the B Factory detector remains the single electron detection chamber lifetime. Studies to date indicate that it can be controlled if (1) care is given to PWC chamber gating; (2) the CRID gate-on trigger rate can be kept under $1000 \mathrm{~Hz}$; and (3) the CRID detection PWC's are routinely rejuvenated by wire heating. However, a more robust solution would be to develop a single electron detector for the TPC which is less sensitive to ageing effects. Some potential candidates are now under development. ${ }^{32,33}$

\section{Hypothesis Testing and Background Sensitivity}

Both SLD and DELPHI studies have shown that the hypothesis testing method of Cerenkov analysis is quite insensitive to rather large numbers of background hits. The readout is essentially fully segmented into $\approx 10^{6}$ 2-D pixels so that the number of hits that can be well separated per event is very large. True signal hits are on average separated by many pixels, so that they are not confused even in the presence of large numbers of background hits. Moreover, analysis is attempted only after points are correlated with tracks found in the central chamber. No independent "pattern recognition" is attempted. Instead, points are associated with tracks; tested against all five possible hypotheses; and the best 
Figure 25. Loglikelihood differences $\left(L_{\pi}-L_{K}\right)$ for $10002.5 \mathrm{GeV} / \mathrm{c}$ particles; the end bins include the overflow: (a) true kaons, $B=0.0$; (b) true pions, $B=0.0$; (c) true kaons, $B=1.0$; (d) true pions, $B=1.0$; (e) true kaons, $B=10.0$; (f) true pions, $B=10.0 ;(\mathrm{g})$ true kaons, $B=40.0$; (h) true pions, $B=40.0$.

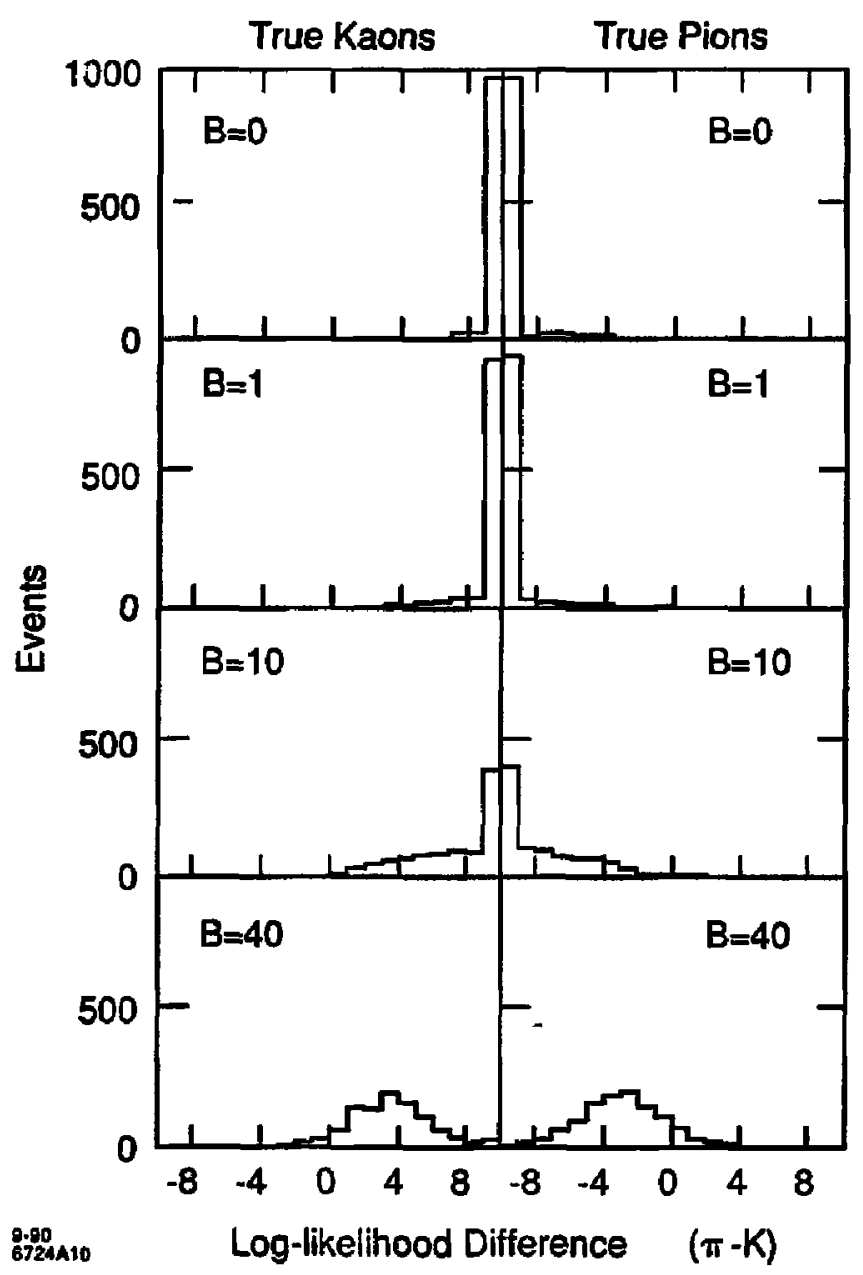

one chosen. Provided that the geometry and performance parameters of the device are well understood, and that the background can be simply modeled, the results are indeed very robust as is demonstrated briefly below. These results are discussed in more detail elsewhere. ${ }^{33,34}$

This study uses a Monte Carlo, which throws Cerenkov photons for the true particle and adds random back. ground hits. A likelihood ratio test of the SLD/DELPHI 


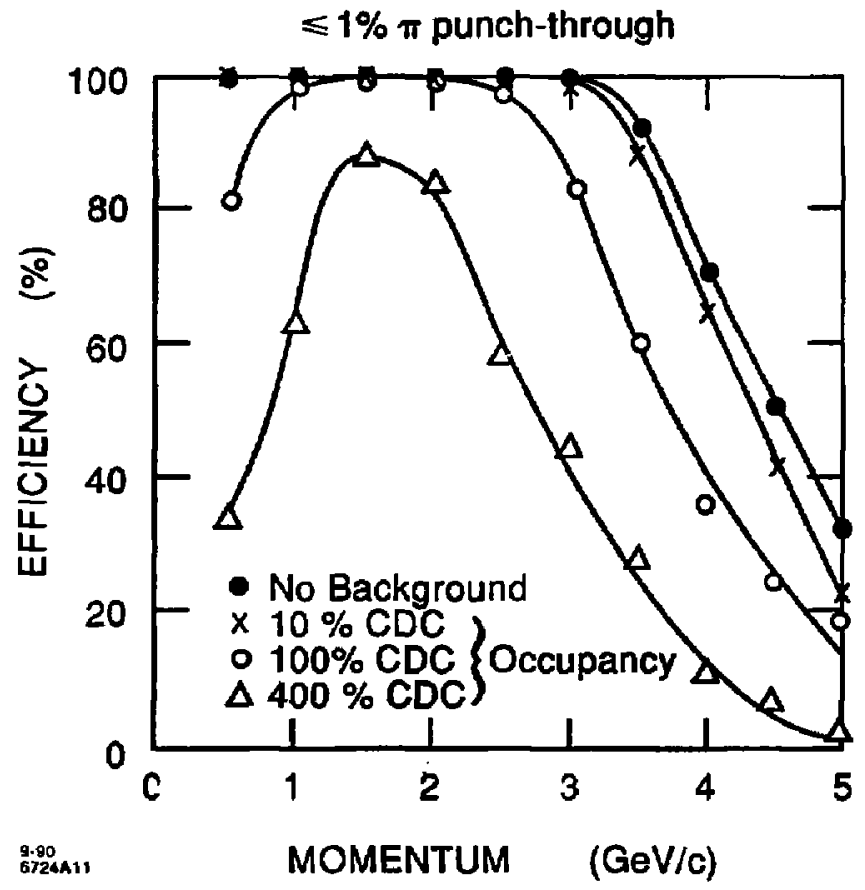

Figure 26. Efficiency for labeling a true $K$ as $\mathbf{A} K$ as a function of momencum when the maximum $\pi$ punchthrough is limjted to $1 \%$.

type is then employed by performing, for each track, a test for each possible hypothesis. The likelihood ratios obtained can then be compared for the possible hypotheses. For simplicity, the study performed here only considers the $\pi$ and $K$ hypothesis, and log-likelihoods are used instead of likelihoods. The function calculated for each hypothesis has the form:

$$
\begin{aligned}
L_{H Y P}= & -N_{H Y P} \\
& +\sum_{i=1}^{N} \ln \left[N_{H Y P} \times e^{-\left(\theta_{1}-\theta_{H Y P}\right)^{2} / 2 \sigma_{i}^{2}}+B\left(\theta_{i}\right)\right],
\end{aligned}
$$

where $N_{H Y P}$ is the number of photons expected for the h ypothesis; $\theta_{i}$ is the measured Cerenkov angle for the $i^{\text {th }}$ photon; $\theta_{H Y P}$ is the expected Cerenkov angle for the hypothesis; $\sigma_{i}$ is the error on the C'erenkov angle measured for the $i^{\text {th }}$ photon; and $B\left(\theta_{i}\right)$ is a background function. 
Figure 27. Efficiency for labeling a true $K$ as a $K$ as a function of background and $\max -$ imum $\pi$ punchthrough probabilities.

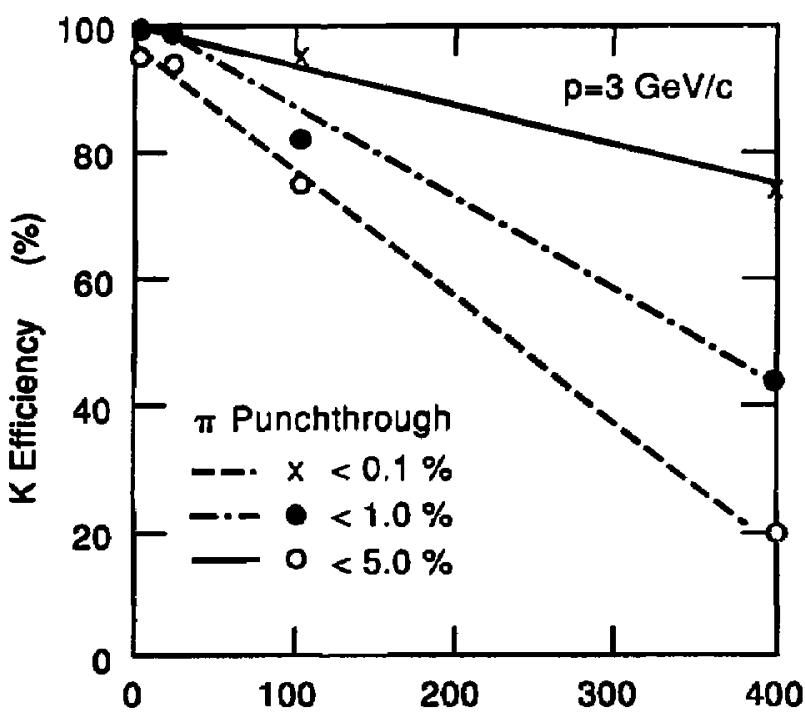

Background (Equivalent CDC Occupancy) $\underset{\text { 8724a12 }}{(\%)}$

For simplicity, the background as taken to be flat and was normalized so that a value of 1 gives $1.0 \mathrm{hit}$ in a $\pm 3 \sigma$ road about the true hypothesis. By way of comparison, if the background is dominated by interacting soft photons, then 1 hit is approximately equivalent to $10 \%$ occupancy in the $\mathrm{CDC} .{ }^{33,34}$

The differences between the log-likelihoods for $2.5 \mathrm{GeV} / \mathrm{c}$ particles are shown in Figure 25 for a variety of different background conditions. At low values of the background, there is no overlap between the true $\pi$ and true $K$ distributions, whereas as the background gets very large the overlap becomes much more significant. A clean sample can then only be obtained by reducing the efficiency for the wanted particle. However, the highest background level given $(B=40)$ is very large indeed and much more than 10 times the tolerable background in the CDC. The efficiency for $K$ identification (labeling a true $K$ as a $K$ ) is shown in Figure 26 as a function of momentum and background when the maximum $\pi$ punch-through probability is limited to $1 \%$, whereas 
Figure 27 shows the relationship between efficiency and background at a single momentum $(3.0 \mathrm{GeV} / \mathrm{c})$. The background is given in equivalent CDC occupancy using the scaling noted above. The identification is affected very little by background well beyond the maximum tolerable levels acceptable by the CDC.

\subsection{CRID Summary}

The CRID technology described above meets the performance requirements for particle ID at a B Factory very well. In addition to providing superior $\pi / K / p$ separation it also fills in the lepton ID holes which are left by the calorimetry and muon tracking techniques. However, it adds $\sim 20 \%$ of a radiation length of material in front of the calorimeter and takes about $20 \mathrm{~cm}$ of space. The device using SLD technology is rather insensitive to backgrounds at the pattern recognition level. The integrated charge sensitivity appears to be tolerable but only with $\epsilon$ rather restrictive fast trigger, and excellent gating efficiencies. New single electron detectors are under study which could make the hardware less sensitive to backgrounds.

\section{A Design for Particle ID Using a Fast RiCH}

O ne very attractive potential solution to the problems posed by the use of a TPC in an imaging Cerenkov is a new type of imaging Cerenkov which is called the Fast RICH. It was proposed some time ago in connection with the design of detectors for the symmetric B Factory at the PSI and for LEAR. ${ }^{19,35}$

A generic schematic of this device is shown in Figure 28. The essential difference between this device and the CRID is the replacement of the three-dimensional readout of the CRID TPC by a two-dimensional readout via a pad system. The photoelectron drift is kept very short (in the radial direction in a solenoidal geometry). The necessity for reading out three dimensions is 


\section{Calorirneter}

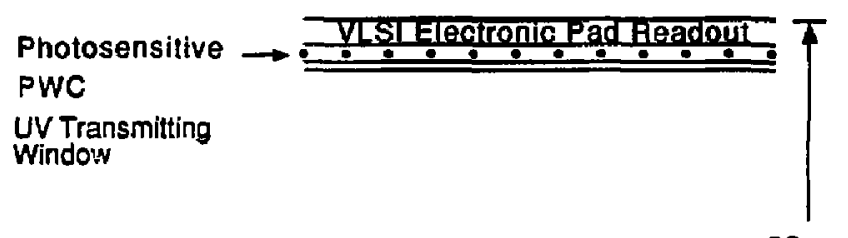

Proximity Gap

Figure 28.

Schematic cross section for a Fast RICH.

eliminated by using a photocathode with a short absorption length. Since the photoelectron which is produced by the Cerenkoy photon has a short drift length, it also $l$ as a short drift time; the device is fast; and it has been froposed for use at high luminosity hadron colliders as viell as $B$ Factories. ${ }^{36}$

A schematic of the device proposed for the BMF detector at PSI is shown in Figure 29. It includes a number of novel features in addition to the fast photoelectron readout which were necessary implementation details at the time it was proposed. In pa:ticular, the photocathcde chosen is TEA, which has the short $(0.5 \mathrm{~mm})$ absorption length required at room temperature. The radiator proposed is $\mathrm{NaF}$, since it transmits into the far I $V$ range required by the TEA photo-efficiency spectrum. Because $\mathrm{NaF}$ has a hirh index of refraction in the LV region wherr TEA is photosensitive, Cerenkov photons emitted by fast particles uraveling at nearly normal i incidence are trapped in the radiator by total internal reflection. Therefore, a section of radiator in the $90^{\circ}$ region is tilted ${ }_{*}^{\prime} 10^{\circ}$. The device contains approximately $: 0 \% L_{R A D}$ of material for particles at normal incidence.

A number of issues of design and construction must I e solved before such a device can be built. A major prototyping effort is now well underway. In particular, the VLSI electronics chips for reading out the pads have now 
been made and successfully tested; tests of TEA quantum efficiency have been made; and a full scale test of a B Factory prototype in a beam to confirm operations, electronics performance, resolution, and speed will be performed in the fall of $1990 .^{16}$

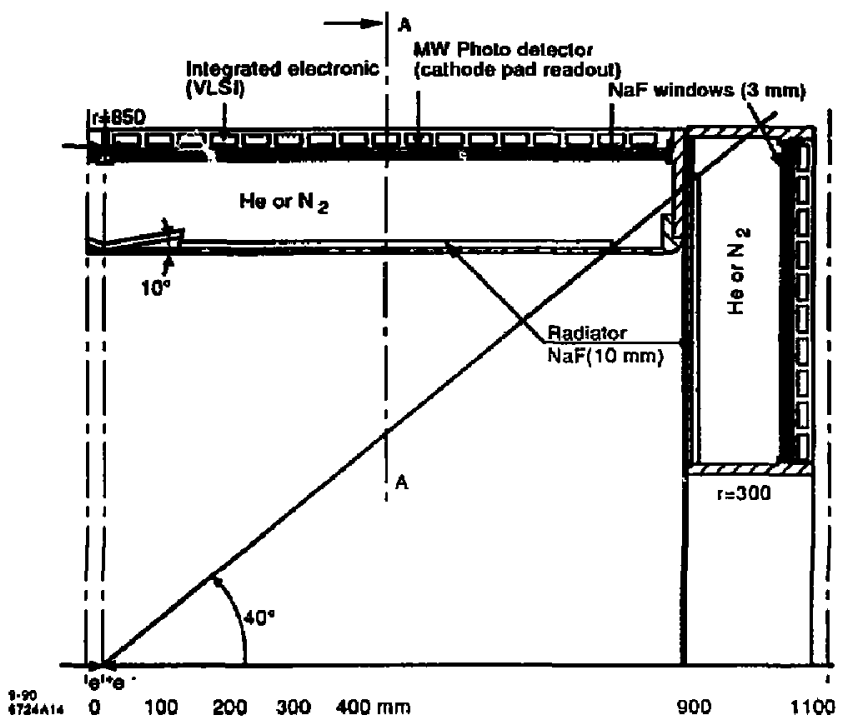

Figure 29.

Schematic cross section for the $B M F$ (PSI) Fast RICH.

This device originally was thought to provide good $\pi / K$ separation up to $3 \mathrm{GeV} /$ c. $^{16,19.35}$ Recent measurements of the refractive index of $\mathrm{NaF}$ crystals demonstrate that $\mathrm{NaF}$ is more dispersive than was predicted from the 1-pole fit extrapolation used previously, and the predicted $3 \sigma$ cutoff of $\pi / K$ separation is now limited to $2.2 \mathrm{GeV} / \mathrm{c}^{16}$ This is ccnfirmed by a simulation of the performance of a $\mathrm{NaF}$ device, using a different detector model, by Coyle. ${ }^{33,37}$ Though such performance is marginally adequate for a symmetric rollider, such as PSI, it is clearly insufficient for the asymmetric machine described in this report.

Recently, a study of reflective UV photocathodes with gas phase electron extraction has been underway as part of the LAA project at CERN..$^{16.17}$ 
Figure 30. The measured quantum efficiency of a $500 \mathrm{~nm}$ thick CsI photocathode as a function of photon wavelength $\lambda_{i}$ the solid line is the TMAE gas-phase efficiency; the dashed line is the CoI efficiency; and the open squares give the effcjepcy for a CsI photocathode $\left(30^{\circ} \mathrm{C}\right)$ on which an $a b-$ sorbed film of TMAE has been deposited.

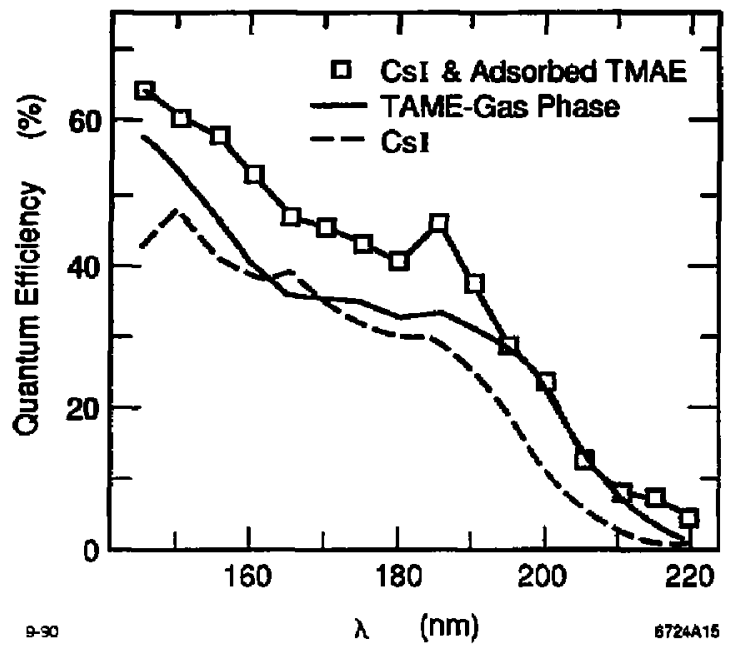

A CsI photocathode with an adsorbed TMAE film has been observed to have an efficiency equal to or exceeding the TMAE gas-phase region of interest as shown in Pigure 30. It should be well adapted to Fast RICH counters because of its isochronous signals and room temperature operation. Further studies of photocathode lifetime, feedback, and operation in the presence of positive ion build up are necessary, but results to date are very impressive.

The existence of this new photocathode leads to a proposal for a new design for a Fast RICH using a liquid $\mathrm{C}_{5} \mathrm{~F}_{12}$ radiator and a reflective $\mathrm{CsI}+\mathrm{TMAE}$ photocathode as shown schematically in Figure $31 .^{16}$ The CsI + TMAE cathode operates in the 6-7 eV photon region where fluoro-carbon liquids and quartz windows transmit well, so that expensive, dispersive solids like $\mathrm{NaF}$ are not needed for radiators or windows. The VLSI readout chips developed for the BMF NaF+TEA Fast RICH discussed above can be used for pad readout. Hexane gas is added to the methane chamber atmosphere to eliminate the UV feedback photons above $7.0 \mathrm{eV}$.

The $\mathrm{C}_{5} \mathrm{~F}_{12}$ liquid is slightly superior to the $\mathrm{C}_{6} \mathrm{~F}_{14}$ used in the CRID, and the $\pi / K$ identification range for this device extends well above $4.0 \mathrm{GeV} / \mathrm{c}$ (to $5.5 \mathrm{GeV} / \mathrm{c})^{16}$ 


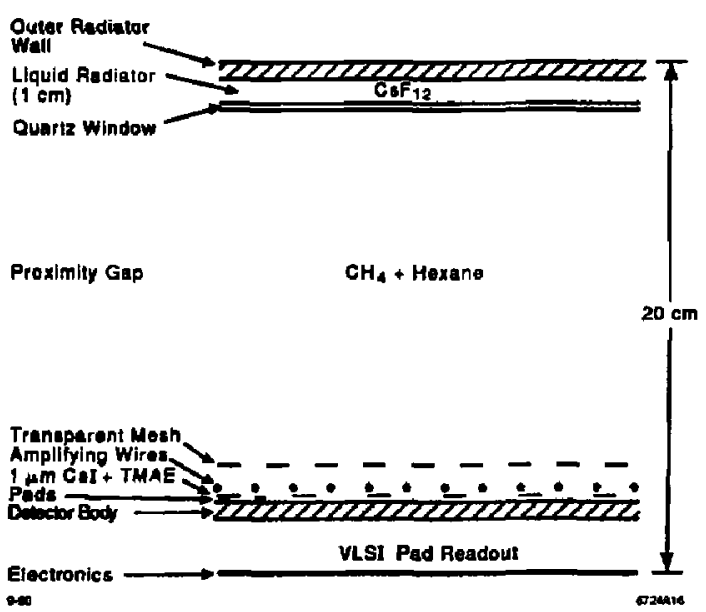

as required for an asymmetric B Factory. However, the device must be run at room temperature (or slightly below) to stay below the $28^{\circ} \mathrm{C}$ boiling temperature of the liquid. $\quad \mathrm{C}_{6} \mathrm{~F}_{14}$ liquid radiator of the type discussed in the preceding section would also give adequate performance. The amount of material in this device also appears to be less than required for the CRID, and a reflected proximity gap appears to improve the material distribution. The estimate for particles at normal incidence is about $12 \% L_{R A D}$ in total, and the contribution from the material farthest from the calorimeter, i.e., the detector and readout chips, is about $2.5 \% L_{R A D}$.

In summary, the Fast liquid RICH is a very promising idea which has the performance specifications required a the asymmetric B Factory without the background and lifetime issues that rajse concerns for the CRID discussed in the preceding section. Additional $\mathrm{R} \& \mathrm{D}$ is now underway, ${ }^{16}$ which should soon indicate if the promise of this approach can be met in practice.
Figure 31. Schematic cross section for a jiquid radiator Fast RICH with a refected proximity gap and a CsI plus TMAE cathode. 
A particle identification sys: tem at an asymmetric $B$ Factory must provide $\pi / K$ separation to high momentum.
Candidate Cerenkov systems are based on threshold and ring-imaging lechniques.

\section{Afrogel Threshold Čerenkov Counters}

\subsection{Introduction}

A lthough excellent $K / \pi$ separation at high momentum is the most important requirement of a particle identification system at a $B$ Factory, such a system has to satisfy additional criteria. First, it has to operate in a magnetic field, which limits the choices of read-out systems. Second, in order to design a highly efficient and uniform detector, it is necessary to cover almost the entire solid angle hermetically. Third, the entire device should be built with low-density materials amounting to a few percent of a radiation length (r.l.), in order to maintain the good energy resolution of the electromagnetic calorimeter for all photons. Finally, the costs to built such a detector have to be acceptable.

The Cerenkov effect-based particle id systems under discussion can be divided into threshold devices and ring imaging devices. The Ćerenkov ring imaging counters certainly provide the best particle identification in the required momentum range. Besides high costs, their biggest disadvantage is, however, the large amount of inactive nuaterial in front of the electromagnetic calorimeter. It amounts to $\sim 20 \%$ of a r.l., thus affecting the energy resolution of $\sim 15 \%$ of all photons. This has motivated us to search for altelnatives. Interesting candidates are Cerenkov threshold counters using silica aerogel as racliator, because these low density devices provide good $K / \pi$ separation up to high momenta. They were first proposed by E. Lorenz for the PSI and CERN B Factory projects. ${ }^{38}$

We will discuss a particle identification system using silica aerogel threshold Cerenkov counters. After reviewing the main properties of silica aerogel, we will propose differcnt cell designs, discuss their light yields and efficiencies, and present the results from a Monte Carlo simulation. We also present preliminary test results and address technical issues leading to improvements. We then discuss the particle identification performance of aerogel threshold C'erenkov counters and compare it to 


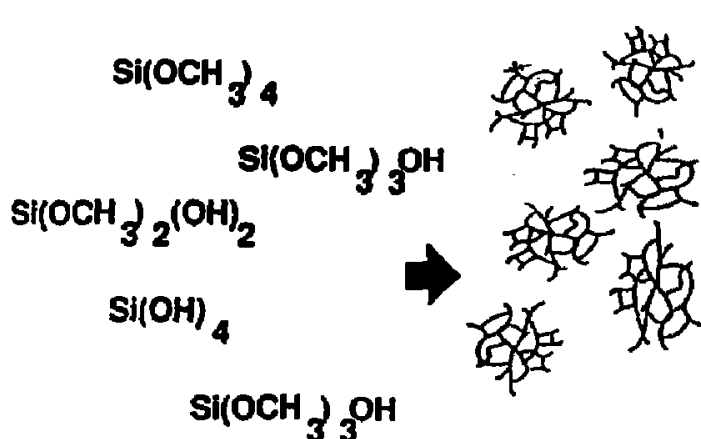

Reactants
Clusters

\section{3-20 nm}

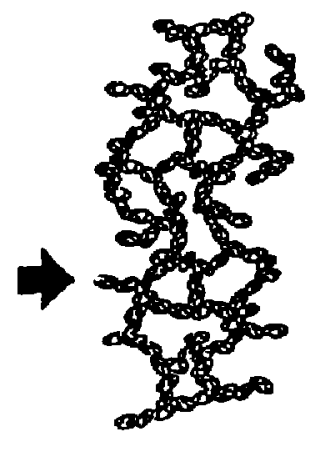

Gel

\section{THE ULTRASTRUCTURE OF THE CROSSLINKED GEL IS CONTROLLED THROUGH SOLUTION CHEMISTRY}

that of CRID/RICH counters. Finally, we give conclusions and an outlook on future R\&D.

\subsection{Properties of Silica Aerogel}

Silica aerogel is a glass-like structure which consists of amorphous $\mathrm{SiO}_{2}$. In the classical synthesis it is produced in a single-step process by hydrolysis and condensation of silicon alkoxides ${ }^{39,40}$ :

$$
\begin{gathered}
\mathrm{Si}(\mathrm{OR})_{4}+4 \mathrm{H}_{2} \mathrm{O} \stackrel{\text { bastecatalyst }}{\Longleftrightarrow} \mathrm{Si}(\mathrm{OH})_{4}+4 \mathrm{ROH} \\
\mathrm{Si}(\mathrm{OH})_{4} \stackrel{\text { basecatalyst }}{\Longleftrightarrow} \mathrm{SiO}_{2}+2 \mathrm{H}_{2} \mathrm{O}
\end{gathered}
$$

where $R$ represents an acylgroup $\left(\mathrm{CH}_{3}, \mathrm{C}_{2} \mathrm{H}_{5}, \ldots\right), \mathrm{Am}$ monia is typically used as a catalyst. During condensation, $3-20 \mathrm{~nm}$ size clusters of $\mathrm{SiO}_{2}$ are formed, which
Figure 32. Formation of silica aerogel.

Silica aeragel is produced by hydrolysis and condensation of silicon alkoxides. 


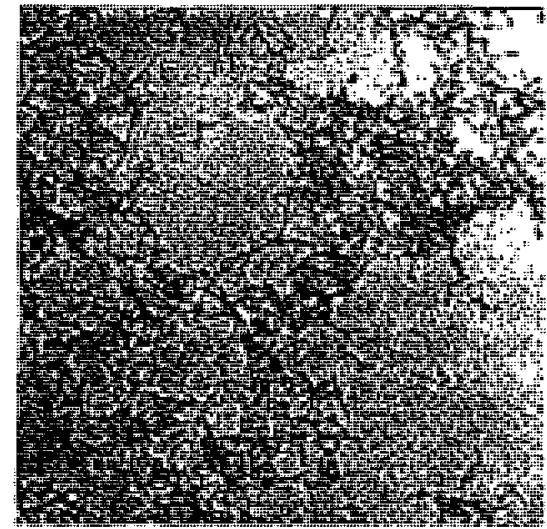

TEM of ULD aerogel $\left(0.008 \mathrm{gm} / \mathrm{cm}^{3}\right)$

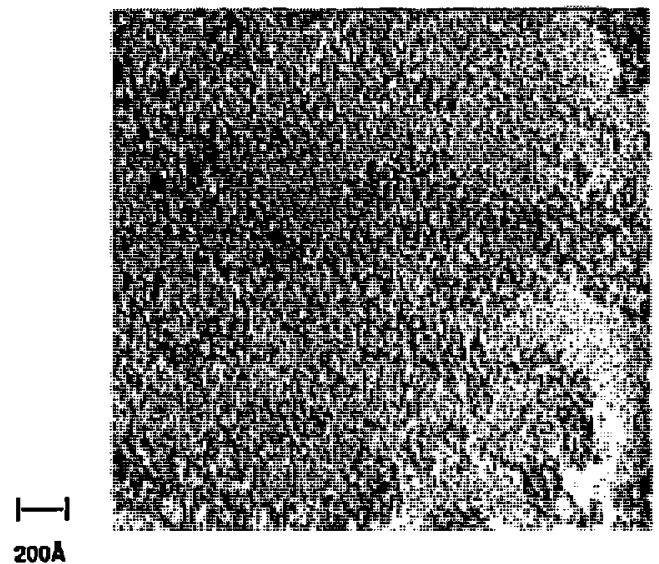

TEM of base $/ T M O S\left(0.04 \mathrm{gm} / \mathrm{cm}^{3}\right)$
Figure 33. Transmission Electron Microscope (TEM) pictures of aerogels produced in a two-step process and in a single-step process.

High-temperature and lowtemperature techniques exist for supercritical extraction. then partially collapse forming a structure of colloidal connected spheres, as shown in Figure 32. The sphere sizes are typically 4-6 $\mathrm{nm}$ in diameter, whereas the dianeters of the pores range from 30 to $50 \mathrm{~nm}$. Since the density range aclieved in this production process is rather limited $\left(22 \mathrm{mg} / \mathrm{cm}^{3}-280 \mathrm{mg} / \mathrm{cm}^{3}\right)$, a new two-step process has been developed at Lawrence Livermore $\mathrm{Na}$ tional Laboratory (LLL) to produce both lower-density and higici-density aerogel. ${ }^{41}$ In the first step a partially hydrolyzed, partially condensed silica is formed. This is achieved by supplying only 2 moles $\mathrm{H}_{2} \mathrm{O}$ per mole of $\mathrm{Si}(\mathrm{OR})_{4}$ lor the liydrolysis, controlling the reaction by an acid catalyst and replacing all produced alcohol with a non-alcoholic solvent. In the second step both the hydrolysis and condensation are completed by adding water and o base catalyst. The density is controllable by the amount of non-alcoholic solvent. Due to the acid catalyst, silica acrogel produced by the two-step process has a different morphology, as shown in Figure $33 . .^{42}$ It consists of chains of clusters, where the average chain diamelcr is 2 nm.

Aerogel is dried by supercritical extraction to eliminate the cflects of surface tension, ${ }^{42}$ which tend to collapse the solid. Two different techuieques are in use for 
the production of large aerogel monoliths. In the hightemperature technique, the solvent is evaporated at $260^{\circ} \mathrm{C}$ in an autoclave under a pressures of $\sim 140$ atm. This process, which takes up to 12 hours, produces liydrophobic aerogel. An additional heat treatinent at $450^{\circ} \mathrm{C}$, however, is frequently applied to remove alcohol groups still bound to the silicon. In this process, aerogel becomes hydrophylic. In the low-temperature technique the solvent is exchanged with liquid $\mathrm{CO}_{2}$. This procedure can take up to a week depending on the size of the monolith. The extraction is then done at a temperature of $35^{\circ} \mathrm{C}$ and a pressure of $\sim 80$ atm. The extraction, which takes 12-24 hours, produces hydrophyllic aerogel. This technique is well suited for the production of aerogels mixed with wavelength shilters.

Silica aerogel is the lowest-density. transpartut. manmade bulk material. Aerogels wilh densities $p$ ranging from $3 \mathrm{mg} / \mathrm{cm}^{3}$ to $600 \mathrm{mg} / \mathrm{cm}^{3}$, can be produced. The relationship between the density and the index of refrac. tion $n$ is given by:

$$
\rho=\frac{n-1}{0.21}\left(\frac{\mathrm{g}}{\mathrm{cm}^{3}}\right)
$$

These densities correspond to refractive inclices of $n=$ 1.0006 and $n=1.126$ at $637.7 \mathrm{~mm}$, respectively, which yield thresholds of $0.27 \mathrm{GeV} / \mathrm{c}$ to $3.4 \mathrm{~T}$ Gel/c for pions and $0.95 \mathrm{GeV} / \mathrm{c}$ to $12.34 \mathrm{GeV} / \mathrm{c}$ for kaons. Since the production of large homogeneous blocks is straightforward, it is interesting to explore the use of these aerogels for radiators in threshold Cerenkov counters.

Density fluctuations are controllable ${ }^{42}$ to $\Delta \rho / \rho \leq$ 1\%; most inhornogeneities occur within a few millimeters of the edges. The uncertainty of the momentum threshold due to density fluctuations is approximately given by:

$$
\frac{\Delta p}{p}=\simeq \frac{1}{2} \frac{\Delta \rho}{\rho}
$$

Since both the pore and sphere dimensions are close to wavelengths in the UV, aerogel scatter's light like a
Silica aerogel with refractive indices between $n=1.0006$ and $n=1.126$ can be produced. 
Cesenkov light is Rayleighscattered in aerogel.

Figure 34. Transmission spectra from a $4 \mathrm{~mm}$ thick sample oi aerogel catalyzed from letramethylorthosilicate (TMOS) supercritically dried at $270^{\circ} \mathrm{C}$ and 110 bars and from a $12 \mathrm{~mm}$ thick sample of aerogel catalyzed from tetraethylorthosilicate (TEOS), supercritically dried but not heat treated.
Rayleigh scatterer. For a homogeneous and isotropic aerogel, the Rayleigh cross section is given by:

$$
\frac{d \sigma}{d \Omega}=a^{6} k^{4} \frac{\left|n^{2}-1\right|^{2}}{\left|n^{2}+2\right|^{2}} \frac{1}{2}\left(1+\cos ^{2} \theta\right)
$$

where $\theta$ is the scattering angle, $a$ is the size of the scatterer and $k=2 \pi / \lambda$ is the wave number. Thus, for lowdensity aerogels $(n-1 \ll 1)$, the scaltering cross section is basically proportional to $(n-1)^{2}, \lambda^{-4}$ and $1+\cos ^{2} \theta$. Since the Cerenkov spectrum falls as $\lambda^{-2}$, the original directionality of the Cerenkov photons is lost, after passing a few centimeters of aerogel. However, in the visible and infrared light region aerogel is highly transmissive as shown in Figure $31 .^{40}$

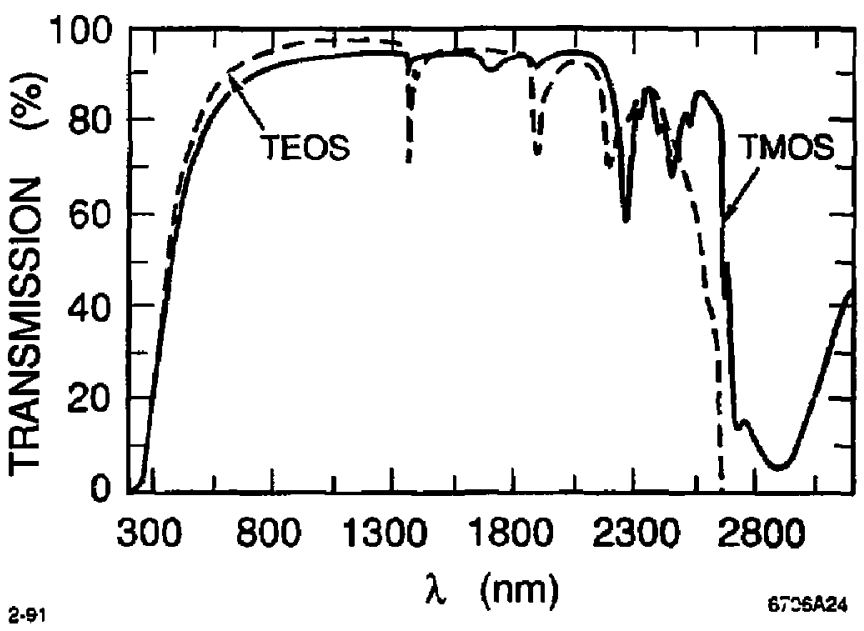

Aerogcl is very lragile; pieces easily break off at edges and cornors. ancl c "acks may develop inside the aerogel when handled, siuce its tensile strength is small. Near the edges. stresses caused by the mold may build up, which eventually lends to cracking. However, an internal "Frozen" sleess. ${ }^{.13 .12}$ which is formed in the gelation process due to an aligmment of finc chains of silicon. causes no cracling even over longer periods of time, since it is small. In on pplication craclis and broken fragments have little effect on performance. 
Machining aerogel is possible if done carefully. Except for lower-density material ( $\rho \leq 60 \mathrm{mg} / \mathrm{cm}^{3}$ ), aerogel can be held in a vise with appropriate cushioning. Smooth cuts can be obtained with a dianond blade running in a mill at $4200 \mathrm{rpm}$. However, it is important to wear a mask, since fine airborne $\mathrm{SiO}_{2}$ dust is generated. We have cut 1 inch thick pieces with a 4 inch blade. Cuts with diamond-covered wire provide a less smooth surface but may be more suitable for cutting thicker pieces. In order to catch the piece being cut, we hang a soft cotton towel behind the mill. Holes can be drilled witl an ordinary drill operated at $4200 \mathrm{rpm}$. Fine polish is obtained with Kleenex tissue or soft cotton. Machining of lowdensity material is much more difficult, because it is so fragile. A special vacuum chuch is required to hold the aerogel. Not much experience has been gathered so far. For our application, however, machining of low-density aerogel may not be an issue, since molds of any given shape can be made of glass, anodized aluminum or silicone.

Aerogel is affected by most fluids. Hydrophyllic aerogel exposed to water is destroyed immediately, since $\mathrm{H}_{2} \mathrm{O}$ molecules fill the pores. This results in a collapse of the aerogel strusture which can be rather explosive. Acetone and alcohol have similar destructive effects on any aerogel, turning it into a white powder immediately. Contact with oil results in visible surface damage. Exposure of aerogel to organic liquids and gases, even in small amounts, must be avoided, since it may cause structural changes, leading to a density increase or other damage. Therefore, outgassing of paints and wavelength shifters, which cover the walls of the aerogel containers, is an important issue. ${ }^{44}$ So far only fluid paper (green label) does not show visible surface effects. Detailed stuclies must be performed to find appropriate materials.
A erogel is quite fragile, but can be machined.

Aerogel is damaged by most gases and liquids. 
Figure 35.

Basic cell design for fluorescent fiber plus SPAD readout.

Two aerogel cells with diflerent refractive indices suffice to cover the entire momentum range.

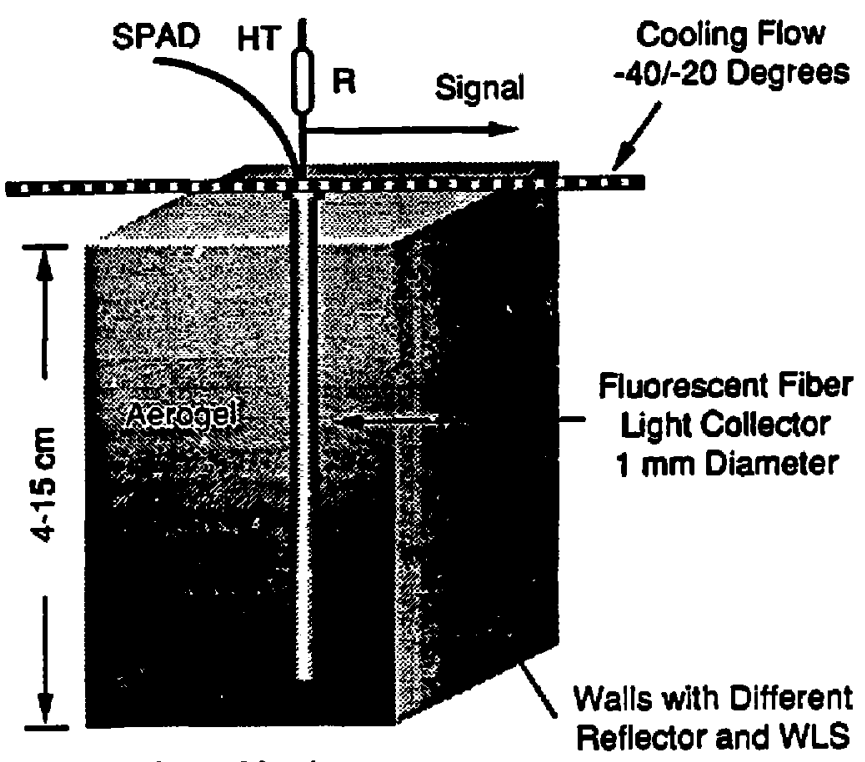

$2 \times 2 \mathrm{~cm}$, Matches

Crystal Cross Section

\section{Conceptual design of a Cell}

W e first describe a cell design similar to that in the PSI proposal. ${ }^{38,45}$ The principle cell layout is sketched in Figure 35. Small cells of aerogel are read out by a fluorescent fiber and an avalanche photodiode. Two cells, with refractive indices of $n=1.06$ and $n=1.008$, are necessary to cover the entire momentum range from 0.4 to $4.0 \mathrm{GeV} / \mathrm{c}$. Below $0.5 \mathrm{GeV} / \mathrm{c}, d E / d x$ is expected to provide good $K / \pi$ separation. In the forward region of the detector, cells with lower refractive indices may be used. For example, the refractive index $n=1.06$ may be lowered to $n=1.04$ to increase the proton threshold such that their observation is unlikely, while the refractive index $n=1.008$ may be lowered to $n=1.006$ to increase the kaon threshold up to $4.5 \mathrm{GeV}$. For mechanical reasons, however, $n=1.008$ is preferced over $n=1.006$. In Table 7, thresholds for pions, kaons and 
protons are listed in addition to the Cerenliov angles for different refractive indices under consideration.

Typical block sizes are $3 \times 3 \times 4 \mathrm{~cm}^{3}$ for $n=1.06$ and $2 \times 2 \times 10 \mathrm{~cm}^{3}$ for $n=1.008$. The blocks are appropriately tapered to provide projective geometry, matching the boundaries of the CsI crystals in the electromagnetic calorimeter. In order to optimize the light yield, the lower-density cell comes first, the higher-density cell second. Each block is housed in a light-tight, gas-tight, low-density container. This can be achieved with aluminized mylar, covered on one side either by black paint or black cardboard. The inner walls are sprayed with a highly reflecting white paint, which in addition may be coated with a thin layer of wavelength shifter (WLS), such as p-terphenyl, POPOP or PMP. The wall caating with WLS is most effective in a small-cell geometry, since here the probability for shifting UV light to higher wavelengths, where aerogel is highly transmissive, is larger than the probability for scattering and absorption. An alternative solution for a large-cell geometry is to mix a WLS into the aerogel. This idea wilf be discussed in more detail below.

\begin{tabular}{|c|c|c|c|c|}
\hline$n$ & $p_{\pi}[\mathrm{GeV} / \mathrm{c}]$ & $p_{K}[\mathrm{GeV} / \mathrm{c}]$ & $p_{p}[\mathrm{GeV} / \mathrm{c}]$ & $\theta_{c}$ \\
\hline 1.06 & 0.4 & 1.42 & 2.7 & $19.4^{\circ}$ \\
1.0173 & 0.75 & 2.65 & 5.03 & $10.6^{\circ}$ \\
1.026 & 0.6 & 2.15 & 3.9 & $12.9^{\circ}$ \\
1.008 & 1.1 & 3.9 & 7.4 & $7.2^{\circ}$ \\
\hline
\end{tabular}

To collect the photons from the aerogel and transport them to a photon detector, a thin fluorescent flux concentrator (FFC) of $1 \mathrm{~mm}$ diameter is inserted into a channel which is either drilled or made by the mold. The FFC is basically a transparent unclad plastic fiber which contains several different fluorescent laser dyes. The emission and absorption spectra of these laser dyes are matched such that light of lower wavelengths is shifted into a region where the photon detector has optimal quantum efficiency, Flux concentrations of a factor of 10-100 are achievable.
One design uses small cells of aerogel read out with a fiuorescent fiber coupled to a SPAD.

Table 7. Momentum thresholds and Cerenkov angles for different refractive indices. 
$S P A D$ 's require quenchisg.

Cooling to $-20^{\circ} \mathrm{C}$ reduces noise considerably.
For the photon detector, a single photon avalanche diode (SPAD) is used, matching the diameter of the fiber. For optimal coupling of the fiber to the SPAD, a customized holder is mounted to the SPAD after removing its window (see below). The fiber is inserted through a hole in the center, minimizing the distance between the photosensitive surface and the fiber. The SPAD, which works in a magnetic field, is operated in the Geiger mode, thus providing a high gain of $>1 \times 10^{8}$. The SPAD must be quencled using either an active or passive circuit, in order to prevent a continuous discharge of the photodiode. In passive quenching, the photodiode is normally kept in the non-conducting state by using a large load resistor $R_{L}$. An avalanche triggered by a photoelectron or bulk-generated electron in the depletion region discharges the pliotodiode from its reverse bias $V_{R}$ to a voltage slightly lower than the breakdown voltage $V_{B R}$. Recharging it back to the operating voltage $V_{R}-J_{d s} \cdot R_{L}$, where $I_{d s}$ is the surface datk current, has a time constant $C R_{L}$ of 1 ypically 300 ns where $C$ is the total capacitance including all stray capacitances. In active quenching a circuit drops the operating voltage below the breakdown voltage for a few 100 ns after an avalanche discharge. This allows for a collection of all remaining electrons and holes. When the operating voltage is reapplied, there are no electrons left in the depletion layer to trigger a new avalanche. Recharging through a small load capacitor or a transistor cau be very rapid. The advantages of active quenching ate shorter dead times and a synchronization of the detector with the beam crossing. A slight disadvantage is that the probability of after-pulsing is higher: $10 \%$ for 100 ns delay time compared to $0.6 \%$ for 300 ns.

SPADs are rather noisy. The noise rate is $10 \mathrm{kHz}$ at room tenperature. Cooling to $-20^{\circ} \mathrm{C}$ reduces the noise by a factor of 50 . Since a thermally generated electron may triggri an avalanche, noise levels have to be reasonably low. If the SPADs are gated for $50 \mathrm{~ns}$, one expects $5 \times 10^{-1}$ uoise counts at toom temperature. Thus, in a system of 20,000 cells, 10 randomly distributed SPADS will fire accidentally per event. This is certainly tolerable. Lower noise occupancies can be achieved by cooling 
the SPADs. However, this requires a cooling system and water-tight sealing of the cells.

In this design, the region around the fiber has to be discarded, because charged particles traversing the fiber or the SPAD produce Cerenkov photons at lower momenta due to larger refractive indices and thus create a signal. Although such particles can be located from the projected trajectory in the drift chamber, they cannot be identified. In order to reduce inefficiencies the diameters of the fibers and SPADs have to be small compared to the cell dimension.

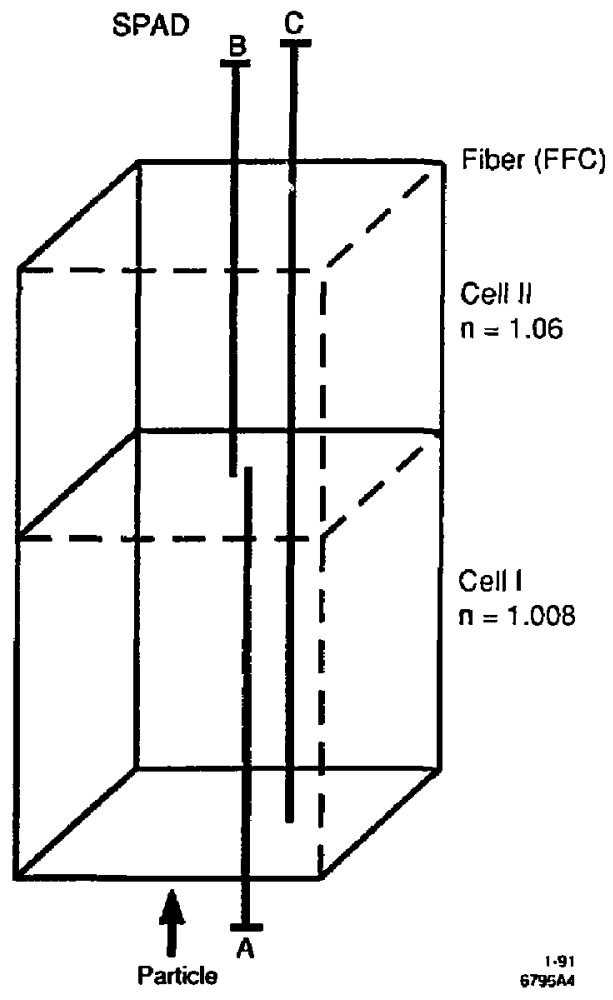

In order to obtain a very low noise performance without cooling and to keep the fiducial volume of the entire cell for most tracks, one can use two fibers per cell in
Figure 36. A two cell design for a readout with three fluorescent fibers. 
Unambiguous particle identification is provided by two cells of aerogel read out by three fibers. coincidence. Three rather than four fibers suffice for two cells, since tlie front cell and back cell can share one fiber, as shown in Pigure 36 . If we call the fiber in the lowerdensity cell $A$, the one in the higher-density cell $B$ and the fiber shared by both cells $C$, the following physical coincidence patterns car occur:

1. $A C, B C$ : a pion with $p_{\pi} \geq 1.1 \mathrm{GeV} / \mathrm{c}$ or a kaon with $p_{K} \geq 3.9 \mathrm{GeV} / \mathrm{c}$.

2. $\overline{A C}, B C$ : a pion with $0.4 \leq p_{\pi} \leq 1.1 \mathrm{GeV} / \mathrm{c}$ or a kaon with $p_{\kappa} \geq 1.4 \mathrm{GeV} / \mathrm{c}$.

3. $\overline{A C}, \overline{B C}$ : a pion with $p_{\pi} \leq 0.4 \mathrm{GeV} / \mathrm{c}$, a kaon with $p_{H} \leq 1.4 \mathrm{GeV} / \mathrm{c}$, or a proton with $p_{P} \leq$ $2.7 \mathrm{GeV} / \mathrm{c}$.

Sinct the particle trajectory and its momentum are known, the observation of a coincidence pattern together with the $d E / d x$ information provides identification of kaons and pions of all momenta inside the fiducial detector volume, while for $K / p, \pi / \epsilon$ and $\pi / \mu$ separation additional information is necessary.

Note that the coincidence $\mathrm{p}$ tern $A C, \overline{B C}$ is only observed if the track misses the front cell, SPAD $B$ does not five duc to an inefficiency, or SPAD $A$ and SPAD $C$ accidentally fire. Since the accidental rate is low $(1 \mathrm{~Hz}$ for 100 ns coincidence time), and a geometric miss can be detected, the observation of $A C, \overline{B C}$ provides a measure for inefficiencies. Since the momentum is known, this observed coincidence pattern may still be useful for particle ID: for $\mu>1.1 \mathrm{GeV} / \mathrm{c}, \mathrm{SPAD} B$ was inefficient and the particle was very likely a pion; for $0.4 \leq p \leq 1.1 \mathrm{GeV} / c$, SPAD $B$ l:as inefficient and SPAD $A$ was accidentally triggered. 'lo allow for unore redundancy one may use four filders where two are shared by both cells. If one again calls them $A, B, C$, and $D$, where $C$ and $D$ are the sluared fibers. then one can form four coincidences: $A C, A D . B C$ and $B D$, and analyze patterns simi. zr to those discussed abote.

An alteruative cell design consists of larger aerogel cells jeacl out by a phototetrode (PT), as shown in Figure 37. 'Typical cell sizes are $12 \times 12 \mathrm{~cm}^{2}$ for $n=1.008$ 


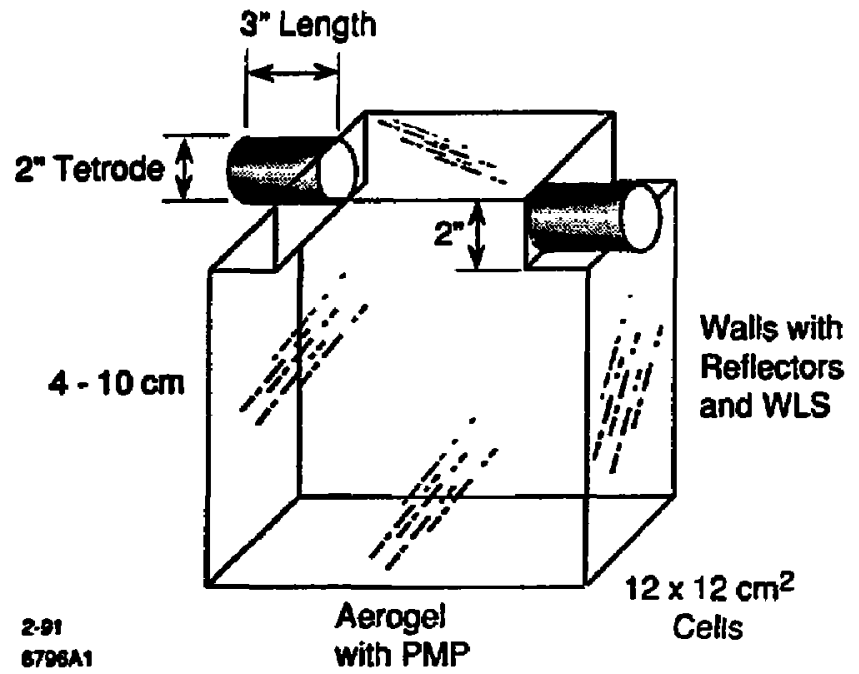

Figure 37. Basic cell design for phototetrode readout.

and $13.5 \times 13.5 \mathrm{~cm}^{2}$ for $n=1.06$. As before, the minimum cell heights are $4 \mathrm{~cm}$ and $10 \mathrm{~cm}$, but tine cells are extended to allow for optimal coupling of the aerogel to the PT. To provide projective geometry, the cells are appropriately tapered. In this arrangement, each aerogel cell covers the solid angle of nine CsI crystals. Crucial for this design is the use of aerogel mixed with WLS to achieve large scattering lengths. The containers housing the cells are also fabricated from white-painted aluminized mylar. Each cell is read out by two 2 inch PT's, which are oriented parallel to the magnetic field. An alternative design uses larger cells read out by vacuum phowotetrodes. The PT of the front and back cells are interleaved to save space. The PT's have a maximum have a gain of 50; further amplification is thus required. Though the expected signals are small, as shown in the next section, preamp noise is tolerable for this readout scheme. The equivalent noise charge of a preamplifier for this circuit is given by ${ }^{46}$ :

$$
\overline{E N C}^{2}=8 e_{n}^{2} \frac{1}{\lambda_{i}}\left[C_{d}+C_{F E T}\right]
$$

where:

$e_{n}$ : rms spot noise of the FET $(\mathrm{V} / \sqrt{\mathrm{Hz}})$

$C_{d}$ : capacitance of the delector $(<10 p \Gamma)$ 
Figure 38. Implementation of a two-cell aerogel threshold Cerenkov counter system into a general-purpose $B$ Factory detector.

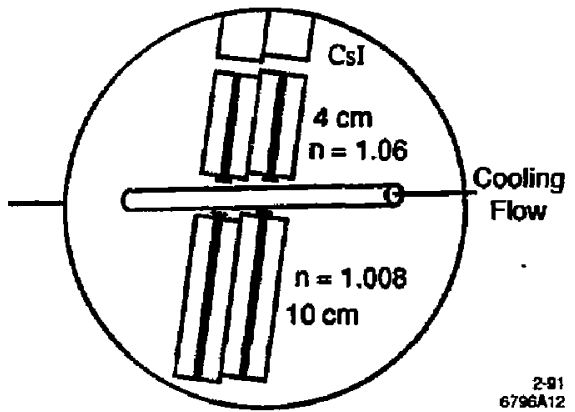

$C_{F E T}:$ capacitance of FET (on the order of $10 p F$ ) $\lambda_{t}: \quad$ integration time.

By integrating the pulses for a few microseconds, the ENC can be reduced to less than $30 \mathrm{rms}$ electrons, although this is state-of-the-art performance.

In order to keep the entire cell as fiducial volume, both PT must be put in coincidence. While the smallcell design with the FFC/SPAD readout provides high granularity, so that the probability of two tracks in an event hitting the same cell is very small, the large-cell design with PT readout reduces the number of channels by a factor of $>10$. Figure 38 shows the implementation of aerogel threshold Cerenkov counters into the B Factory multipurpose detector. The magnified view shows the arrangement of the two cells in the presence of a cooling line.

\subsection{Light Yield and Efficiency}

A charged particle with a velocity $\beta$ traversing a medium of refractive index $n$ radiates Cerenkov photons under the angle $\theta_{c}=\cos ^{-1}(1 /(n \beta))$, if $\beta \geq 1 / n$. Assuming that the cell height $L$ is much larger than the wavelength $\lambda(\lambda \ll L)$, the number of Cerenkov photons radiated per wavelength is:

$$
d N=\frac{2 \pi \alpha}{\lambda^{2}} L \sin ^{2} \theta_{c} d \lambda,
$$

where $\alpha$ denotes the fine structure constant. Integrating $\mathrm{Eq}$. (7.2) over $\lambda$ from 220 to $550 \mathrm{~nm}$ yields for a. $1 \mathrm{~cm}$ thick block: 


$$
N=1.25 \times 10^{3} \sin ^{2} \theta_{c} \text { photons } .
$$

An increase of the sensitivity range to $200-5801 \mathrm{~m}$ gives a $20 \%$ higher yield of Čerentiov photons.

We will first discuss the light collection process in the context of the FFC/SPAD-readout scheme. The Cerenkov photons have to undergo a complex process to reach the SPAD and trigger an avalanche. The plston path, consisting of several bounces off the container walls, is altered by several Rayleigh scatterings in the aerogel. Typical values for different cell designs are given in the next section. For cell designs under consideration, the number of reflections is about 15 . Before reaching the detector, the main losses are due to absorption in the aerogel and absorption on the walls. A photon reaching the fiber may be absorbed and emitted at a different angle with a higher wavelength. If $\sin \theta<1 / n_{f i b}$ the photon is trapped in the fiber. Since the fiber contains several laser dyes, the photon may be reabsorbed and reemitted at a different angle and a higher wavelength. If the new angle does not satisfy the condition for total reflection, the photon might leave the fiber and eventually be absorbed in the aerogel. Several dyes are necessary to shift the photon to $800 \mathrm{~nm}$, whece the sensitivity of the SPAD is largest. Upon reaching the SPAD, the photon must create an electron-hole pair, which has to trigger an avalanche. Considering all these effects, the expected number of photoelectrons for the FFC/SPAD readout is given by:

$N_{p . e .}=N_{p h} \cdot T_{A} \cdot R_{W}^{\bar{n}_{\tau}} \cdot Q_{W V} \cdot A_{F F C} \cdot Q_{F F C} \cdot T_{F F C} \cdot Q_{F-D} \cdot T_{D} \cdot Q_{D}$

where:

$N_{p h}: \quad$ produced number of Ćerenkov photons

$T_{A}: \quad$ transmission in aerogel: $1-\exp \left(-\bar{n} \bar{v} \mu_{a}\right) \sim 0.45$

$\bar{n}_{r}: \quad$ average number of bounces at the wall $(\sim 15)$

$\bar{w}: \quad$ average path length between bounces

$\mu_{\mathrm{a}}: \quad$ absorption coefficient, $10^{-2} \mathrm{~cm}^{-1}$

$R_{W}$ : reflectivity of the walls,$>0.95$ for $\lambda<300 \mathrm{~nm}$

and $>0.98$ for $300<\lambda<700 \mathrm{~nm}$ ) 
The overall photon detection efficiency is estimated to be a few percent.

Table 8. Number of Cerenkov photons and photoelectron yield for different refractive indices, using the calculated efficiency of a $2 \times$ $2 \mathrm{~cm}^{2}$ cell.
$Q_{W}: \quad$ quantum efficiency of WLS on the walls (0.9)

$A_{F F C}$ : photon absorption probability in the fiber (0.9)

$Q_{F F C:}$ quantum efficiency of dyes in the fiber $(0.9)$

$T_{F F C}$ : transmission efficiency in FFC including selfabsorption (0.2)

$Q_{F-D}: \quad$ coupling efficiency of FFC and SPAD (0.25)

$Q_{D}: \quad$ quantum efficiency of SPAD $(0.65)$

$T_{D}$ : efficiency for a photoelectron to trigger an avalanche $(0.6)$

For a $2 \times 2 \mathrm{~cm}^{2}$ cell, the efficiency is estimated to be $\epsilon=1.9 \%$, while for the $3 \times 3 \mathrm{~cm}^{2}$ cell, it is $\epsilon=1.3 \%$. Using these estimates, Table 8 shows the number of produced photons and the photoelectron yield for different refractive indices computed for $220<\lambda<550 \mathrm{~nm}$ and $200<\lambda<580 \mathrm{~nm}$.

For the alternative design of large aerogel cells with PT readout, the photoelectron yield is determined by:

$$
N_{p . e .}=N_{p h} \cdot T_{A} \cdot R_{W}^{\bar{n}_{r}} \cdot T_{\text {tet }} \cdot Q_{\text {tet }}
$$

where:

$T_{\text {tet }}$ : transmission coefficient of the PT window (0.95) $Q_{\text {tet }}$ : quantum efficiency of the PT photocathode (0.2) For a $15 \times 15 \mathrm{~cm}^{2}$ cell, the transmission coefficient $T_{A}$ is only 0.1 , while the average number of reflections at the walls is 10 . The efficiency is estimated to be $\epsilon=1.6 \%$, which is comparable to the efficiency of the FFC/SPAD readout. The yield of Cerenkov photons and photoelectrons estimated for a $2 \times 2 \mathrm{~cm}^{2}$ for different refractive indices is summarized in Table 8 .

\begin{tabular}{|c|c|c|c|c|}
\hline $\mathrm{n}$ & $\mathrm{L}[\mathrm{cm}]$ & $N_{p h}^{\dagger}$ & $N_{\text {p.e. }}^{J \dagger}$ & $N_{p . e .}^{I 1 \dagger}$ \\
\hline 1.06 & 4.0 & $552(663)$ & $10.5(12.6)$ & $8.8(10.6)$ \\
1.0173 & 10.0 & $423(508)$ & $8.0(9.7)$ & $6.8(8.1)$ \\
1.026 & 4.0 & $250(300)$ & $4.8(5.7)$ & $4.0(4.8)$ \\
1.008 & 10.0 & $196(235)$ & $3.7(4.5)$ & $3.1(3.8)$ \\
\hline
\end{tabular}

$\dagger$ The first set of numbers represent yields for the interval $220 \mathrm{~nm} \leq$ $\lambda \leq 550 \mathrm{~nm}$, the second sel for the interval $200 \mathrm{~nm} \leq \lambda \leq 580 \mathrm{~nm}$. 


\subsection{Monte Carlo Simulations}

In order to obtain a realistic estimate of all factors entering Eqs. (7) and (8), it is necessary to study the photon paths in different cell designs in more detail. For this purpose a Monte Carlo program has been developed using vector algorithms. Cerenkov photons are generated uniformly in $x, y, z$ inside a rectangular box. Assuming that the trajectory of the charged track is parallel to the $z$ direction, which in turn is parallel to the cell height, the direction of the Cerenkov photons is generated isotropically in $\phi$ and $\cos \theta$, with $\cos \theta$ limited to the interval $\left[1, \cos \theta_{c}=1 /(\beta n)\right]$. In addition, a wavelength is generated for each photon according to the Cerenkov spectral distribution $d N / d \lambda \propto 1 / \lambda^{2}$. Next the path length is determined, considering diffuse reflection on the walls with wavelength-dependent absorption. Both Rayleigh scattering and wavelength-dependent absorption in the aerogel are considered. The scattered photon is generated with the correct angular distribution. If the photon hits the fiber or the PT, it is recorded as a hit. In the future, it is planned to include wavelength shifting in the aerogel, wavelength shifting on the walls plus a detailed simulation of the transport of photons through the fiber. Furthermore, the simulation of different geometric shapes, such as trapezoids and pyramids will be made avajlable.

With the current Monte Carlo model, samples of 10,000 events have been generated both for the FFC/SPADreadout scheme and the $\mathrm{PT}$-readout scheme for different cell sizes. ${ }^{47}$ The index of refraction has been set to $n=1.06$. Figures 39 and 40 show the resulting distributions for the number of reflections $n_{r}$, number of Rayleigh scatterings $n_{s c}$, total path length $l_{y}$, and the path length between reflections $l_{g} / n$ for a $2 \times 2 \times 10 \mathrm{~cm}^{3}$ cell, read out with a $1 \mathrm{~mm}$ fiber, and a $15 \times 1.5 \times 15 \mathrm{~cm}^{3}$ cell, read out with a $\mathrm{PT}$, respectively. The average values for these distributions are given in Table 9 together with the corresponding averages for other geometric arrangements. Figure 41 displays the average values for $\bar{n}_{r}, \bar{n}_{s c}, \bar{l}_{g}$, and $\overline{l_{g} / n_{r}}$ as a function of the cell width, $d$,
The Monte Carlo simulation includes the Cerenkov spectrum, Rayleigh scattering and wavelength-dependent absorption and wall reflection. 

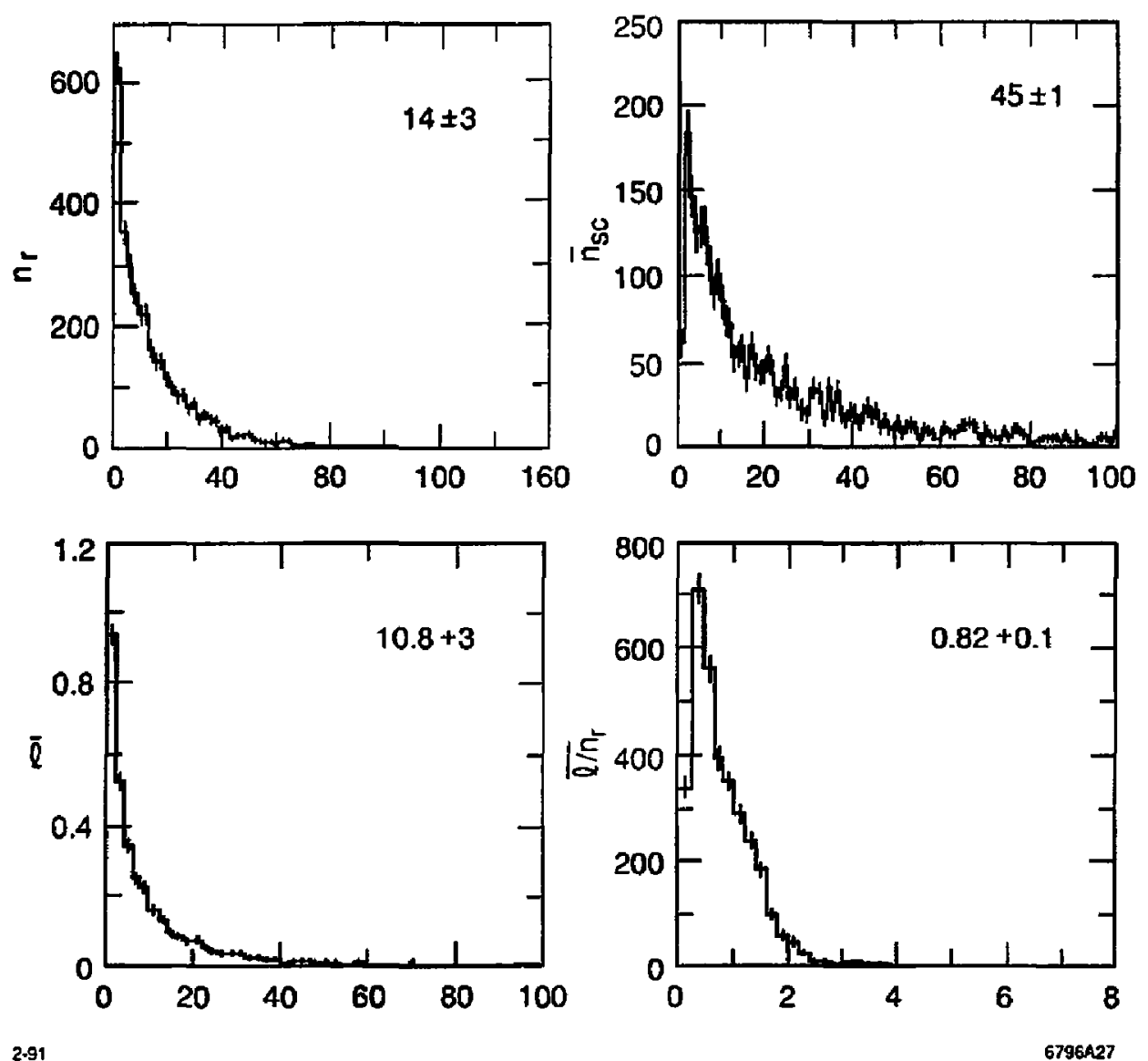

Figure 39. Monte Carlo distributions for a $2 \times 2 \times$ $10 \mathrm{~cm}^{3}$ cell with FFC/SPAD readout: (a) number of renections, (b) number of Rayleigh scatterings, (c) total path length and (d) path length per number of reflectjons.

The efficiency depends strongly" on the transverse cell size and weakly on the cell length. for cell heights, $h$, of $4 \mathrm{~cm}$ and $10 \mathrm{~cm}$ in the FFC/SPADreadout scheme and for $15 \mathrm{~cm}$ long cells in the PT. readout scheme. For the FFC/SPAD-readout scheme, $\bar{n}_{r}$ first incleases rapidly with $d$ because of increasing path lengths. At about $d=4 \mathrm{~cm}$, the path lengths are so large that due to absorption, the tails of the $n_{r}$ distribution are truncated and thus $\bar{n}_{r}$ starts to fall. Since the fiber is parallel to the cell height, the dependence of $\bar{n}_{r}$ on $d$ is weak; for example the maximum $\bar{n}_{r}$ is 16 fol $d=4 \mathrm{~cm}$ compared to $\bar{n}_{r}=14$ for $d=10 \mathrm{~cm}$, being slightly larger for shorter cells because average path 

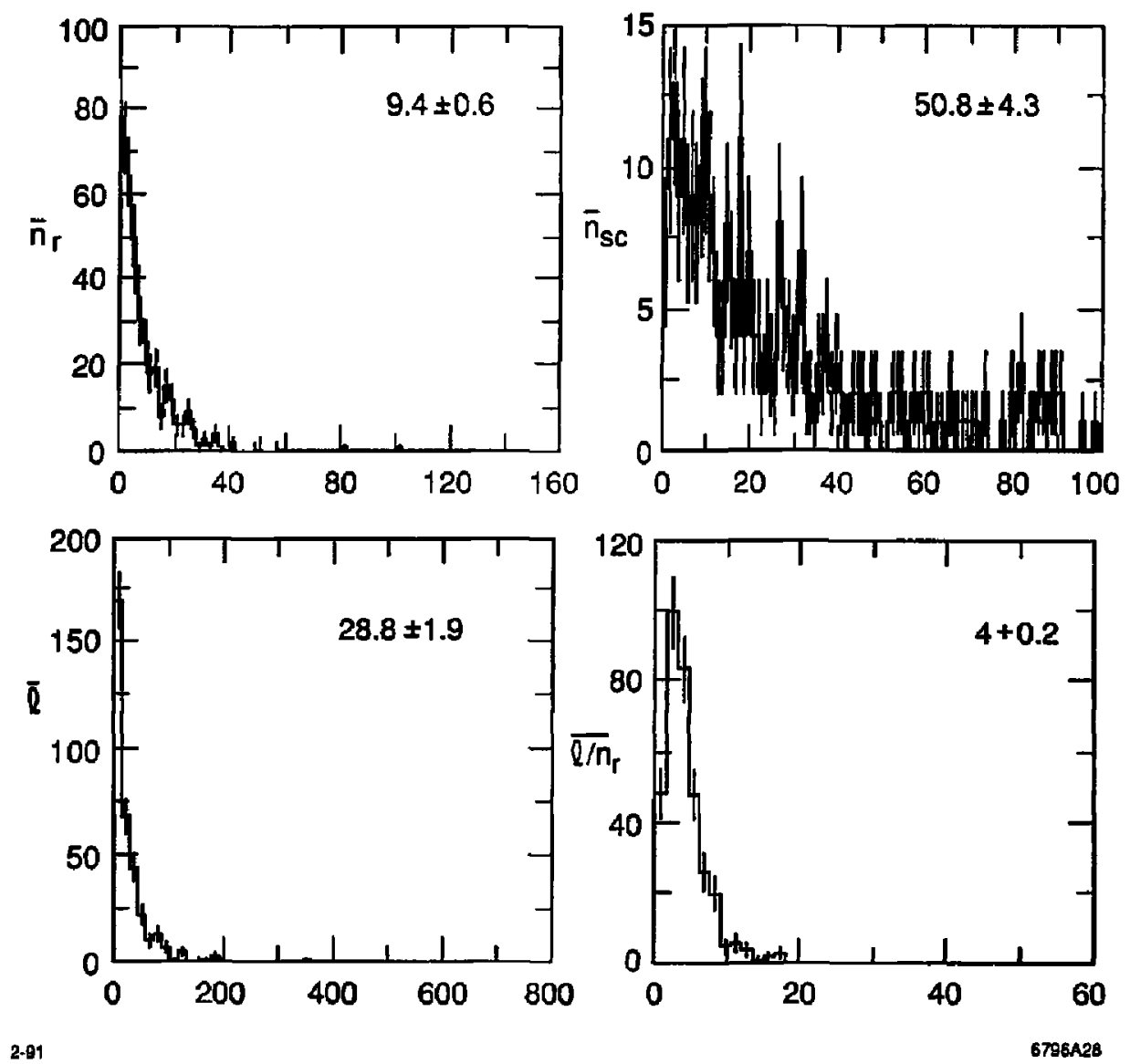

lengths between reflections are smaller and thus tails in the $n_{r}$ distribution are larger. The average number of Rayleigh scatterings increases rapidly with $d$ due to an increase in $\bar{l}_{g}$, whereas $\bar{n}_{s c}$ is basically inclependent of $h$. The average length between reflections rises slowly with $d$ and is obviously larger for long cells than lor short cells. The efficiency decreases rapidly with $d$, as expected, but is independent of $h$. Since for the PT-reaclout scheme the average path length is already rather large, tails of the $n_{r}$ and $n_{g c}$ distributions are truncated, thus yielding lower averages. Larger cell sizes do not alter this fact, such

Figure 40. Monte Carlo distributions for a $15 \times 15$ $\times 15 \mathrm{~cm}^{3}$ cell a 2 inch phototetrode readout: (a) number of reflections, (b) number of Rayleigh scatterings, (c) total path length and (d) path length per number of reflections. 
that $\bar{n}_{r}$ is 10 independent of $d$ and $n_{s c}$ rises only slowly with $d$. The variable $\overline{I_{y} / n_{r}}$ is about a factor of two larger than for the FFC/SPAD-readout scheme. The efficiency is rather low, but is about a factor of two larger than for sinnilat cell sizes in the FFC/SPAD-readout design.

\begin{tabular}{|c|c|c|c|c|c|c|}
\hline $\mathrm{d}[\mathrm{cm}]$ & $\mathrm{h}[\mathrm{cm}]$ & $\tilde{n}_{r}$ & $\bar{n}_{s c}$ & $\bar{l}_{g}[\mathrm{~cm}]$ & $\overline{l_{g} / n_{r}}[\mathrm{~cm}]$ & $\epsilon \%$ \\
\hline 0.5 & 4.0 & $7.5 \pm 0.1$ & $8.3 \pm 0.1$ & $2.32 \pm 0.03$ & $0.366 \pm 0.004$ & $77.9 \pm 0.9$ \\
1.0 & 4.0 & $12.4 \pm 0.2$ & $21.9 \pm 0.4$ & $5.6 \pm 0.09$ & $0.58 \pm 0.005$ & $58.1 \pm 0.5$ \\
1.5 & 4.0 & $15.2 \pm 0.3$ & $34.9 \pm 0.8$ & $8.68 \pm 0.17$ & $0.62 \pm 0.007$ & $43.4 \pm 0.7$ \\
2.0 & 4.0 & $16.3 \pm 0.3$ & $44.0 \pm 1.2$ & $11.0 \pm 0.3$ & $0.72 \pm 0.01$ & $32.7 \pm 0.6$ \\
3.0 & 4.0 & $16.9 \pm 0.4$ & $54.2 \pm 1.8$ & $14.8 \pm 0.5$ & $0.9 \pm 0.01$ & $21.4 \pm 0.5$ \\
4.0 & 4.0 & $16.0 \pm 0.5$ & $61.0 \pm 2 . \overline{1}$ & $16.3 \pm 0.5$ & $1.02 \pm 0.02$ & $13.4 \pm 0.4$ \\
5.0 & 4.0 & $15.8 \pm 0.6$ & $57.3 \pm 2.6$ & $19.5 \pm 1.0$ & $1.2 \pm 0.03$ & $10.4 \pm 0.3$ \\
10.0 & 4.0 & $11.7 \pm 0.9$ & $71.7 \pm 6.1$ & $19.2 \pm 2.0$ & $3.4 \pm 0.03$ & $3.3 \pm 0.2$ \\
\hline 0.5 & 10.0 & $6.9 \pm 0.1$ & $8.5 \pm 0.1$ & $2.3 \pm 0.03$ & $0.395 \pm 0.004$ & $79.2 \pm 0.9$ \\
1.0 & 10.0 & $11.5 \pm 0.2$ & $23.6 \pm 0.5$ & $5.5 \pm 0.09$ & $0.54 \pm 0.006$ & $58.3 \pm 0.8$ \\
1.5 & 10.0 & $13.8 \pm 0.2$ & $34.2 \pm 0.8$ & $9.0 \pm 0.2$ & $0.70 \pm 0.009$ & $44.0 \pm 0.7$ \\
2.0 & 10.0 & $14.1 \pm 0.3$ & $45.0 \pm 1.2$ & $10.8 \pm 0.3$ & $0.82 \pm 0.01$ & $33.8 \pm 0.6$ \\
3.0 & 10.0 & $13.8 \pm 0.4$ & $53.3 \pm] .7$ & $14.1 \pm 0.5$ & $0.99 \pm 0.02$ & $21.4 \pm 0.5$ \\
4.0 & 10.0 & $13.7 \pm 0.5$ & $62.4 \pm 2.6$ & $16.1 \pm 0.7$ & $1.16 \pm 0.03$ & $14.6 \pm 0.4$ \\
5.0 & 10.0 & $13.1 \pm 0.5$ & $59.3 \pm 3.0$ & $19.2 \pm 0.9$ & $1.4 \pm 0.06$ & $11.1 \pm 0.3$ \\
10.0 & 10.0 & $10.1 \pm 0.7$ & $67.2 \pm 5.2$ & $20.1 \pm 1.8$ & $1.8 \pm 0.08$ & $3.3 \pm 0.2$ \\
\hline 5.0 & 15.0 & $9.9 \pm 0.2$ & $39.2 \pm 2.3$ & $18.2 \pm 0.5$ & $2.7 \pm 0.05$ & $18.5 \pm 0.4$ \\
10.0 & 15.0 & $9.7 \pm 0.2$ & $40.5 \pm 1.5$ & $27.0 \pm 1.0$ & $3.6 \pm 0.09$ & $7.7 \pm 0.3$ \\
15.0 & 15.0 & $9.4 \pm 0.2$ & $50.8 \pm 2.0$ & $28.8 \pm 1.9$ & $4.0 \pm 0.2$ & $3.5 \pm 0.2$ \\
\hline
\end{tabular}

Table 9. Monte Carlo results.
For both designs, the $\left(1+\cos ^{2} \theta\right)$ dependence of Rayleigh scattering, the simulation of the Cerenkov angle and at variation of the index of refraction have no effect on the quantities discussed above. The inclusion of wavelenglli-dependent scattering and absorption in the aerogel and absorption on the walls are rather important. For example, if these effects are neglected in the FFC/SPAD-readout scheme, $\vec{n}_{r}$ increases by about a factor of two and $\bar{l}_{g}$ by about a factor of 4 . 

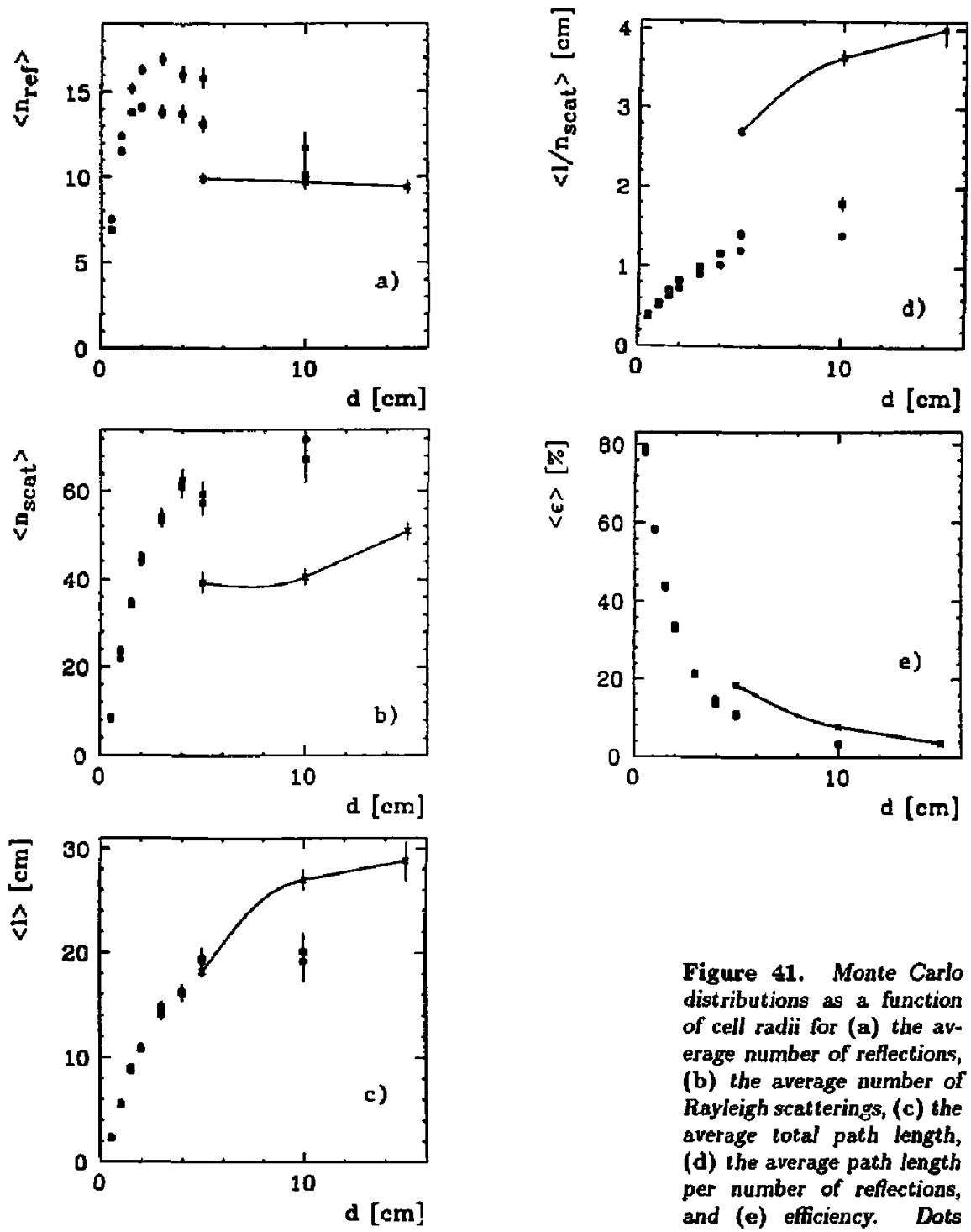

In conclusion, these studies show that the optimal cell size for the FFC/SPAD-readout scheme lies between 2 and $3 \mathrm{~cm}$, while for the PT-readout design $d$ should not be larger than $10 \mathrm{~cm}$.

Figure 41. Monte Carlo distributions as a function of cell radii for (a) the average number of reflections, (b) the average number of Rayleigh scatterings, (c) the average total path length, (d) the average path length per number of refiections, and (e) efficiency. Dots and squares show $4 \mathrm{~cm}$ and $10 \mathrm{~cm}$ long cells read out by a $1 \mathrm{~mm}$ fiber, while stars show $15 \mathrm{~cm}$ long cells read out with a 2 inch PT. 
The transmission of aerogel is well described by $a \lambda^{-4}$ scattering term and $a \lambda^{-2}$ absorption term.

Figure 42. Transmission of different aerogel samples from Aerglas.

\subsection{Preliminary Test Results}

Our first aerogel studies consisted of transmission measurements with an HP spectrophotometer, in order to determine the absorption length $\Lambda_{a}$ and the scattering length $\Lambda_{s}$. For a successful operation of aerogel counters, it is important to have large absorption lengths $\left(\Lambda_{u}\right\rangle$ $40 \mathrm{~cm}$ ). Figure 42 shows the transmittance measured for three samples manufactured by Aerglas: a $1.8 \mathrm{~mm}$ thick piece with $n=1.032$, which has been cul off a $12 \times 12 \times 2.5 \mathrm{~cm}^{3}$ thick block (several years old) and two recently made $3.0 \mathrm{~cm}$ thick pieces with $n=1.025$ and $n=1.055$. The measured curves can be fitted with an exponential function:

$$
T=T_{0} e^{-\mu(\lambda) d},
$$

where $d$ is the thickness, $T_{0}$ is the maximal transmission accounting for reflection and $\mu(\lambda)$ is the attenuation coefficient, which can be parametrized by a Rayleigh scattering term and an absorption term:

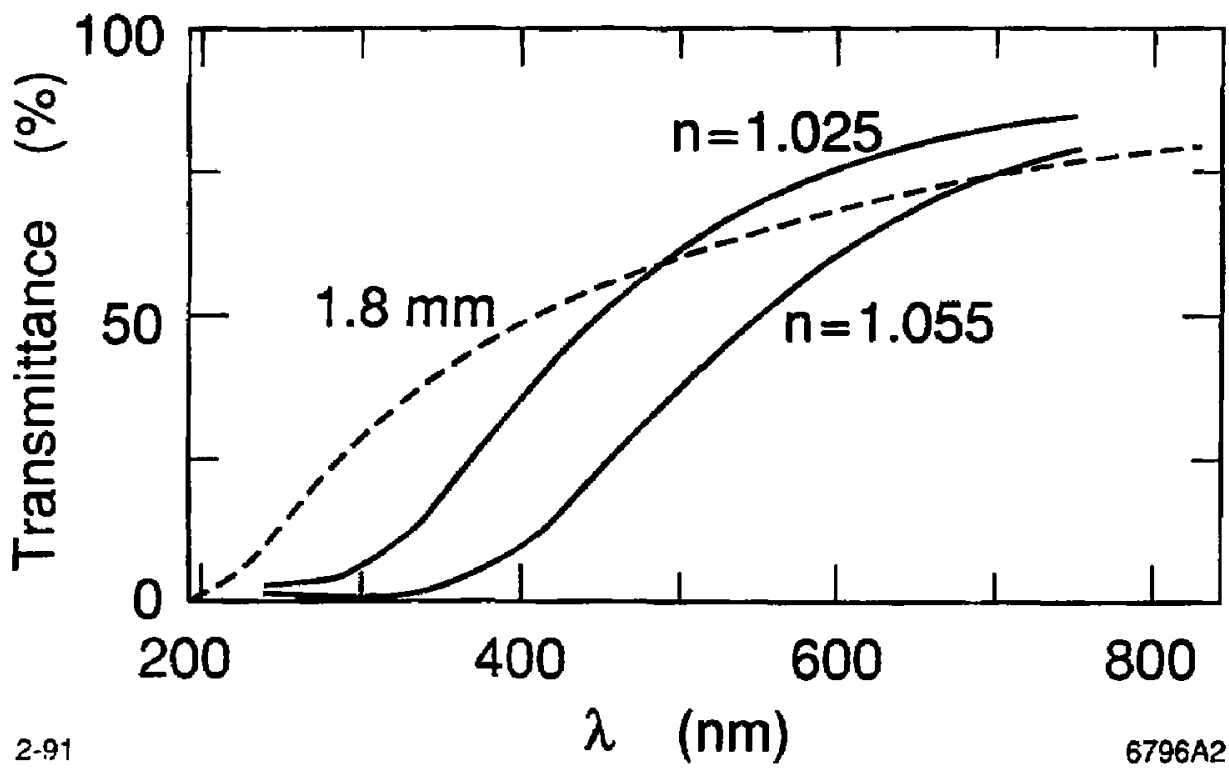




$$
\mu(\lambda)=a / \lambda^{4}+b / \lambda^{2}
$$

The average scattering and absorption lengths for Cerenkov distributed light are given by:

$$
\Lambda_{s}=\frac{1}{3 a} \frac{\left(\lambda_{\max }^{3}-\lambda_{\min }^{3}\right) \lambda_{\max } \lambda_{\min }}{\lambda_{\max }-\lambda_{\min }}
$$

and

$$
\Lambda_{a}=\frac{1}{b} \lambda_{\max } \lambda_{\min } .
$$

where $\lambda_{\max }$ and $\lambda_{\min }$ are the maximum and minimum wavelengths detected by the counter.

For technical reasons, it is more convenient to determine the measured values for $\mu(\lambda)$ first and then fit these with the parametrization in Eq. (7.7). The results are listed in Table 10. For comparison, measurement from other groups are also shown. Except for our aged samples, all other data are consistent. We lave done several measurements with samples from the aged block, which yielded similar results. The most plausible explanation is an aging effect, as the aerogel nay absorb water and gases from the air, thus changing its properties. Since similar effects have also been noticed by S. Schindler, ${ }^{44}$ this implies that proper storage is absolutely necessary. In addition, the aerogel in a detector cell must be well isolated from humidity and a careful choice for the reflector must be made. Thus, for a wellhandled sample of aerogel, the average scattering length is $\sim 3 \mathrm{~cm}$, while the average absorption length is $\sim 50 \mathrm{~cm}$ for the interval $300 \leq \lambda \leq 800 \mathrm{~nm}$.

It is important to store and use aerogel under airtight conditions.

Table 10. Absorption and scattering length in aerogel.

\begin{tabular}{|l|c|c|c|c|c|}
\hline \multicolumn{1}{|c|}{ Sample } & Thickness $[\mathrm{cm}]$ & $n$ & $\Lambda_{a}[\mathrm{~cm}]$ & $\Lambda_{s}[\mathrm{~cm}]$ & Sensitivity Range \\
\hline $\mathrm{CIT}$ & 0.18 & 1.032 & 0.4 & 3.6 & $200-800$ \\
$\mathrm{CIT}^{44}$ & 3.0 & 1.055 & 50.0 & 3.2 & $300-800$ \\
$\mathrm{CIT}^{44}$ & 3.0 & 1.025 & 46.0 & 6.2 & $300-800$ \\
Bonn $^{48}$ & 2.8 & 1.035 & 33.9 & 2.3 & $300-600$ \\
Novosibirsk $^{49}$ & 2.5 & 1.035 & $>100^{\dagger}$ & $2-3^{\dagger}$ & - \\
\hline
\end{tabular}

$\dagger$ Estimated for pure $\mathrm{SiO}_{2}$ aerogel. 


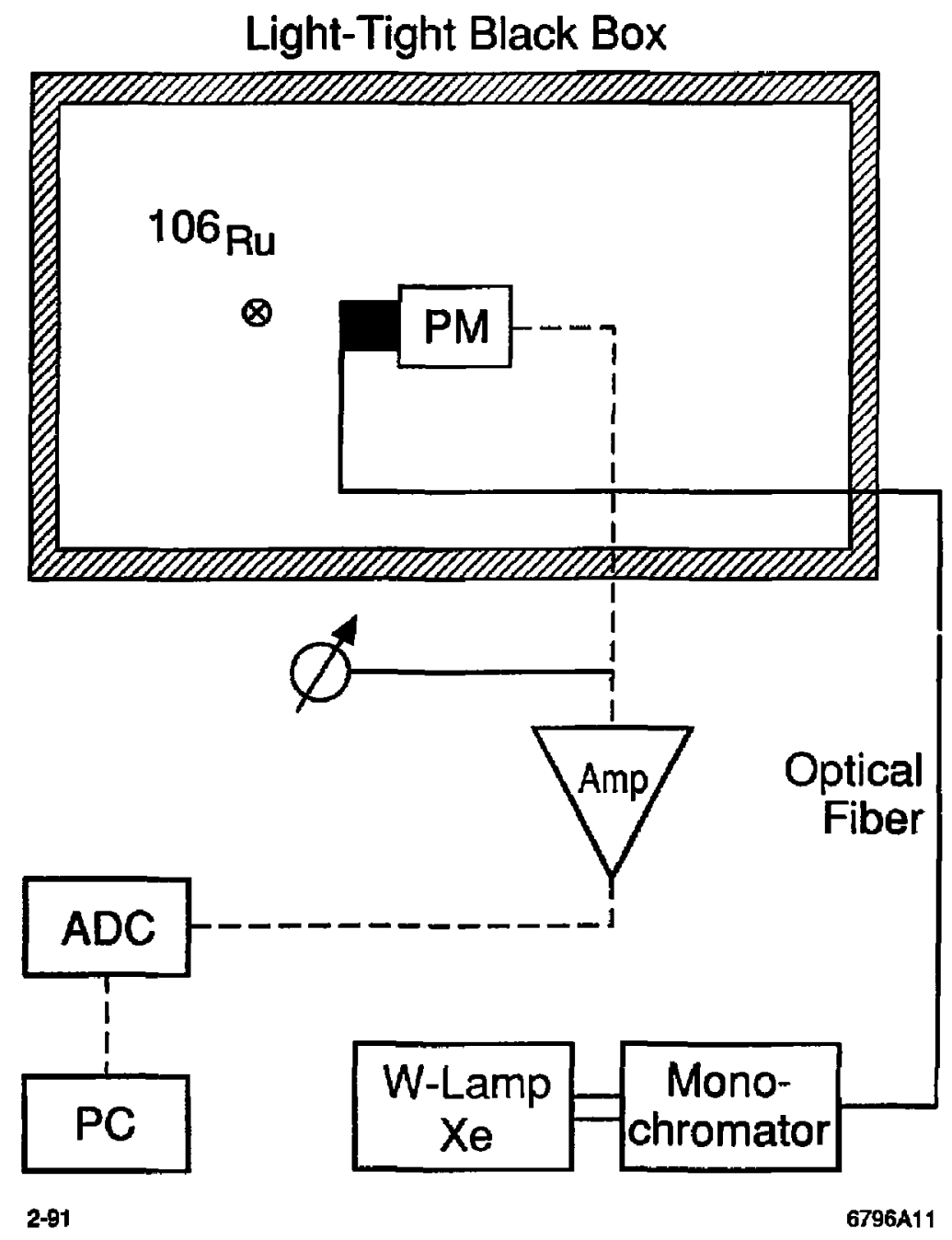

Figure 43. Test setup for measuring the efficiency from a light source as function of wavelength.
In order to study the properties of individual aerogel cells and monitor their performance the test setup shown in Figuce 43 was used. Pieces of aerogel housed in light-tight containers are attached to a photomultiplier, which is mounted in a black light-tight box. The container's are made of a $250 \mu \mathrm{m}$ thick layer of black card- 


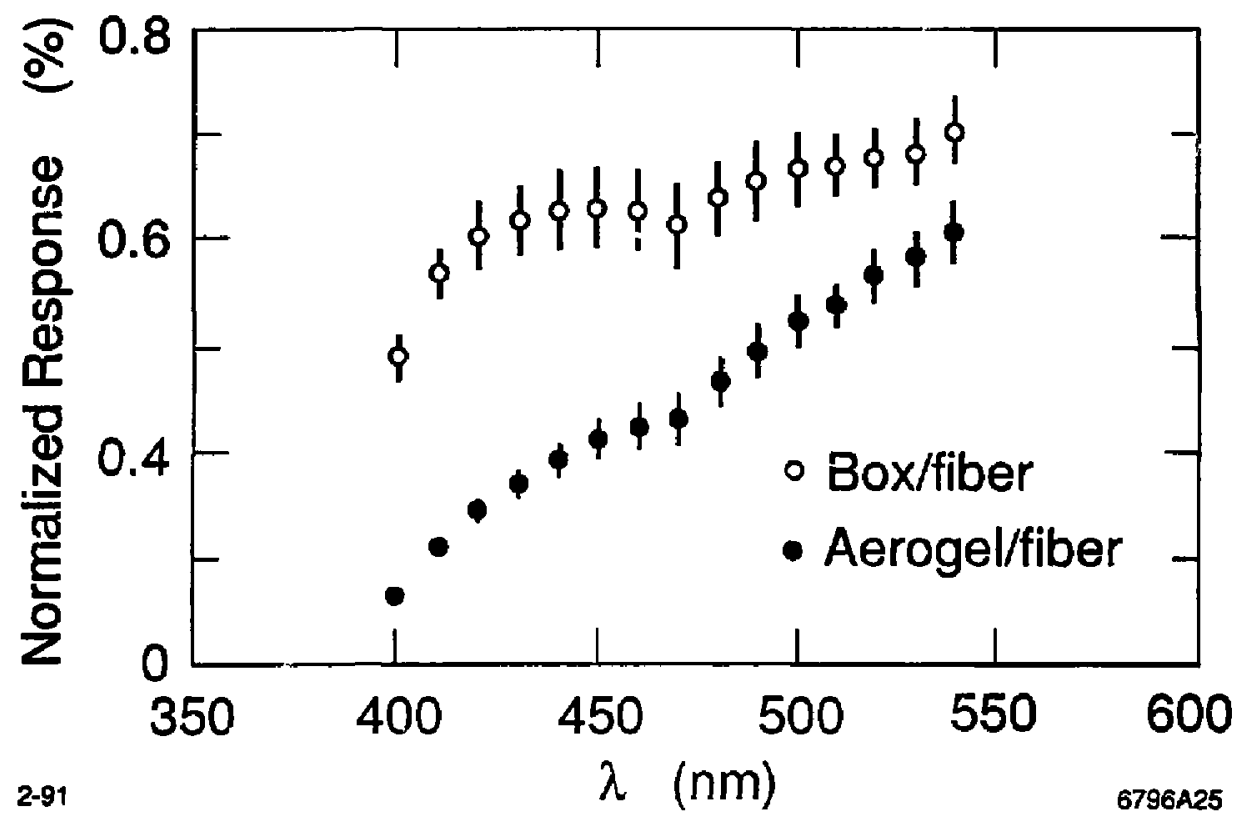

board glued to a $50 \mu \mathrm{m}$ thicl: layer of aluminized mylar, which is sprayed with highly-reflecting white paint (Nuclear Enterprise NE 560). For our current studies, light from either a tungsten or xenon lamp or electrons from the $\beta$ decay of ${ }^{106} \mathrm{Ru}$ was used. The light, after passing through a monochrometer, was fed via a clear plastic fiber into the cell, in such a way that the direct beam could not hit the photocathode. For a selected wavelength interval, the anode current was recorded with a digital ammeter. For the measurements with the ${ }^{106} \mathrm{Ru}$ source, the anode signal was amplified (Ortec EG\&G 560) and then passed into a peak-sensitive $A D C$ (Ortec EG\&G 916) operated by a PC.

Figure 44 displays the efficiency of a $3 \times 3 \times 4 \mathrm{~cm}^{3}$ block of aeroge! cell for visible light as a function of the wavelength. For comparison, the efficiency for the reflecting box itself is also shown. For normalization the intensity of the light beam is directly measured wit il
Figure 44. Efficiency of a $3 \times 3 \times 4 \quad n^{3}$ aerogel cell in the vi ble-light region. Solid dots show the data for the cell with aerogel, light dots show the data for the container itself.
A refectivity of $97 \%$ has been measured for NE560 paint in the visible region. 
Figure 45. Test setup for measuring the effciency and photoelectron yield of Cerenkov photons.

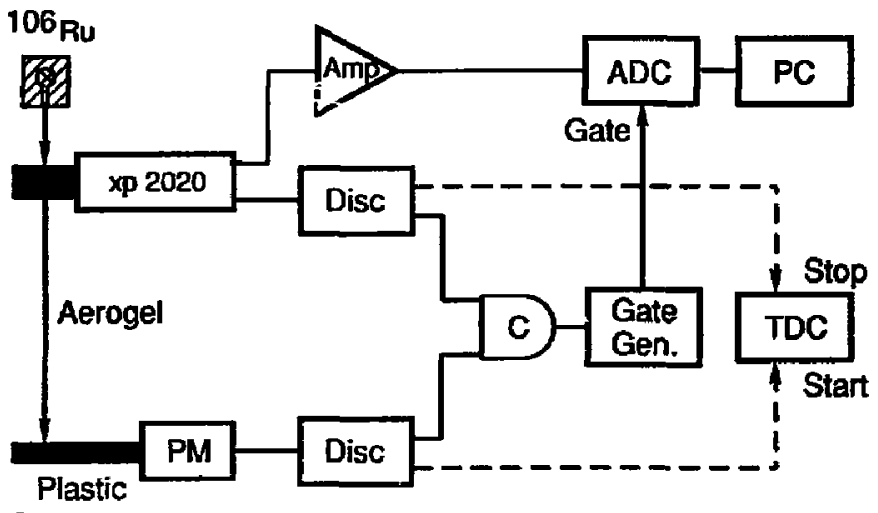

Scintillator

67909

the PM. The data are averages of three measurements; the errors are given by the corresponding standard deviations. In order to avoid saturation, measurements were performed at moderate high voltage and the light intensity was reduced appropriately. The data for the box without aelogel give the efficiency after $\bar{n}_{r}$ reflections, where for this geometry $\bar{n}_{r}$ is about 14. Above $420 \mathrm{~nm}$, the efficjency depenc's rather weakly on $\lambda$, averaging $\sim 65 \%$. This implies a reflectivity of $\sim 97 \%$. However, for the UV region, the reflectivity is significantly lower, requiring improvement. The properties of different diffuse reflecting materials are discussed in the next section. We require a reflectivity of $\geq 95 \%$ at $250 \mathrm{~nm}$, increasing to $98 \%$ above $350 \mathrm{~nm}$. The results for the aerogel cell show a strong wavelength dependence, as expected, due to Rayleigh scattering and absorption. These measurements will be extended to the UV-light region and visible red-light region. This technique can be used to study the dependence on geometric shapes, the eflects of wavelength shifters mixed into the aerogel, and to monitor the aging of aerogel.

The test setup shown in Figure 45 is used to measure the photoelectron yield from Cerenkov light in different aerogel cells. Electrons from a collimated ${ }^{106} \mathrm{Ru}$ source 


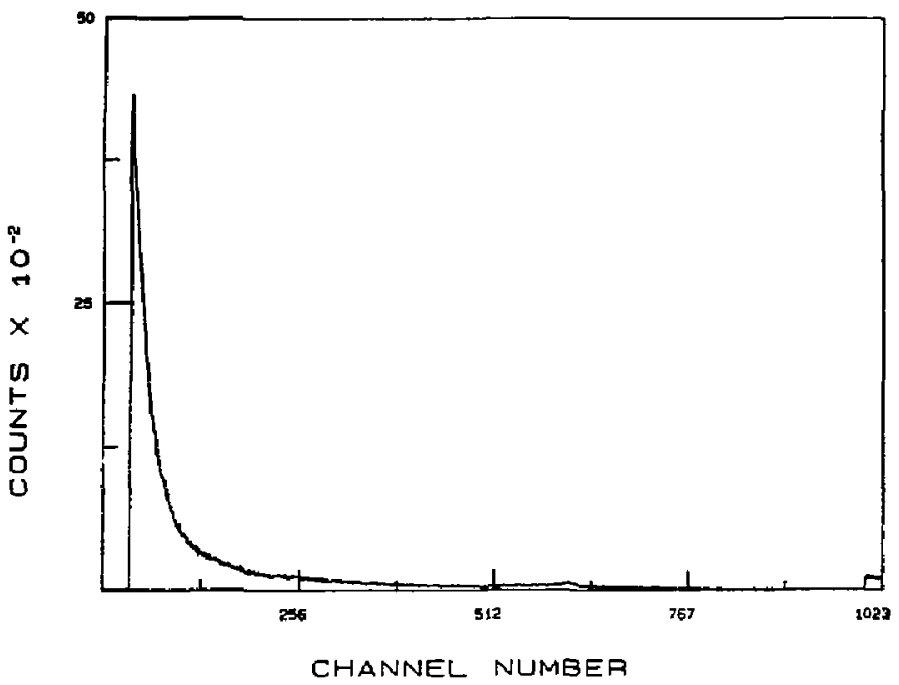

Figure 46. Cerenkov photon spectrum observed with a ${ }^{106}$ Ru source.

hit an aerogel cel] perpendicular to the photomultiplier. To trigger, the electron must penetrate the aerogel and reach a plastic scintillator. A coincidence between the aerogel and the scintillator generates a gate signal for the ADC. The discriminators have an upper and lower threshold, allowing selection of specific energy intervals. The analog PM signal from the aerogel cell is amplified and fed into the Ortec ADC. In this arrangement it is unlikely for an electron itself to trigger. The electron energy spectrum is a typical $\boldsymbol{\beta}$ spectrum with an endpoint energy of $3.7 \mathrm{MeV}$. For $n=1.032$ aerogel, electrons above $2 \mathrm{MeV}$ produce Cerenkov photons.

A spectrum recorded with this arrangement is shown in Figure 46, indicating the observation of Cerenkov photons with a photomultiplier. Schindler has measured 5 (12) p.e. $/ \mathrm{cm}$ in $n=1.025(1.055)$ aerogel wit.s photomultiplier readout. ${ }^{44}$ Since the PMs were equipped with regular borosilicate windows, the sensitivity was restricted to photons above $350 \mathrm{~nm}$. In order to ineasure timing properties of different readout systems. the logic signal from the scintillator starts a TDC which is stopped by the logic signal from the aerogel. 
Figure 47. Test setup for reading out an NE 11] scintillator with a $Y 7$ fiber and a single avalanche photodiode.
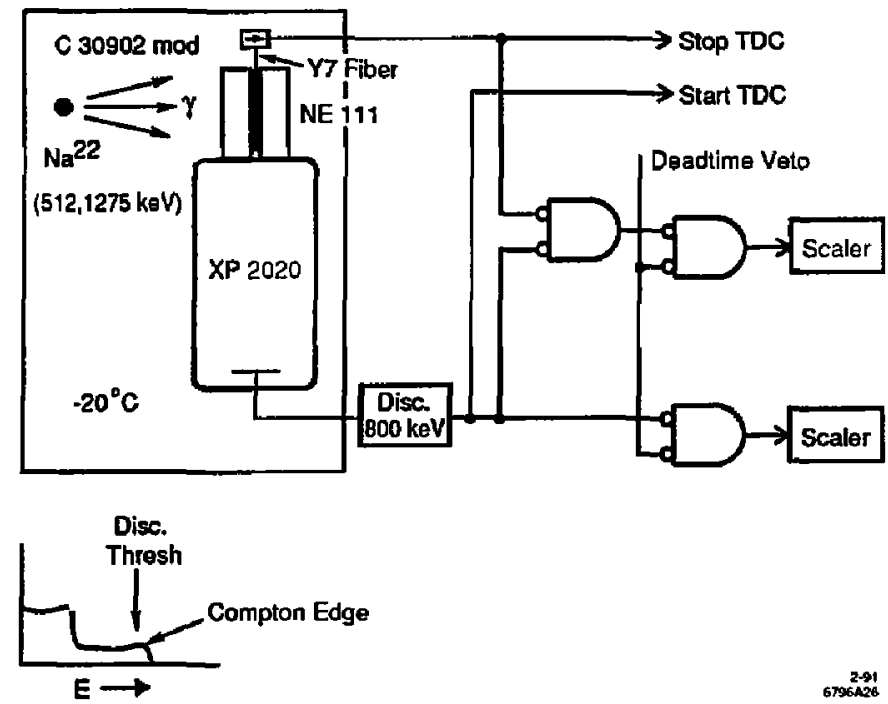

$67960^{292}$

Initial tests to determine the efficiency of the FFC/ SPAD readout were made by $E$. Lorenz at the MaxPlanck Institute in Munich. ${ }^{50}$ Figure 47 shows the experimental arrangement. A Y7 fiber from Kuraray was inserted into a block of plastic scintillator NE 111, which was read out with an XP 2020 PM on one end. On the other end the Y7 fiber was attached to an RCA SPAD (C 30902). The absorption spectrum of the $Y 7$ fiber in comparison to emission spectrum of the NE 111 scintillator is shown in Figure 48. The PM signal after passing through a discriminator with a threshold set at $800 \mathrm{keV}$ started a TDC. This value was chosen to trigger on the Compton edge of the $1275 \mathrm{keV}^{22} \mathrm{Na}$ line. Sufficient light. from this $\gamma$ line is produced in the scintillator to trigger the TDC. Some of these photons were absorbed in the fiber and re-emitted at an angle snaller than the critical angle and therefore travelled inside the fiber. The scin-

An efficiency of $99.08 \%$ has been measured for the FFC/SPAD readout combination at $-20^{\circ} \mathrm{C}$, after deadtime correction. tillator end of the fiber contained a mirror. All plotons thus ever' "rally reached the SPAD, unless they were lost by anoth - absorption and re-emission at an angle larger than tine critical angle. The SPAD output provided the stop signal for the TDC. In addition, the SPAD signals 
coincident with the PM signal and the PM signals themselves were counted both with and without a SPAD deadtime veto. The efficiency, the ratio of the two numbers, is $90 \%$ at room temperature. Including a dead-time veto for the SPAD, increased the efficiency to $98 \%$ at room temperature. Additional cooling to $-20^{\circ}$ improved the efficiency to $99.98 \%$. The decay time spectrum of the Y7 fiber is shown in Figure 49. The time resolution for the TDC measurements was $\sigma=1.3 \mathrm{~ns}$ at room temperature and $0.9 \mathrm{~ns}$ at $-20^{\circ} \mathrm{C}$. These results obtained in a high statistics environment, look very encouraging. However, a few deficiencjes, which will affect the efficiency of the readout of a thresbold Cerenkov counter, need to be remedied: a low dye concentration in the $\mathrm{Y} 7$ fiber, a spectral mismatch between the $Y T$ fiber and the SPAD (see Figures 48 and 56), a rather long decay time of 9.5 ns of the Y7 fiber, the coupling between the fiber and the SPAD, and area mismatcl of the fiber and the SPAD by a factor of 4 .

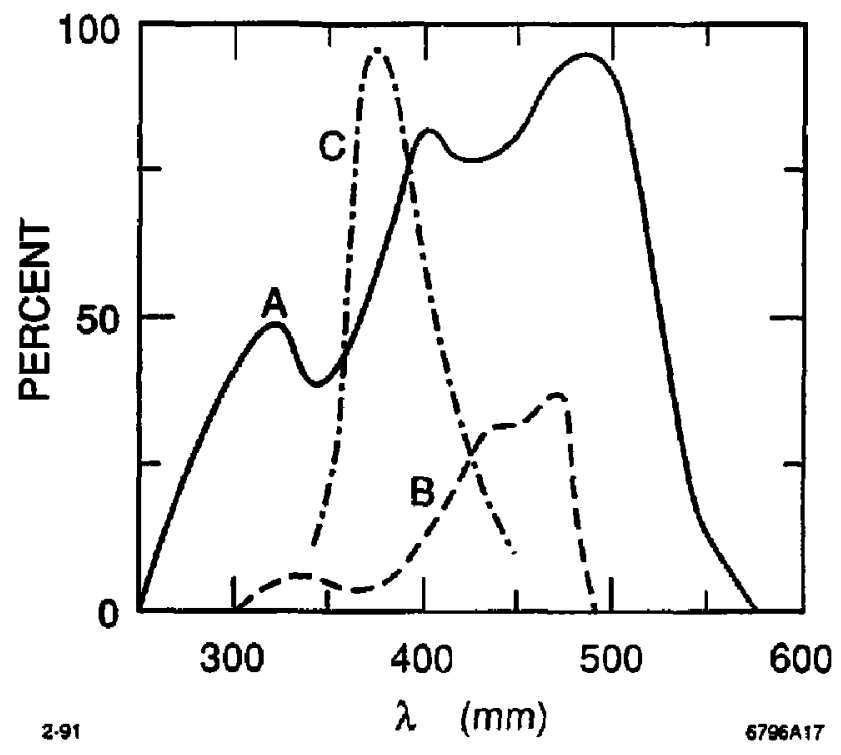

Figure 48, $A b$ sorption spectra of the $Y 7$ fiber and two laser dyes, BASF \#84 and \#241 in comparisoll to the NE 111 emission spectrum. 
Figure 49. Fluorescent decay-time spectrum of the YJ fiber.

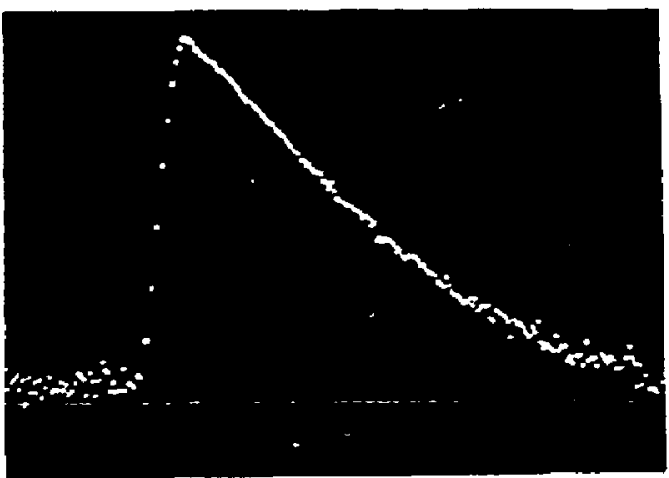

The Y7-fiber plus SPAD readout was then used in another test, to record Cerenkov photons in a $2.5 \times$ $2.5 \times 2.5 \mathrm{~cm}^{3}$ block of aerogel with a refractjve index of $n=1.038$. The setup is shown in Figure 50. The Cerenkov photons are produced by traversing electrons, which are emitted from a ${ }^{106} \mathrm{Ru}$ source. The aerogel was housed in an light-tight container with white walls coated with POPOP. A plastic scintillator, read out by an XP $2020 \mathrm{PM}$, was used as a reference. The discriminator threshold was set at $1.8 \pm 0.2 \mathrm{MeV}$, which is the threshold for electrons in aerogel with $n=1.038$. Cerenkov plotons were then counted by requiring a coincidence between the SPAD and the plastic scintillator. In this configuration, a coincidence rate of $3.8 \%$ was measured after correction for accidentals and direct hits. In order to build a successful device, the coincidence rate needs to be pushed to nearly $100 \%$. Since in this first test the cell design and readout were far from being optimal, there is much room for improvement, as can be seen in Tuble 10. The largest gains could be obtained by matching the areas of the fiber and SPAD, increasing the wall reflectivity from 0.85 to $>0.95$, increasing the sensitivity of wavelength detection and increasing the dye concentration in an unclad fiber. 


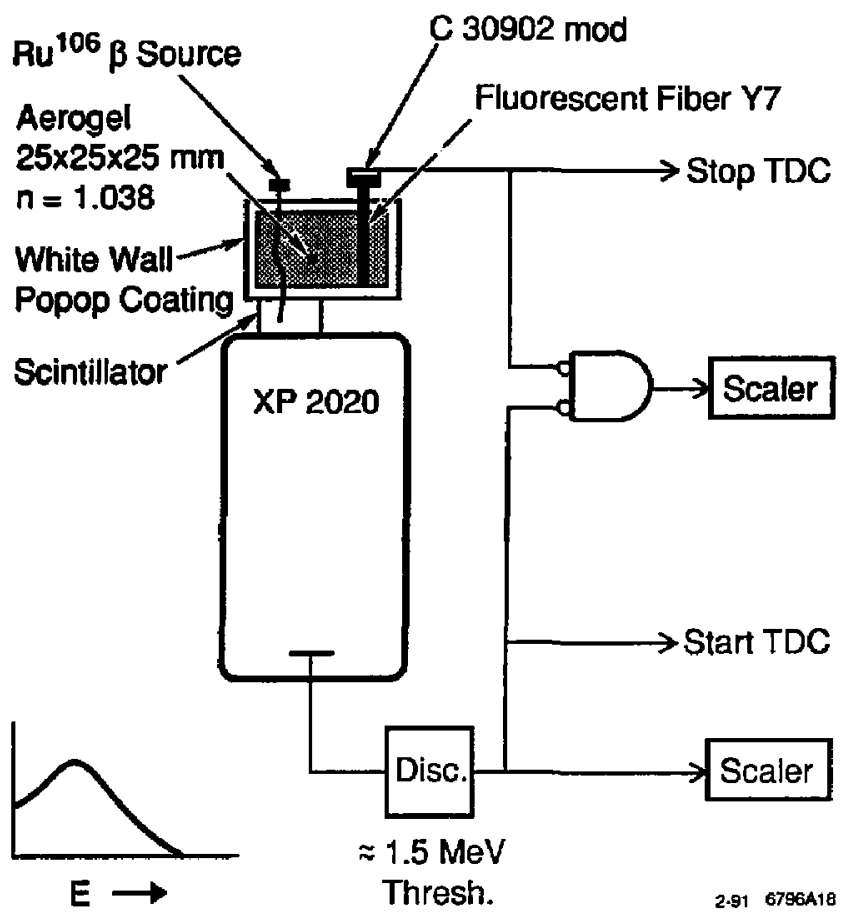

Figure 50. Test setup for measuring the effciency of an aerogel cell read out with a Y7 fiber and a SPAD.

In order to measure the wavelength dependence of the efficiency directly for an aerogel cell read out with a fluorescent fiber, E. Lorenz illuminated a $3 \times 3 \times 4 \mathrm{~cm}^{3}$ block of aerogel with $n=1.032$ in a spectrophotometer. ${ }^{50}$ The aerogel was housed in a light-tight container with about $90-95 \%$ of its walls covered with highly reflective Teflon. The light beam selected by a monochrometer from a Xe lamp was fed in through a $1 \mathrm{~cm}^{2}$ hole in the container. The aerogel was read out with a $1 \mathrm{~mm}$ thick unclad Y7 fiber containing additional BASF dyes \#84 and \#241, ${ }^{51}$ each with a concentration of $400 \mathrm{ppm}$. The fiber was read out by a $1 \mathrm{~cm}^{2}$ Hamamatsu photodiode. For normalization, the direct photon flux onto the photodiode without the cell was measured. Figure 51 shows the resulting efficiency distribution. In the visible region the efficiency plateaus at $2.3 \%$ and drops rapidly below $370 \mathrm{~nm}$ to $0.2 \%$ at $250 \mathrm{~nm}$. The main loss is due to light backscattered through the hole, since for lower wavelengths the first scattering occurs closer to

An efficiency of $\sim 2 \%$ has been measured for FFC/PD readout. 
Figure 51. EIficiency of an aerogel cell read out with a fuorescent fiber and a photodiode as a function of wavelength.

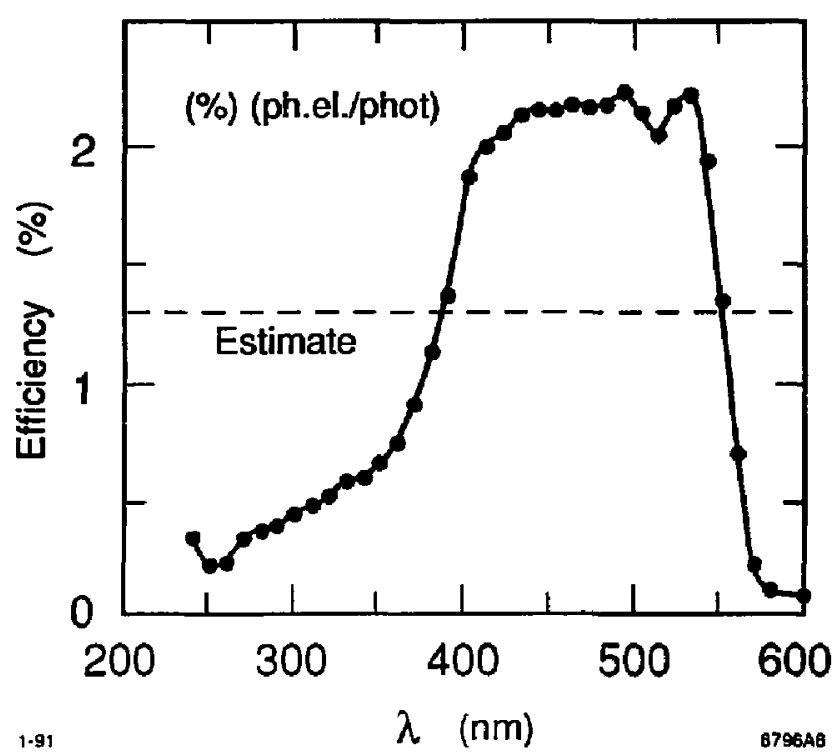

the hole. It has been shown that a reduction of the hole size leads to a considerable increase in efficiency at lower wavelengths. Other factors include a higher absorption probability in the aerogel, because both the path length and the absorption cross section become larger, and an increased absorption probability in the Teflon due to a smaller reflectivity. The cutoff at $550 \mathrm{~nm}$ is caused by the absorption cutoff of the dyes in the fiber as shown in Figure 48. The efficiency estimate for this setup was $1.3 \%$, which is consistent with the average of the measured distribution. In more recent measurements the efficiency was increased by another factor of 1.5, still leaving room for further improvements.

\subsection{Technical Issues}

Aerogel :Hixed with Wavelength Shifters

Mixing a wavelength shifter into the aerogel may yield a considerable efficiency increase, since both the scattering and absorption probabilities are reduced and the wavelengths are more closely matched to the absorp- 


\begin{tabular}{|l|c|c|c|}
\hline \multicolumn{1}{|c|}{ Item } & Present Condition & Possible Improvement & Gain \\
\hline All reflectivity & 0.85 & $>0.9 .5$ & 2 \\
Fiber properties & $1 \mathrm{~mm} \mathrm{clad}$ & $1 \mathrm{~mm}$ unclad & 1.2 \\
Fiber dye concentration & none & $>400 \mathrm{ppm}$ & $4-6$ \\
Dye self absorption & 0.4 & 0.5 & 0.8 \\
Absorption in fiber & $350<\lambda_{a}<480 \mathrm{~nm}$ & $240<\lambda_{a}<580 \mathrm{~nm}$ & 5 \\
Average emission & $500 \mathrm{~nm}$ & 600 & $1.3-1.7$ \\
Area fiber/SPAD & 0.25 & 1 & 4 \\
Distance fiber to SPAD & $1 \mathrm{~mm}$ & 0.3 & $\sim 2$ \\
Cooling & $-20^{\circ} \mathrm{C}$ & $-40^{\circ} \mathrm{C}$ & 1.3 \\
Optical coupling & $n=1.48$ & $n=1.58$ & 1.1 \\
Dye decay time & $9.5 \mathrm{~ns}$ & $<5 \mathrm{~ns}$ & 1.3 \\
\hline
\end{tabular}

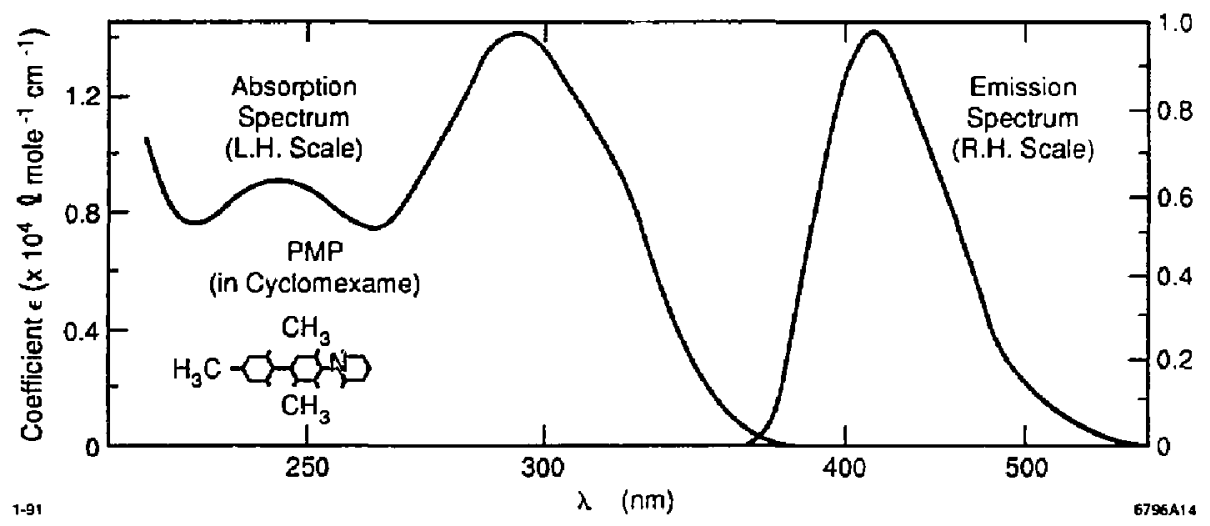

tion spectrum of the fiber. PMP seems to be a promising candidate for this purpose, since it has a rather large Stokes' shift, it dissolves in alcohol and the PXIP molecules $f_{i}$ well into the aerogel structure (sce below). POPOP is less suitable, because it has a lower Stokes shift. does not dissolves in alcohol. which is necessary for aerogel fabrication, and crystallizes in the aerogel. The efficiency is also much lower than for PMP. The absorption and emission spectra of PMIP are shown in Figure 52. The absorption range is from 220 to $370 \mathrm{~mm}$ with a peak at $290 \mathrm{~nm}$. The shifted light is in the $380-550 \mathrm{~nm}$ region with a peak a: $110 \mathrm{~mm}$. thus lying
Table 11. Improvements for the FFC plus SPAD rearlout.

Figure 52. Absorption and emission spectra of PMP.

PMP is a suitable wavelength shifter for mixing into awrougel. 
Figure 53, $A b-$ sorption and emission spectra of several PMP derivatjues.

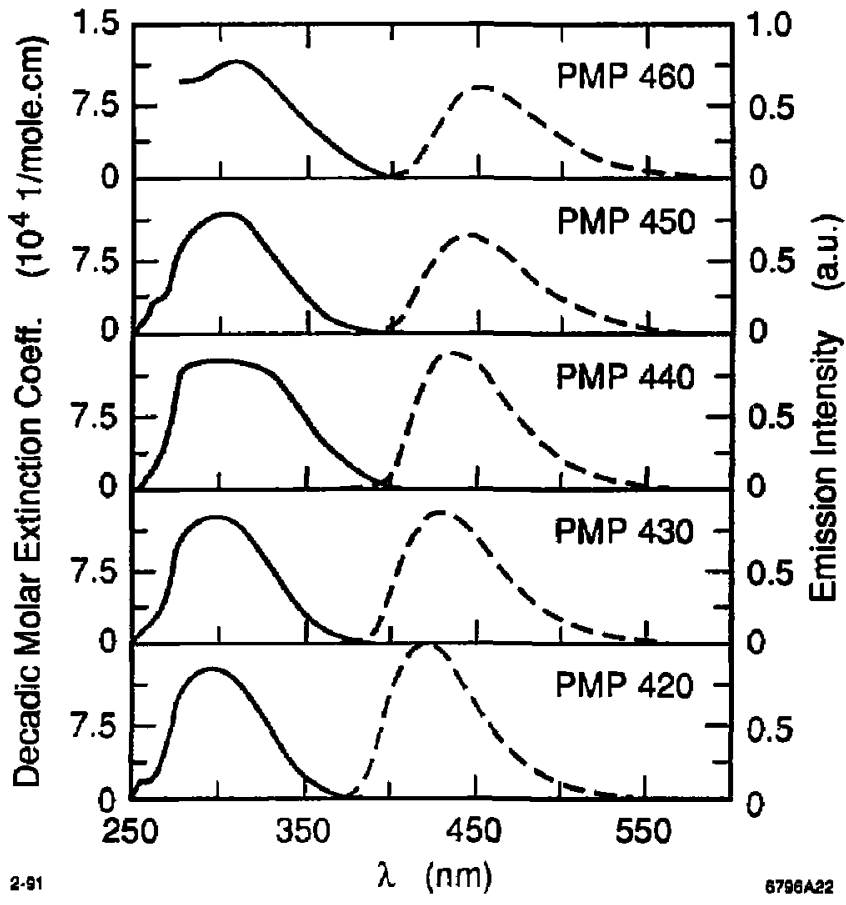

in the most sensitive range of photomultiplier cathodes (see Figure 61). The efficiency tor this process is high $(>80 \%)$. In the $220-330 \mathrm{~nm}$ range the extinction coefficient is greater than $7.5 \times 10^{3} 1 \mathrm{~mole}^{-1} \mathrm{~cm}^{-1}$. For a concentration of $300 \mathrm{ppm}$ of PMP and a silica aerogel of density $\rho=0.15 \mathrm{~g} / \mathrm{cm}^{3}$, this yields an attenuation length of $\Lambda<0.18 \mathrm{~cm}$. New derivatives of PMP have been developed recently; $;^{52}$ some of them may actually be even more suited for our purpose than PMP because of creu larger Stokes' shifts and higher efficiencies. Figure 53 shows the absorption and emission spectra of PMP derivatives, where PMP 460 is the WLS we are currently using. According to the figure, PMP 440 may yield a ligher efficiency, since the emission intensity is largest.

The production of aerogel with PMP is currently underway at Lawrence Livermore Laboratory. We have ob- 


\begin{tabular}{|c|c|c|c|c|c|c|c|c|c|}
\hline$\lambda[\mathrm{nm}]$ & (a) & (b) & (c) & (d) & (e) & (f) & (g) & (h) & (i) \\
\hline 250 & 95.0 & - & 37.0 & 72.5 & 89.0 & 4.9 & 91.5 & - & 71.0 \\
\hline 300 & 96.8 & - & 65.0 & 86.0 & 92.2 & 4.5 & 94.0 & - & 73.0 \\
\hline 350 & 97.8 & 95.5 & 80.5 & 92.6 & 94.5 & 6.0 & 95.8 & 95.0 & 83.0 \\
\hline 400 & 98.6 & 97.0 & 92.0 & 93.8 & 94.0 & 32.0 & 94.2 & 96.1 & 83.8 \\
\hline 450 & 99.0 & 97.8 & 94.1 & 94.0 & 93.2 & 86.8 & 92.9 & 96.3 & 82.5 \\
\hline 500 & 99.1 & 98.0 & 94.8 & 94.0 & 92.6 & 86.0 & 91.2 & 96.3 & 81.0 \\
\hline 550 & 99.2 & 98.0 & 95.0 & 94.2 & 92.5 & 85.0 & 90.0 & 96.0 & 80.0 \\
\hline 600 & 99.3 & 98.0 & 94.1 & 93.8 & 91.5 & 84.0 & 87.0 & 95.8 & 78.2 \\
\hline 650 & 99.3 & 97.9 & 94.0 & 93.8 & 91.0 & 84.5 & 83.5 & 95.6 & 77.0 \\
\hline 700 & 99.3 & 97.9 & 94.0 & 93.2 & 90.0 & 86.0 & 81.0 & 95.8 & 71.0 \\
\hline$\lambda[\mathrm{nm}]$ & (j) & (k) & (l) & (m) & (n) & (o) & $(p)$ & (q) & $(\mathbf{r})$ \\
\hline 250 & 71.0 & 6.3 & 40.8 & $\$ 1.0$ & 50.5 & 36.5 & 51.5 & - & - \\
\hline 300 & 73.0 & 6.5 & 43.5 & 85.5 & 80.0 & 67.0 & 81.0 & - & - \\
\hline 350 & 83.0 & 7.0 & 52.6 & 90.5 & 94.5 & 83.0 & 95.0 & 84.6 & S1.3 \\
\hline 400 & 83.8 & 42.0 & 58.2 & 91.2 & 95.5 & 92.5 & 95.5 & 82.5 & 85.2 \\
\hline 450 & 82.5 & 76.0 & 65.8 & 92.0 & 95.5 & 95.5 & 96.0 & 80.8 & 88.0 \\
\hline 500 & 81.0 & 77.0 & 72.8 & 92.0 & 95.4 & 96.0 & 95.5 & 79.1 & 89.8 \\
\hline 550 & 80.0 & 77.0 & 79.2 & 92.2 & 95.5 & 97.0 & 95.5 & 78.0 & 91.0 \\
\hline 600 & 78.2 & 76.8 & 83.0 & 91.9 & 95.0 & 96.2 & 94.8 & 77.0 & 91.4 \\
\hline 650 & 77.0 & 78.0 & 85.8 & 91.5 & 94.0 & 96.1 & 94.2 & 75.7 & 91.7 \\
\hline 700 & 71.0 & 77.0 & 87.0 & 91.0 & 93.8 & 96.0 & 93.8 & 75.2 & 91.8 \\
\hline
\end{tabular}

tained 4 pieces: two $0.5 \mathrm{~cm}$ thick discs, $4 \mathrm{~cm}$ in diameter, and two $3.4 \mathrm{~cm}$ long cylinders, $1.1 \mathrm{~cm}$ and $1.2 \mathrm{~cm}$ in diameter. Except for the smaller cylinder which has a density of $0.27 \mathrm{~g} / \mathrm{cm}^{3}$ all other pieces have $\rho=0.15 \mathrm{~g} / \mathrm{cm}^{3}$ The refractive indices for the pieces are $n=1.05 T$ and $n=1.032$, respectively. The PMP concentration of each piece is $300 \mathrm{ppm}$. Figure 54 shows the transmission measured with one of the discs. Comparing this to the transmission of pure aerogel shows that the aerogel doped with PMP is considerably more transparent. A $3 \times 3 \times 6 \mathrm{~cm}^{3}$ block of aerogel containing $300 \mathrm{ppm}$ of PMP will be made in the near future.
Table 12. Reflectivity of white diffuse reflectors. (a) Kodak paint; (b) 2 sheets of 6-SFC millipore paper 0.4 HAWP; (c) millipore paper $0.45 \mathrm{HAWP}$; (d) $\mathrm{BaSO}_{4}$ paint; (e) Bicron milljpore paper $6 \mathrm{VHP} 0.22$; (f) Tefion on linot used by $M A C R O ;(\mathrm{g})$ Teflon 11 mils; (h) one sheet of 6-SFC millipore paper 0.45 HAWP; (i) Floropore FGLP 0.2; (j) millipore 0.1 VCWP; (k) white paint; (l) millipore JVLP 0.1; (m) millipore $6 V W P$ 0.22; (n) mil- 
lipore VCWP $0.1 ;$ (o) 2 sheets millipore HAWP 0.45 ;

(p) millipore VCWP 0.1

(q) 6SFC Floropore; (r) 6SFC

Duropore.

Kodak white paint is the best diffuse reffector. but it has problems with out. gassing.

\subsection{Reffectivity of Diffuse Refectors}

It is important to have highly-reflecting container walls, since the average number of reflections is rather large, especially at low wavelengths, where the number of Cerenkov photons is enhanced ..s $1 / \lambda$. The reflectivity has to be greater than $98 \%$ in the visible region and greater than $90-95 \%$ in the UV region to keep losses minimal. Table 12 lists the reflectivity of several materials in the $250-700 \mathrm{~nm}$ range. Figure 55 plots the wavelengtl dependence of the reflectivity for some white reflectors listed in Table 12. ${ }^{44}$ Most of the samples do not meet our requirements, because the reflectıvity is poor in the UV-light region or $\mathrm{R}<95 \%$ in the visible-light region. The liodak paint seems to be the best candidate, but outgassing of the organic solvent may cause problems. We have obtained a sample of the Kodak paint to perform extensive tests. For comparison, studies with white paints from Bicron and Nuclear Enterprise will be madc. Another promising candidate is two layers of 6-SFC millipore papet. This paper will be tested to ascertain whether the reflectivity remains above $90 \%$ in the $200-350 \mathrm{~nm}$ range. The two teflon samples shown in Figure 55 do not meet our requirements, though Teflon would be ideal, since outgassing and absorption of humidity are not an issue. It may therefore be interesting to investigate other tellon films, since it is likely that some have better reflective properties. Furthermore, if the losses are due to transmission rather than to absorption, the use of aluminized mylar behind the white reflector may increase the reflectivity. There are, however, some caveats, which may, in the end, disqualify teflon: if good reflectivity is only obtained by increasing the thickness, background levels from Cerenkov photons produced in the teflon layer may become intolerable ( $\sim 6$ photons are produced in the $220-550 \mathrm{~nm}$ range per $100 \mu \mathrm{m}$ thichiness). 


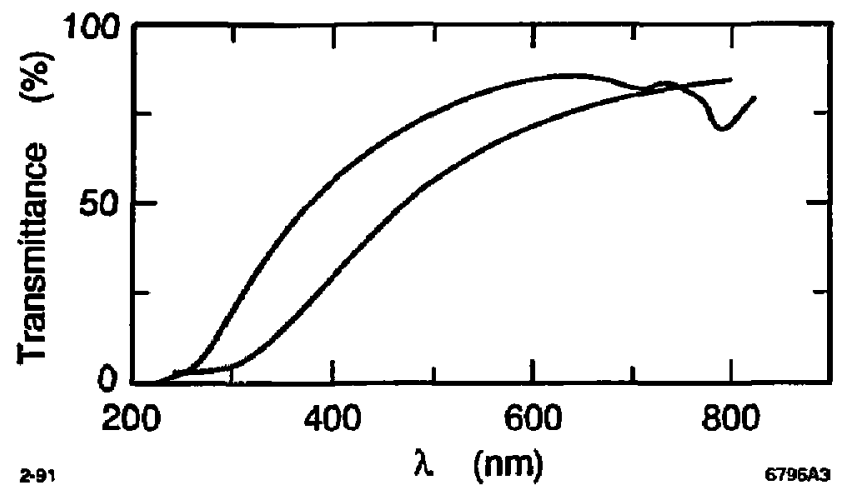

Figure 54. Transmission spectrum of aerogel mixed with PMP in comparison to pure aerogel.

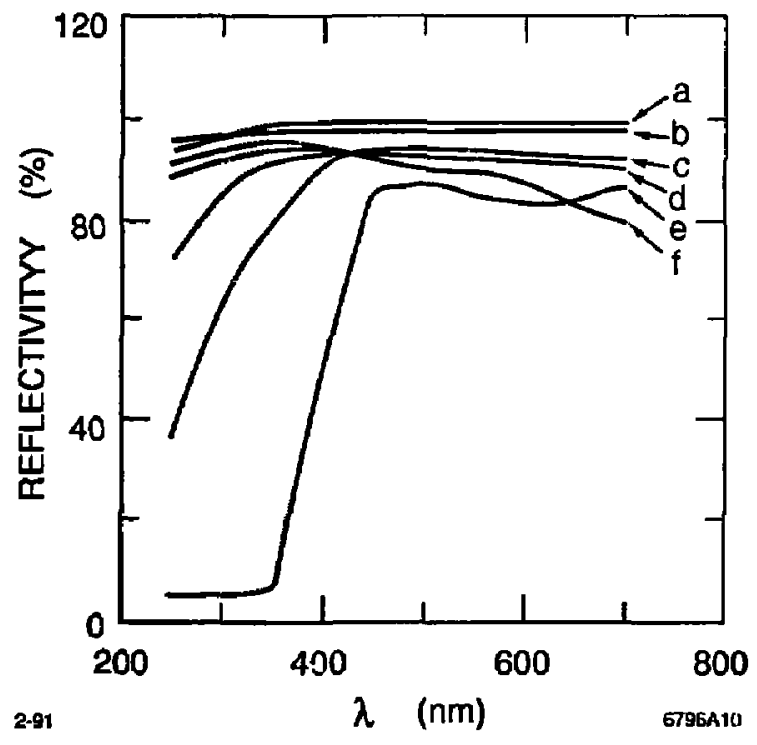

Figure 55. Reflectivity measurements of white difuse reflectors as a function of wavelength.

\section{Fluorescent Fibers}

In order to keep the efficiency high, the properties of the fluorescent fiber need optimization. The number of Cerenkov photons increases with $1 / \lambda$, while the sensitivity of the SPAD peaks at $800 \mathrm{~nm}$, as shown in Figure 56. Thus, the photon wavelengths have to be wavelength shifters for the FFC's. 


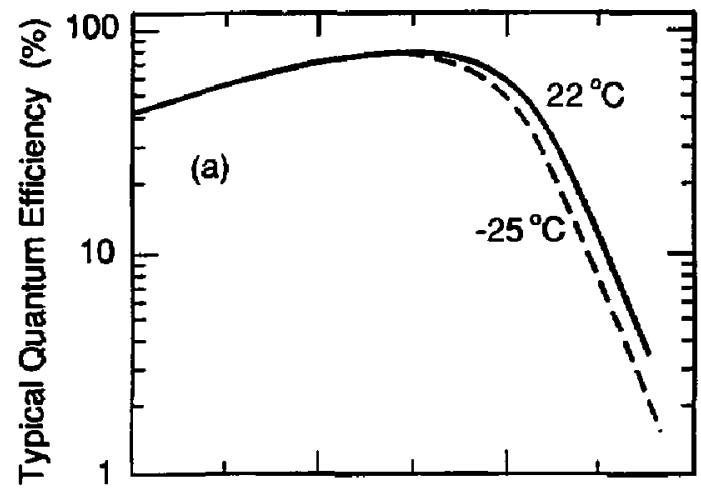

Figure 56. Spectral sensitivities of single photon avalanche diodes.
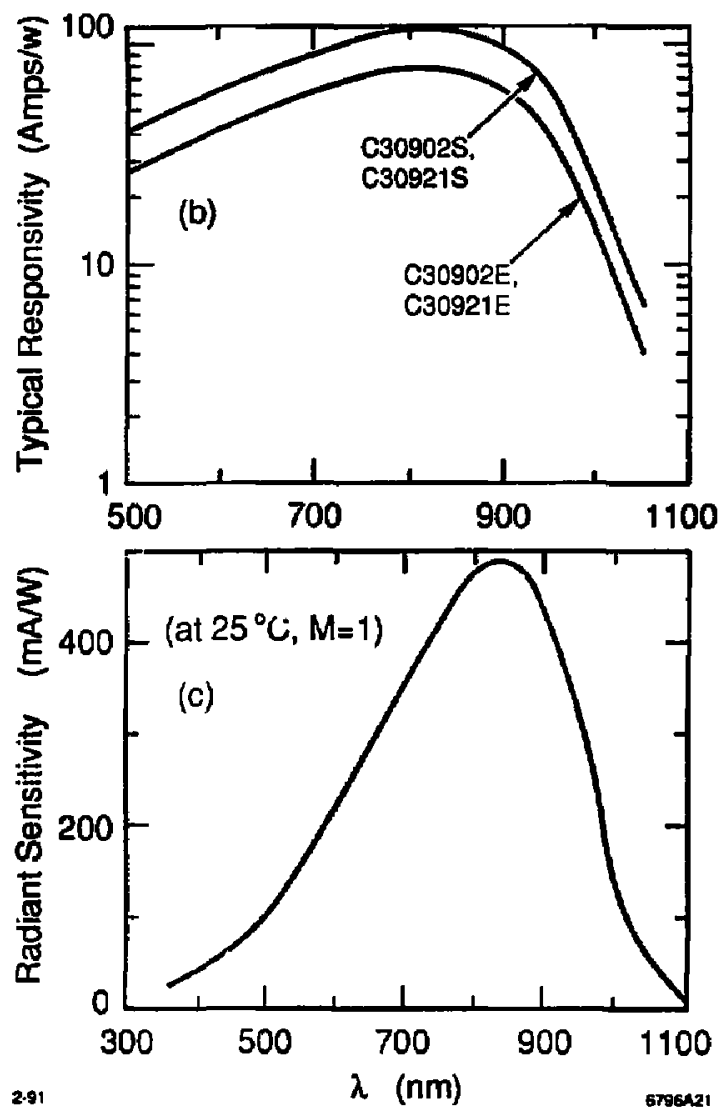
shifted by several hundred nanometers, which requires several shifts. The first shift to $450 \mathrm{~nm}$ is accomplished by PMP mixed in the aerogel. The remaining shifts have to done with several laser dyes, since their typical Stokes' shifts are only $30-50 \mathrm{~nm}$. The quantum yields of fluorescence, however, are typically greater than $95 \%$. In Figure 17 the absorption spectra of the BASF dyes \#84 and \#241 are shown in comparison of the absorption spectrum of the $Y 7$ fiber. From the figure it is evident that the dye in the Y7 fiber can be omitted if the dyes \#84 and \#241 are used. But it is important to add one or two more laser dyes, to increase the absorption range above $500 \mathrm{~nm}$. Appropriate dyes are made by BASF. ${ }^{51}$ These dyes are mixed into an unclad fiber made of the highly transparent and photochemically stable plastic Polymethylmethacrylate (PMMA). For cell sizes of 2$3 \mathrm{~cm}$, the optimal fiber diameter is about $1 \mathrm{~mm}$, since this leaves the fiducial volume large enough while keeping a moderate average path length. Dye concentrations of 300-400 ppm are probably sufficient for a $1 \mathrm{~mm}$ fiber diameter, as the absorption length is estimated to be of the order of a few tenths of a millimeter. A thinner fiber would require higher dye concentrations, which would increase the probability for other absorptions. Though a minimum number of absorptions is necessary to achieve the full shifting, too many absorptions result in an efficiency decrease, since photons are either lost by selfabsorption or a new emission angle allows them to leave the fiber. Thus, the transport efficiency inay be different in the two cells, since the fiber lengths differ by a ratio of 5 to 2. The fiber end located in the aerogel will have an end reflector made of aluminized mylar.

\section{Single Photon Avalanche Diodes}

We are curently investigating two avalanche photodiodes: the C30902S from RCA and the $\$ 2383$ from Hamamatsu. ${ }^{53}$ Their spectral response is shown in Figure 56. In order to obtain the best coupling of the FFC to the SPAD, the FFC-SPAD gap must be very sinall ( $<1 \mathrm{~mm}$ ). RCA SPADs have a borosilicate window which must be removed and replaced by a fiber-holder,
The SPAD's must be modified to optimize light collection. 


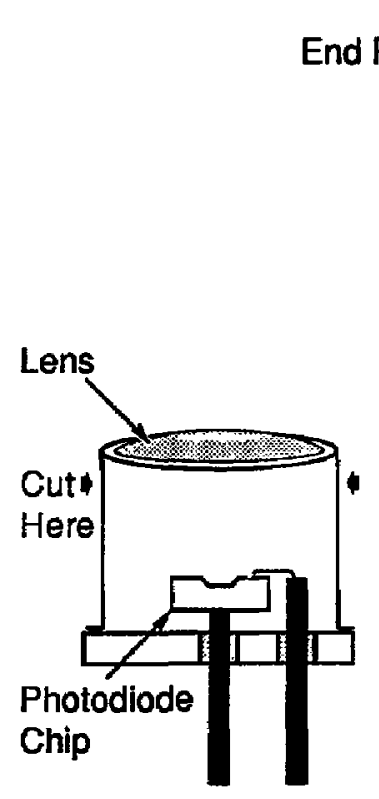

Original Photodiode
Figure 57. Mounting of the fiber to the avalanche diode.

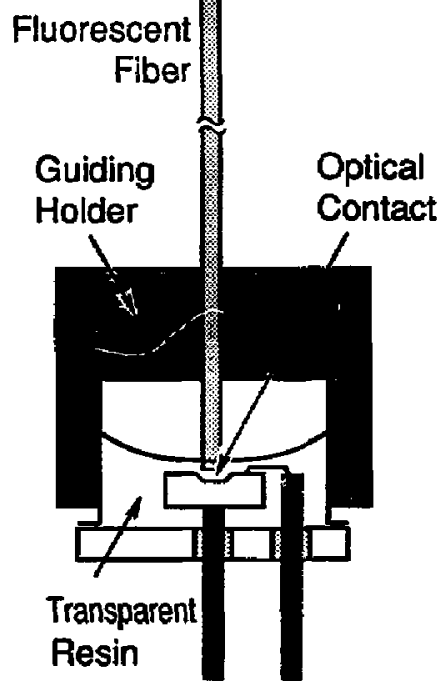

Modified Photodiode

B7\$616

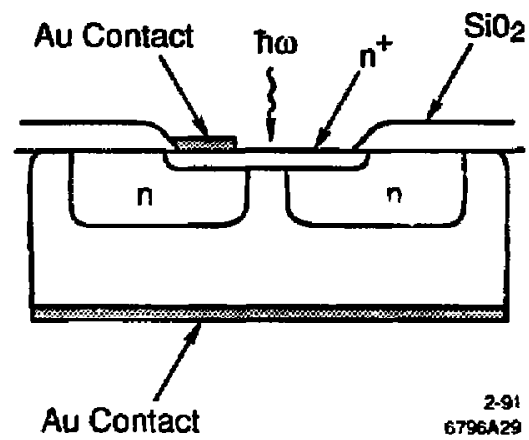

as sketched in Figure 57. To ensure a minimal gap without damaging the surface, a stop, which rests on the non-sensitive $\mathrm{SiO}_{2}$ surface, defines a constant gap. A cross-section of the RCA SPAD is shown in Figure 58. 
A major current deficiency is the mismatch of the diameters of the SPAD and the fiber. Apparently RCA is having problems with fabricating larger area SPADs. Since these prublems may be intrinsic to the design, it may be impossible for them to fabricate SPAD's with a $1 \mathrm{~mm}$ diameter. For the Hamamatsu avalanche photodjodes, operation in the Geiger mr le, which is crucial for obtaining a high gain of $>1 \mathrm{l}$.emains to be demonstrated. 'f, however, a $1 \mathrm{~mm}$ SPAD operating in the Geiger mode is not commercially available, one has two options: one can either develop a focussing system or use four $0.5 \mathrm{~mm}$ thick fibers. For both cases, efficiency losses are inevitable; the second option also increases costs.

The SPADs work best if they are cooled to $-20^{\circ} \mathrm{C}$ to $-40^{\circ} \mathrm{C}$. The noise rate drops from $\sim 12 \mathrm{kHz}$ at room temperature to $\sim 500 \mathrm{~Hz}$ at $\mathrm{T}=-20^{\circ} \mathrm{C}$, ds shown in Figure 59(a). Thus, for optimal performance, a cooling system is required. It is certainly not as involved as a cryogenic system, but proper insulation is needed, since moisture will condense near the cooling liwes. Though the noise level of the RCA SPADs was tolerable at room temperature, one may in fact use cheaper SPADs in the real detector, which may be too noisy at $20^{\circ} \mathrm{C}$. The coincidence technique does not require a cooling system. It will be necessary to demonstrate that cross-talk between the fibers is negligible. It may, in any case, be necessary to lnep the humidity at a low level in order to avoid damage to the aerogel. This is achieved with a steady flow of nitrogen, which may be cooled to $-20^{\circ} \mathrm{C}$.

Due to the long deadtimes (> $300 \mathrm{~ns}$ ) required to quench an avalanche, the RCA SPAD's can detect only a single photon. If enough Cerenkov photons are present and noise levels are low, the detection of one photoelectron is sufficient for particle identification. However, it would be helpful to have some redundancy by observing more than one pliotoelectron. This would be possible if the dead time is reduced to a few hundred picoseconds. In the RCA SPADs the long dead time is needed to reduce the probability for afterpulsing. Figure 59 (b) shows the probability for afterpulsing in the 100-300 ns range. The curve indicates that a minimum dead time must be
A mismatch between the diameters of the fiber and the SPAD causes loss of efficiency.

Cooling of RCA SPAD's considerably reduces noise.

Improvements by Hamamatsu may allow counting of all Ćerenkov photons. 
Figure 59.

(a) Dark current as a function of temperature; (b) probability of after pulsing as a function of dead time.
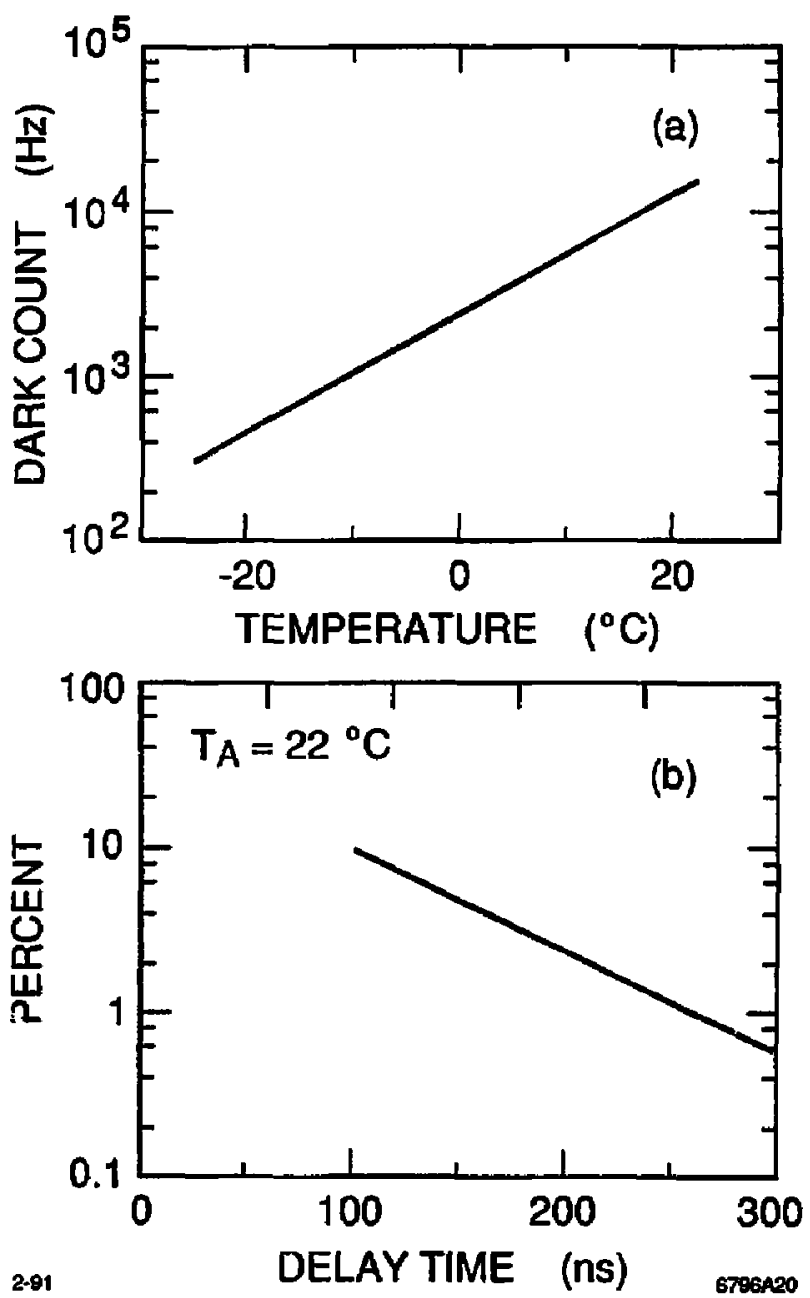

300 ns. Hamamatsu, however, claims that it may be possible to achieve dead times less than $1 \mathrm{~ns}^{54}$ Hamamatsu further claims that their avalanche photodiodes show a lower after-pulsing rate at room temperature than when cooled, while noise levels at room temperature are lower than for the RCA SPAD's. Thus the Hamamatsu PDs may be the ideal photon detectors; it is expected that 
they will soon become available.

\section{Phototetrodes}

A phototetrode (PT) is a 4 stage photomultiplier, which is can operate in magnetic fields up to $1 \mathrm{~T}$. The gain at nominal operation of $1 \mathrm{kV}$ is 50 at zero field; it is 12.5 at $1 \mathrm{~T}$. The gain is largest if the PT is oriented nearly parallel to the $\overrightarrow{\mathbf{B}}$ field, while it drops to zero if the PT is perpendicular to $\overrightarrow{\mathrm{B}}$. The gain as a function of the direction of the PT is shown in Figure 60. For a $30^{\circ}$ angle, the gain is $75 \%$ of the maximum gain. Since the gain variations are still relatively small at $30^{\circ}$, this seems to be a suitable operation point. Operation at $45^{\circ}$ is desirable, even though the gain is still 0.5 , as the variation of gain with angle is very large. These PTs are obtainable with a multialkali photocathode. Figure 61 shows the quantum efficiency of a multialkali cathode for a borosilicate and a quartz window in comparison to the PMP emission spectrum. If PMP is mixed into the aerogel, a multialkali cathode is important but a quartz window is not essential.

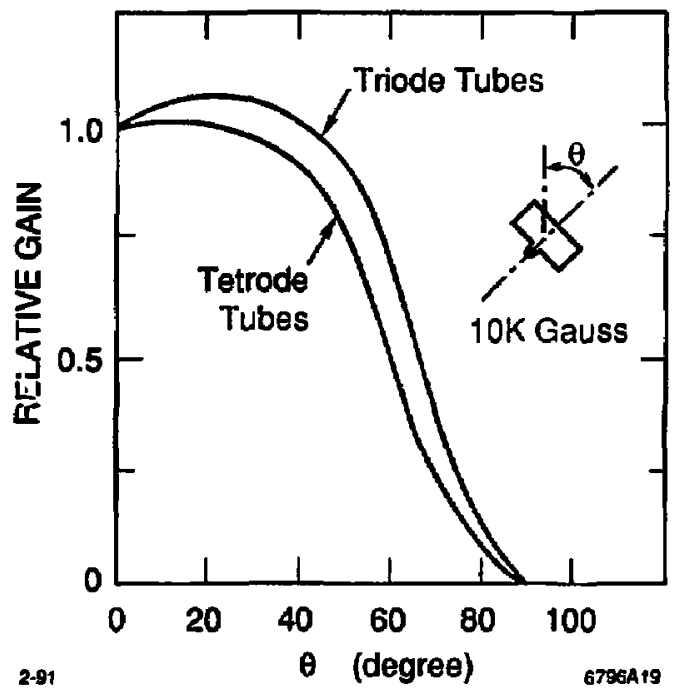

Phototetrodes must be operated at angles of les than $45^{\circ}$ to the magnetic $f$ I.

Figure 60. Relative gain of a phototetrode versus its angle with the magnetic field. 
Figure 61. Quantum efficiency of a multialkaline cathode compared to the PMP emission spectrum.

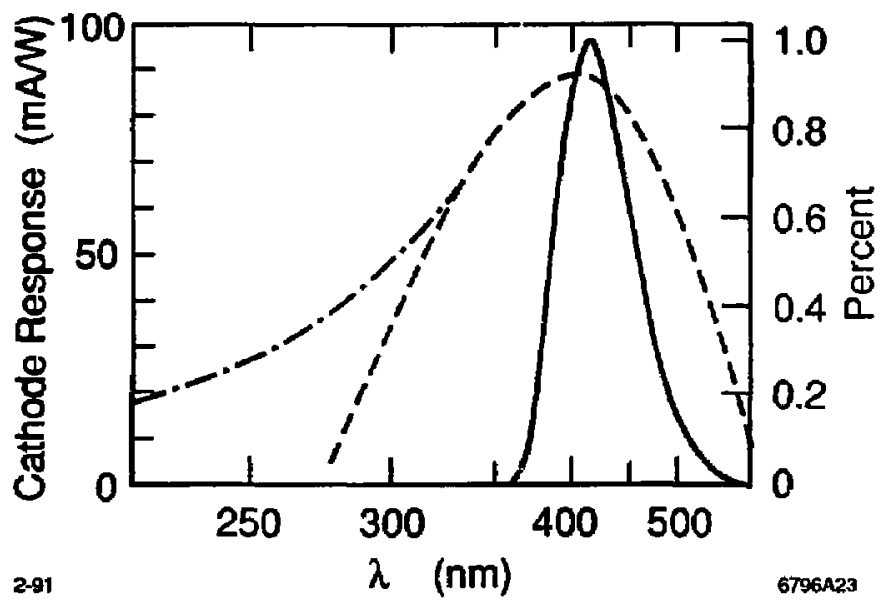

\begin{tabular}{|l|c|c|c|c|}
\hline \multicolumn{1}{|c|}{ Material } & Thickness $[\mathrm{cm}]$ & Density $\left[\mathrm{g} / \mathrm{cm}^{3}\right]$ & $X_{0}\left[\mathrm{~g} / \mathrm{cm}^{2}\right]$ & Rad. Length [\%] \\
\hline Aerogel 1.008 & 10 & 0.038 & 29.85 & 1.27 \\
Aeroge] 1.06 & 4 & 0.286 & 29.85 & 3.83 \\
Mylar & $4 \times 0.005$ & 1.39 & 39.95 & 0.07 \\
Cardboard & $4 \times 0.015$ & 0.6 & 40.0 & 0.09 \\
White paint & $4 \times 0.0025$ & 4.5 & 9.91 & 0.45 \\
Mech. support & $2 \times 0.1$ & 1.2 & 40. & 0.6 \\
\hline
\end{tabular}

Table 13. Inactive material of a two-cell aerogel threshold Cerenkov counter.

The inactive material for a two-cell aerogel threshold Cerenkov detector amounts to $6 \%$ of a radiation length.

\section{An Estimate of Inactive Material}

In order to estimate the inactive material in front of the electromagnetic calorimeter resulting from an aerogel Cerenkov counter, we have used the cell design described in Section 3. Table 13 gives a detailed list of the individual components. We have assumed that the mechanical support structure consists of two layers of carbon fiber.

Thus, the total inactive material amounts to about $6 \%$ of a radiation length. The estimate for white paint is based on $\mathrm{BaSO}_{4}$. Using Teflon or millipore paper as a reflector reduces the inactive material by about $0.5 \%$. The overall contribution from the fiber and SPAD is negligible. For the tetrode readout the inactive material increases by $1-2 \%$, depending on the mechanical support structure. For both designs, this is considerably less inactive material than needed for a CRID/RICH particle identification system. 


\subsection{Performance of Threshold C̈erenkov Counters}

In concluding this section, we will demonstrate that a two-cell threshold Cerenkov counter with refractive indices of $n=1.06$ and $n=1.008$ provide sufficient particle identification to achieve the physics aims of an asymmetric $\mathrm{B}$ Factory. The probability for observing a single photoelectron from a sample of Cerenkov photons produced by a charged particle of mass $m$ traversing an aerogel cell with a momentum $p$ above threshold $\left(\beta>\beta_{t}=1 / n\right)$ is given by:

$$
P_{e}=1-\exp \left(-\bar{N}_{e}\right)
$$

where $\bar{N}_{e}$ is the average number of photoelectrons, which is determined from the maximal number of photoelectrons, $N_{\text {max }}$, expected at $\beta=1$, by:

$$
\bar{N}_{e}=N_{\max }\left(1-\frac{\beta_{t} \gamma_{l}}{\beta_{\gamma}}\right)
$$

For the two cells, $N_{\max }$ is assumed to be 10 and 5 photoelectrons, respectively.

Figure 62 plots the probability ${ }^{55}$ for detecting electrons, muons, pions, kaons and protons as a function of momentum. Solid curves correspond to the $n=1.06$ cell while dashed curves correspond to the $n=1.008$ cell. False identifications due to noise and $\delta$-rays produced by particles below threshold are neglected in this estimate. From our previous discussion we know that noise is small. The production of $\delta$-rays is expected to be also small, but needs further study. If one requires an identification probability of $>50 \%, K / \pi$ separation is provided in the 0.4-4.2 GeV/c momentum range;

For $\pi / e$ and $\pi / \mu$ separation as well as $K / p$ separation in the 1-1.4 GeV/c region and above $2.7 \mathrm{GeV} / \mathrm{c}$, one needs additional information: lower-momentum electrons are identified by $d E / d x$, while higher-momentum electrons can be identified by pattern recognition in the electromagnetic calorimeter; for muon identification a hadronic calorimeter or a muon range out system has to be used; antiprotons leave a clear signature in the
For an identification probability above 50\%, aerogel cells with indices of $\mathrm{n}=$ 1.06 and 1.008 provide $K / \pi$ separation between 0.4 and $4.2 \mathrm{GeV} / \mathrm{c}$. 
Figure 62, Prob. abjlity of detecting particles in a two-cell threshold Cerenkov counter detector.

A two-cell aerogel Cerenkor detector, supplemented by $d E / d x$ measurement in the drift chamber, provides excellent particle identification for an asymmetric B Factory.

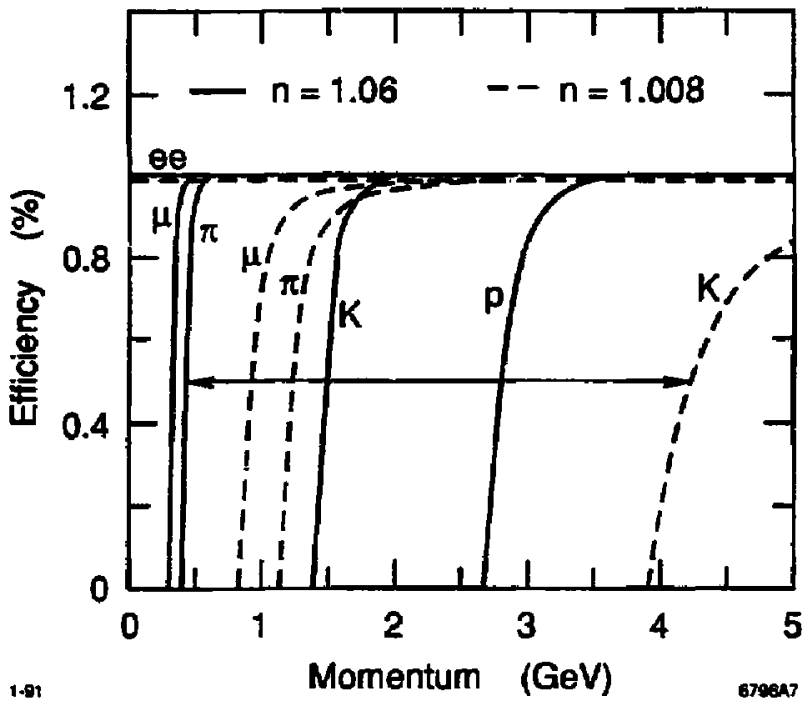

electromagnetic calorimeter. A residual problem for detecting protons above $2.7 \mathrm{GeV} / \mathrm{c}$ remains unsolved.

The performance of a two-cell aerogel threshold Ceren. kov counter has also been calculated for typical $B \bar{B}$ events. An example is shown in Figure $63 .^{56}$ Both the $B$ and $\vec{B}$ decay semileptonically. The final state consists of $\nu_{\mu} \mu^{+} \pi^{-} K^{+} \pi^{-}, \bar{\nu}_{e} e^{-} \pi^{+} K^{-} \pi^{+}$.

Figure 64 shows the performance of a $d E / d x$ system, a TOF system, a CRID system and two-cell threshold Cerenkov counters to identify all charged particles in the above event. The $d E / d x$ simulation was based on 32 one $\mathrm{cm}$ samples taken in the drift chamber operated with a $\mathrm{He} /$ isobutane/ $/ \mathrm{CO}_{2}$ gas at atmospheric pressure. The resolution was assumed to be $7.5 \%$ and a plateau to minimum ratio of 1.4. For the TOF system, a barrel and two endcaps of $5 \mathrm{~cm}$ thick scintillation counters have been assumed, with a resolution of $75 \mathrm{ps}$ and an efficiency of $100 \%$. The CRID simulation is based on $1 \mathrm{~cm} C_{6} F_{12}$ liquid radiator $(n=1.277)$ assuming 36.6 observed photons out of 61 produced. The CRID angle is assumed to be measured with $100 \%$ efficiency. The simulation of a two-cell threshold Cerenkov counter system is based on 


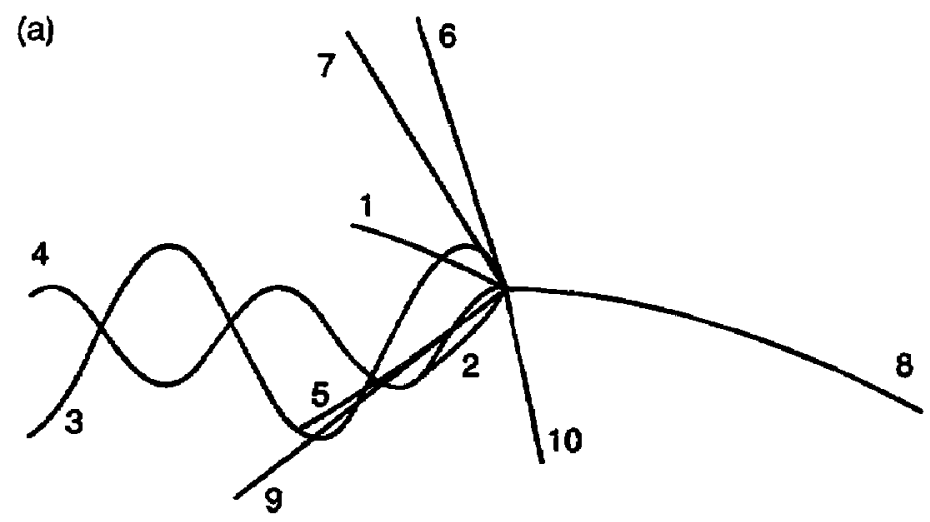

(b) $T(4 S) \rightarrow \bar{B} B$

(c)

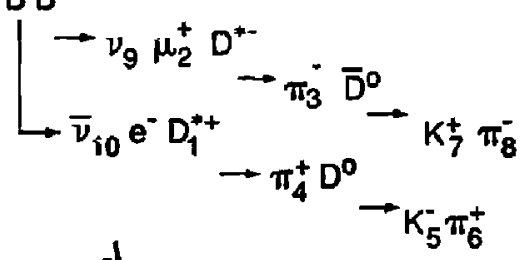

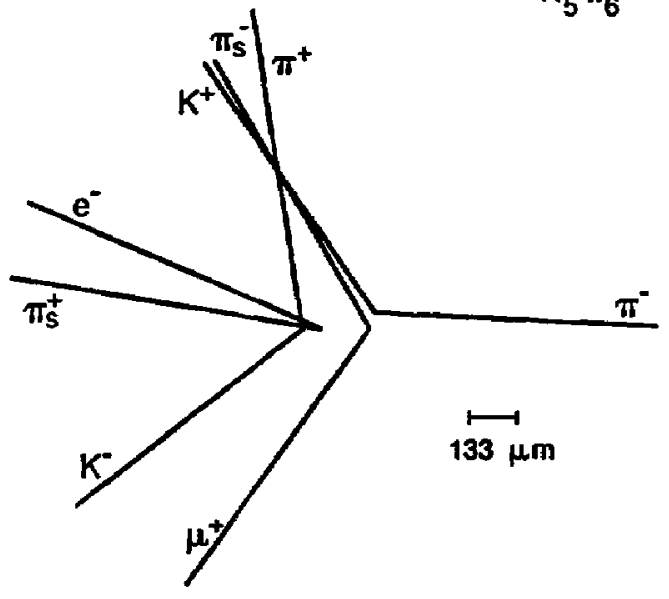

the parameters given above.

Particles 1, 3, 4 are well identified by $d E / d x$. Both the CRID and the two-cell threshold Cerenkov counter do a good job in identifying the remaining particles, while TOF identification is limited to momenta below 
1.5 GeV/c and thus cannot classify all of the remaining particles. A $d E / d x$ system and a two-cell threshold Cerenkov counter provide good $K / \pi$ separation up to $4.2 \mathrm{GeV}$.

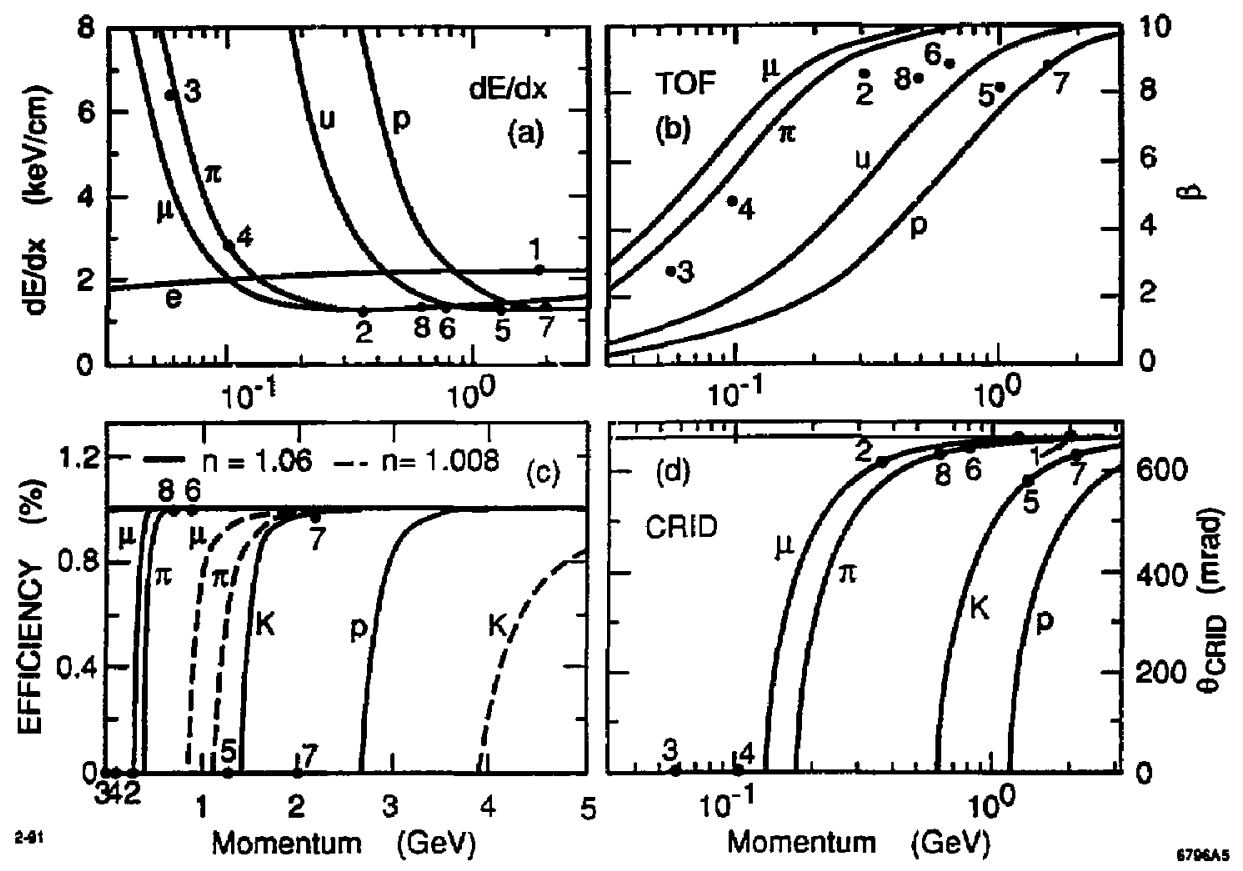

Figure 64. Performance of (a) $d E / d x$, (b) TOF, (c) CRID/RICH, and (d) a two-cell threshold Cerenkov counter detector for identifying the particles of the decay in Figure 63.

\subsection{Conclusions and Outlook}

In conclusion, threshold Cerenkov counters using two cells of aerogel with refractive indices of $n=1.06$ and $n=1.008$ provide a potential alternative to CRID/RICH counters. The inactive material for the entire device is only a few percent of a radiation length, thus leaving the good energy resolution in the electromagnetic calorimeter unaffected. Though the initial test results look very encouraging, a lot of work remains to be done before this idea can be turned into a large scale dete tor system. The most important issues are whether one actually 
can detect enough photons, whether the readout is suffciently reliable and efficient to build a particle iclentification system and whether aging effects due to radiation, humidity and absorption of organic molecules are serious. Our main goal for the near future is to investigate each of these issues. Specific R\&D items include:

- Both the absorption and scattering length have to be measured in different samples, examining the dependence on $\rho$, monitoring time dependence and studying the transmittance after exposure to various paints, wavelength shifters and high doses of radiation. It is important to obtain more understanding of which precautions are necessary for the construction of a large detector.

- The properties of aerogel mixed with PMP have to be studjed focussing on questions like: does PMP scintillate? What is the optimal PMP concentration? Does radiation change the PMP bounds and therefore damage the aerogel? Are there other long-term aging effects? Is the PMP concentration uniformly distributed? In order to find the best radiator, aerogel containing other PMP derivates and the wavelength shifter $\mathrm{HF3}$ will also be made and tested.

- In order to optimize the efficiency, it is necessary to conduct various material studies: (i) measure the flux concentration in the fiber by testing different laser dyes; (ii) optimize the coupling of the fiber and the SPAD; (iii) measure the reflectivity of white diffuse reflectors; (iv) find the optimal container; (v) test different SPADs and PT.

- Both the photoelectron yield and efficiency have to be measured in several $n=1.032$ aerogel cells with and without PMP. For readout a UV sensitive PM, a PT and a FFC plus SPAD will be used, for a direct comparison.

- Once the best readout is found, a test array for $n=1.008$ aerogel will be built and tested. If this test is successful, we will build a two-cell prototype
Aerogel threshold counters may provide an interesting alternative to ring-imaging devices. 
array consisting of $n=1.008$ and $n=1.06$ cells and do a beam test using pions and kaons.

We have established a collaboration with L. Hrubesh of Lawrence Livermole Laboratory, which will emphasize the production and testing of aerogel incorporating various wavelength shifters.

\section{ACKNOWLEDGEMENTS}

L. Hrubesh provided us with numerous samples of aerogel. We would like to thank L. Hrubesh, E. Lorenz, S. Schindler and A. Vorobiov for fruitful discussions. We also wish to thank $A$. Weinstein for generating several plots and J. Chiu for helping with some of the aerogel measurements. 


\section{REFERENCES}

1. For a discussion of these final states, see SLAC353, "The Physics Program of a High Luminosity Asymmetric B Factory at SLAC," p. 133-141, and F. Le Diberder, Ba $\vec{B} a r$ Note \# 33, "Background Study of the $B \rightarrow \pi^{+} \pi^{-}$Channel."

2. R. Aleksan, et al., Phys. Rev. D39, 1283 (1989).

3. See, for example, W. B. Atwood, in Proceedings of the Summer Institute on Particle Physics, (1980) SLAC-Report-239.

4. R. Stroynowski, in Proceedings of the Tau-Charm Factory Workshop, May 23-27, 1989, SLAC, Stanford, CA, SLAC-343 (1989).

5. See, for example, S. E. Willis, in Proceedings of the Symposium on Particle Identification at High Luminosity Colliders, (1989) Fermilab, Batavia, IL, April 5-7, 1989.

6. See, for example, S. Banerjee, et al., Nucl. Instr. and Meth. A269, 121 (1988).

7. Properties of most of these devices are discussed in "Major Detectors in High Energy Physics," Particle Data Group, LBL-91 Supplement UC34D (1985).

8. I. Lehraus, Nucl. Instr, and Meth. 217, 43 (1983).

9. W. Alison and P. Wright, Exp. Tech., in High

Energy Physics, Addison-Wesley Publ., 1987;

G. Lynch, LBL-TPC-81.

10. J. Va'vra, et al., Nucl. Instr. and Meth. 203, 109 (1982); J. Va'vra, Contribution to Proceedings of the Tau-Charm Factory Workshop, May 23-27, 1989 , SLAC-343.

11. The dependence obtained empirically by fitting argon data presented in I. Lehraus, R. Matthewson, and W. Tejessy, Phys. Scr. 23, 727 (1981).

12. See, for example, J. V. Jelly, "Cerenkov Radiaiion and its Applications," (1958) Permagon Press.

13. J. Seguinot and T. Ypsilantis, Ring Imaging Cerenkov Detectors (1990), manuscript in preparation. 
14. J. Seguinot and T. Ypsilantis, Nucl. Instr. Meth. 142, 377 (1977).

15. SLD Collaboration, SLD Design Report, SLACREP-273 (1984).

16. T. Ypsilantis, Working Group on Particle ID at the Workshop on Physics and Detector issues for a High Luminosity Asymmetric B Factory, June 7, 1990, SLAC; see also T. Ypsilantis, $B a \bar{B} a r$ Note \#44.

17. J. Sequinot, et al., CERN EP-90-88, June 1990.

18. CERN/LEPC/\$3-3 (1983), CERN/LEPC/P2 (1983).

19. R. Arnold, et al., Nucl. Instr. Meth. A273, 466 (1988).

20. V. Ashford, et al., Proceedings of the XXIII International Conference on High Energy Physics, 1470 (1986).

21. R. Arnold, et al., Nucl. Instr. Meth. A270, 255 (19S8).

22. CERN/LEPC/84-15 (1984).

23. T. Ypsilantis, Phys. Scr., 23, 371 (1981).

24. B. Ratcliff, in Ba $\bar{B}$ ar Note \#14.

25. D. Williams, Notes from B Factory Particle ID Working Group, March 9, 1990, SLAC; see also, B. Ratcliff, in $B a \bar{B}$ ar Note \#30.

26. P. Coyle, Notes from B Factory Particle ID Working Group, April 20, 1990 (Nevis meeting).

27. See also, plenary talk on Particle ID, B. Ratcliff, in $B a \bar{B} a r$ Note \#37.

28. See, for example, K. Abe, et al, SLAC-PUB-5214.

29. J. Va'yra, SLAC-PUB-5207 and SLAC-PUB4116.

30. J. Va'vra, Notes from B Factory Particle ID Working Group, April 20, 1990, (Nevis meeting).

31. B. Ratcliff, Notes from B Factory Particit ID Wolking Group, April 20, 1990 (Nevis meeting).

32. J. Va'vra, Noles from B Factory Particle ID Working Group, June 6, 1990, SLAC. 
33. See also, plenary talk on Particle ID, B. Ratcliff, in $B a \bar{B}$ ar Note \#44.

34. B. Ratcliff, Notes from B Factory Particle ID Working Group, June 6, 1990, SLAC.

35. R. Arnold, et al., Design Proposal for a B Factory Detector at PSI.

36. J. Seguinot, Proceedings of the Symposium of Particle Identification at High Luminosity Hadron Colliders, April 1989, Fermilab, Batavia, IL; T. Ypsilantis, ibid.

37. P. Coyle, Working Group on Particle ID at the Workshop on Physics and Detector Issues for a High Lumirosity Asymmetric B Factory, June 6, 1990, SLAC.

38. E. Lorenz, MPI-PAE/Exp. El. 184, preprint, (1987); R. A. Eirhler, et ai., Proposal for a $B$ Meson Factory at PSI, PSI-PR-88, 1988.

39. S. S. Kistler, Nature 127, 741 (1931); S. S. Kistler, J. Phys. Chem. 34, 52 (1932).

40. J. Fricke, erogels, Springer Proceedings in Physics 6. (Springer Verlag, Berlin).

41. T. M. Tillotson, L. W. Hrubesh and I. M. Thomas, Mat. Res. Soc. Symp. Proc. 121, 685 (1988); T. M. Tillotson and L. W. Hrubesh, UCRL-Ext. Abs. 102517, preprint (1990); L. W. Hrubesh, T. M. Tillotson and J. F. Poco, UCRL-Ext. Abs. 102518 preprint, LLL (1990).

42. L. W. Hrubesh, private communication.

43. L. W. Hrubesh, UCRL-53794 preprint, LLL (1987); L. W. Hrubesh and C. T. Alviso, Mat. Res. Soc. Symp. Proc. 121, 703 (1988).

44. S. Schindler, private communication.

45. I. Holl, E. Lorenz and G. Mageras, MPI-PAE/Exp. El. 224, preprint (1990).

46. W. J. Willis and V. Radeka, Nucl. Instr. Meth. 120, 221 (1974); V. Radeka and E. Rescia, Nucl. Instr. Meth. A265, 228 (1988). 
47. We have set the coefficients for Rayleigh scattering and for absorption to $a=1.6 \times 10^{-18} \mathrm{~cm}^{3}$ and $b=1.6 \times 10^{-11} \mathrm{~cm}$, which correspond to an average scattering length $\Lambda_{s}=2.3 \mathrm{~cm}$ and an average absorption length $\Lambda_{a}=100 \mathrm{~cm}$ in the $300-600 \mathrm{~nm}$ wavelength region. The reflectivity of Kodak paint has been used (see Section 7.5).

48. H. P. Lüttenberg, Bonn-IR-88-51 preprint, Bonn Ph.D. thesis (unpublished) (1989).

49. A. Vorobiov, private communication.

50. E. Lorenz, private communication.

51. Seybold and Wagenblast, Dyes and Pigments 11, 303 (1989).

52. C. Ambrosio, et al, CERN-PPE-90.6, preprint (1990).

53. K. Yamameto, private communication.

54. K. Yamamoto et al., Nucl. Instr. Meth. A253, 542 (1987).

55. A. Weinstein, private communication.

56. The Physics Program of a High Luminosity Asymmetric B Factory, SLAC-353 (1989). 